\title{
Limits of training and testing in horses
}

Citation for published version (APA):

Bruin, G. (1996). Limits of training and testing in horses. [Doctoral Thesis, Maastricht University]. Universiteit Maastricht. https://doi.org/10.26481/dis.19961128gb

Document status and date:

Published: 01/01/1996

DOI:

10.26481/dis.19961128gb

Document Version:

Publisher's PDF, also known as Version of record

\section{Please check the document version of this publication:}

- A submitted manuscript is the version of the article upon submission and before peer-review. There can be important differences between the submitted version and the official published version of record.

People interested in the research are advised to contact the author for the final version of the publication, or visit the DOI to the publisher's website.

- The final author version and the galley proof are versions of the publication after peer review.

- The final published version features the final layout of the paper including the volume, issue and page numbers.

Link to publication

\footnotetext{
General rights rights.

- You may freely distribute the URL identifying the publication in the public portal. please follow below link for the End User Agreement:

www.umlib.nl/taverne-license

Take down policy

If you believe that this document breaches copyright please contact us at:

repository@maastrichtuniversity.nl

providing details and we will investigate your claim.
}

Copyright and moral rights for the publications made accessible in the public portal are retained by the authors and/or other copyright owners and it is a condition of accessing publications that users recognise and abide by the legal requirements associated with these

- Users may download and print one copy of any publication from the public portal for the purpose of private study or research.

- You may not further distribute the material or use it for any profit-making activity or commercial gain

If the publication is distributed under the terms of Article $25 \mathrm{fa}$ of the Dutch Copyright Act, indicated by the "Taverne" license above, 


\section{LIMITS OF TRAINING AND TESTING IN HORSES}

GRENZEN BIJ HET TRAINEN EN TESTEN VAN PAARDEN 
Bruin, Gerrit

Limits of training and testing in horses / Gerrit Bruin

Proefschrift Universiteit Maastricht. -

Met lit. opg. - Met samenvatting in het Nederlands

ISBN 90-9010063-6

Trefw.: training/ paarden

Druk: Drukkerij Cabri, Lelystad

Omslag: B.K. Bruin 


\title{
LIMITS OF TRAINING AND TESTING IN HORSES
}

\author{
PROEFSCHRIFT
}

ter verkrijging van de graad van doctor aan de Universiteit Maastricht, op gezag van de Rector Magnificus, Prof. mr. M.J. Cohen, volgens het besluit van het College van Dekanen in het openbaar te verdedigen op donderdag 28 november 1996 om 14.00 uur door

\section{Gerrit Bruin}

geboren te Vledder op 7 januari 1944 


\section{Promotores:}

Prof. Dr. H. Kuipers

Prof. Dr. G.J. van der Vusse

Beoordelingscommissie:

Prof. Dr. ir. W.H.M. Saris (voorzitter)

Prof. Dr. A. Huson (Technische Universiteit Eindhoven)

Dr. H.A. Keizer

Prof. Dr. R.J. Rose (University of Sydney, Australia)

Dr. F.T.J. Verstappen 
Voor mijn ouders, die me steeds hebben voorgehouden het stellen van vragen te verkiezen boven het geven van antwoorden 



\section{CONTENTS}

CHAPTER 1 Introduction and aim of the study

CHAPTER 2 Physiological and biochemical backgrounds of training in horses

CHAPTER 3 Variability in fiber type composition and in the content of phosphocreatine, ATP, NAD and glycogen in equine gluteus medius muscle

CHAPTER 4 Direct measurements and indirect estimation of maximal heart rate in exercising horses

CHAPTER 5 Adaptation and overtraining in horses subjected to increasing training loads: in search for early markers of overtraining

CHAPTER 6 Effects of 3-min submaximal exercise bouts combined with

endurance training on the performance capacity of horses

CHAPTER 7 Effects of $1-\mathrm{min}$ anaerobic exercise bouts, added to aerobic interval training of different intensity and duration, on the performance capacity of horses

CHAPTER 8 Effects of three and six 1-min anaerobic exercise bouts, added to continuous aerobic training, on the performance capacity of horses

CHAPTER 9 General discussion

SAMENVATIING 


\section{CHAPTER 1}

INTRODUCTION AND AIM OF THE STUDY 


\subsection{THE HORSE AS ATHLETE}

Since its domestication, the horse plays an important role in human society. The capacity to bear heavy loads at relatively high speeds makes the horse particularly apt for assisting and supporting human activities. Until the end of the last century the horse played a key role in public transportation. Nowadays in the Western world the horse is mainly used for recreational and sportive purposes. Pulling, riding (jumping, dressage, hunting) and running at high speed are the most outstanding athletic qualities, which gave the horse its popularity. One of its specific qualities is endurance capacity which can be defined as the ability to maintain relatively high speeds for a prolonged time. This ability makes the horse excellently fit for races over shorter and longer distances. Endurance capacity is mainly determined by the maximal amount of oxygen that can be taken up and delivered to the tissues. Therefore a high maximal oxygen consumption $\left(\mathrm{VO}_{2 \max }\right)$ is an important prerequisite for endurance performances. $\mathrm{VO}_{2 \max }$ is mainly determined by the capacity of the cardiorespiratory system.

The cardiorespiratory system of the horse has a high capacity compared with other species including humans. The heart weight/body weight ratio of the horse is -2 times higher than in humans, respectively 8.6 and $4.0 \mathrm{~g} / \mathrm{kg}(10)$. A determining functional feature of the heart is the amount of blood that can be pumped per unit of time (cardiac output). The cardiac output can be enhanced by increasing the heart rate and stroke volume. Both increase during exercise with increasing intensity. Compared to the resting state the horse is able to enhance cardiac output during maximal exercise eight- to ten-fold, and the arteriovenous oxygen difference five-fold (Table 1.1). The amount of blood that is pumped through the body determines the amount of oxygen that can be delivered to the tissues. Under normal resting conditions the red blood cells can bind approximately $200 \mathrm{ml} \mathrm{O}_{2}$ per litre of blood. The amount of circulating red blood cells can be increased by squeezing the spleen. This may result in an 
Chapter 1

elevation of the hematocrit (percentage of red cells per litre of blood) of more than $50 \%$. During maximal exercise, the oxygen uptake of a horse increases per kilogram body weight forty- to fifty-fold. This relative increase in oxygen uptake per kilogram body weight in the horse is twice as much as is seen in humans during maximal exertion $(11,12)$.

Table 1.1 Some physiological parameters in humans and horses at rest and at maximal exercise $^{t}$

\begin{tabular}{l|ll|ll}
\hline & \multicolumn{2}{|l|}{ Human $(75 \mathrm{~kg})$} & \multicolumn{2}{l}{ Horse $(450 \mathrm{~kg})$} \\
\cline { 2 - 5 } & Rest & Exercise & Rest & Exercise \\
\hline Heart Rate (beats $/ \mathrm{min})$ & 50 & 195 & 32 & 240 \\
Stroke Volume $(\mathrm{ml})$ & 100 & 180 & 800 & 1400 \\
Cardiac Output $(\mathrm{I} / \mathrm{min})$ & 5 & 35 & 25 & 335 \\
a-vO & 45 & 170 & 45 & 250 \\
Hematocrit $(\%)$ & 42 & 46 & 35 & 65 \\
Oxygen uptake $(\mathrm{ml} / \mathrm{kg} / \mathrm{min})$ & 2.5 & 80 & 2.5 & 160 \\
\hline
\end{tabular}

for references see $1,2,3,4,5,7$.

\subsection{TRAINING AND OVERTRAINING}

\subsubsection{Types of training}

In competitive sport it is tried to bring the performance capacity to its full potential. For this purpose the individual has to follow a training program. Training can be defined as regular exercise with a gradual increase in volume (total amount of delivered work) and intensity (speed). In general, a training program consists of repeated exercise sessions with a gradually increasing duration and/or intensity. 
Training makes use of the ability of the body to adjust to changes in external loads. Each training session leads to a disturbance of the homeostasis in tissues, organs, and organ systems. The disturbed homeostasis is associated with fatigue. In the recovery phase the body starts to restore the homeostasis, but several of the recovery processes do not stop when the original homeostasis is reached but continue for some time, leading to overcompensation. Although the mechanism underlying recovery and overcompensation is incompletely understood, these phenomena result in an enhanced functional capacity $(1,7)$. A well designed training program consists of exercise which is gradually increased in volume and intensity and matches the gradual increase in physical exercise capacity. The magnitude of the disturbances in homeostasis should be in balance with the duration of the recovery period.

The physiological response of training depends on the type, intensity and duration of exercise. A general rule for obtaining optimal sport performance is that the type of training should be as specific as possible. In general, improvement of glycolytic capacity can only be attained by high intensity, anaerobic exercise, while improvement of the capacity to maintain relatively low speeds up to several hours, can be attained most effectively by endurance training. Consequently, for enhancing the capacity of anaerobic energy metabolism near maximal sprint intensity exercise of short duration is required. When increase of aerobic capacity is strived for, the intensity of the exercise can be lower, approximating the $\mathrm{VO}_{2 \max }$ speed. In both cases the exercise intensity is close to or above the speed at which $\mathrm{VO}_{2 \max }$ is reached. For this purpose in practical training in general repeated bouts of short duration is employed, often referred to as interval training.

In interval training a subsequent exercise bout is performed before complete recovery of the previous bout is attained. The specific response to interval training may differ by varying the intensity and duration of each exercise bout as well as varying the length of the recovery period and also the number of repetitions. In interval training 


\section{Chapter 1}

the duration of each exercise bout, depending on the exercise intensity, may vary from $10 \mathrm{~s}$ to $5 \mathrm{~min}$, with recovery periods also varying from $10 \mathrm{~s}$ to $5 \mathrm{~min}$.

When interval training is employed for increasing anaerobic capacity, the intensity should be above the speed at which $\mathrm{VO}_{2 \max }$ is reached. The duration of each bout varies in practice between $30 \mathrm{~s}$ and $1 \mathrm{~min}$. Generally no more than 6 repetitions can be performed, because of increasing fatigue. The recovery between the exercise bouts varies between 1 to $4 \mathrm{~min}(1,7)$.

For enhancing aerobic capacity also interval training is used. For this purpose an intensity close to the $\mathrm{VO}_{2 \max }$ speed is chosen. In practical training of athletes, exercise bouts of $3-5 \mathrm{~min}$ at an exercise intensity of $70-90 \%$ of maximal heart rate often are used. In human athletes such an aerobic interval training session often consists of 3-6 repeated work bouts (7). The number of repetitions is usually limited by fatigue. With shorter duration of the exercise bouts, for instance $1 \mathrm{~min}$, a higher number of repetitions (10-20) can be attained. In horse training this latter type of interval training is not often used.

The other main type of training is aerobic or endurance training, consisting of continuous exercise at an intensity which may vary between $50 \%$ and approximately $90 \%$ of the maximal oxygen consumption $\left(\mathrm{VO}_{2 \max }\right)$. Because the relationship between oxygen consumption and heart rate is linear, heart rate is generally used to quantify the intensity of endurance exercise. Because the exercise intensity is below the speed at which $\mathrm{VO}_{2 \max }$ can be reached a limited improvement of $\mathrm{VO}_{2 \max }$ can be expected by endurance training. In humans it has been shown that for improving $\mathrm{VO}_{2 \max }$ in relatively untrained subjects the exercise intensity should be $70-90 \%$ of maximal heart rate, and that an intensity of $50 \% \mathrm{VO}_{\max }$ is insufficient to increase $\mathrm{VO}_{2 \max }$.

Although in humans different types of training have been studied by several investigators (7), in horses little is known about the physiological response to the different types of interval training and endurance training. The most difficult, but also most important issue of training is to find the optimal intensity and volume for each individual, leading to the best results in competition. Below this optimal level, 
performance most probably fails to reach its maximum, while a too strenuous training program can lead to overtraining.

\subsubsection{Overtraining, overreaching and staleness: occurrence and definitions}

If the duration of the recovery is insufficient to restore the exercise-induced disturbance in homeostasis, recovery is incomplete and premature fatigue may be encountered. One may speak of overtraining. The term overtraining is used in all instances where recovery is incomplete and the training load exceeds the functional capacity of organs or organ systems. If the imbalance between exercise-induced disturbance in homeostasis and recovery is continued for some days, one may speak of short-term overtraining or overreaching. Short term overtraining is usually characterised by increase of fatigability. Overreaching is generally reversible within a couple of days, if the daily training load is lowered. When the imbalance between exercise and recovery is maintained for several days, in addition of increased fatigability also behavioural symptoms are often seen. This advanced overtraining is referred to as overtraining syndrome or staleness (6). In staleness a complex of symptoms may be observed such as irritability, diminished food intake and a lowered performance capacity (6). Overreaching and staleness are frequently found in horses as well as in man. The symptoms of overreaching and staleness are in general similar in both species $(8,9)$.

\subsubsection{Practical aspects of horse training}

In human sport, training is to a certain extent based on the results of experimental research in exercise physiology. In general, this is not the case in equine sport. In Thoroughbreds and standard bred trotters and pacers the ultimate goal is running with the highest possible speed over distances ranging from $1000 \mathrm{~m}$ to 7000 $\mathrm{m}$, but mostly shorter than $2500 \mathrm{~m}$. A high maximal oxygen uptake is a prerequisite for these types of performances. The training program generally consists of a mixture of 
Chapter 1

endurance running at intensities below $\mathrm{VO} 2_{\max }$ and interval training close to or above the $\mathrm{VO}_{2 \max }$ speed. The exercise intensity, duration of each exercise bout and the number of repetitions is mostly based on experience and intuition rather than on the outcome of scientific studies. Often the different factors contributing to actual racing performance, such as acceleration, anaerobic power, and $\mathrm{VO}_{2 \max }$ are rarely specifically trained. So, in the horse the precise physiological responses to different types, intensities and volumes of training remain largely unknown.

\subsection{AIMS OF THE PRESENT STUDY}

Although a host of training studies have been conducted in man, relatively little is known about the physiological response to various types of training in the horse. Although interval training and endurance training are employed in equine sport, the applied exercise intensity and the number of repetitions have little scientific basis. To conduct well controlled studies in practice is very difficult because the external conditions make standardisation virtually impossible. Not only the effects of different types of training are largely unknown, also the optimal amount of exercise is uncertain. The main reason lies in the fact that at present in horses the relationship between volume of training and physiological response, which is relevant for optimising the training and to prevent overtraining, is largely unknown.

Ta study the physiological basis of training effects one may look at blood chemical variables and biochemical variables in muscle tissue. To perform these types of analysis the reproducibility of the methods and variables used should be known. Therefore one of the aims of this thesis is to study the variability of fiber type distribution and some biochemical variables in muscle biopsies taken from the gluteus medius muscle from various spots and depths to delineate the region of choice for obtaining biopsies in further investigations (chapter 3 ).

Effects of training are generally assessed by exercise tests, which measure an overall effect. The most frequently used variables are heart rate and plasma lactate as a 
function of running speed. However, to draw conclusions about certain changes the unexplained variability of the parameters should be known. Therefore, one of the aims of this thesis is to study the variability of the relationship between exercise intensity, heart rate and plasma. lactate concentration. Although commercially available heart rate monitors are often used in practice, its reliability at high frequencies is poorly investigated in horses. Therefore the heart rates obtained by ECG were compared with heart rates measured by a commercially available heart rate monitor. In addition, the accuracy of two mathematical models with plasma lactate concentration and heart rate at submaximal intensity as input variables, for estimating maximal heart rate, was investigated (chapter 4).

In the present study all training and testing was controlled because all exercise was done on a treadmill. One of the purposes of this thesis is to investigate physical performance capacity and physiological adaptations to a training protocol consisting of exercise that gradually increased in volume and intensity and to seek for indicators for early (overreaching) and advanced overtraining (staleness) (chapter 5). Such an indicator would help to optimise the training process and to prevent advanced overtraining.

In humans as well as in horses, aerobic and anaerobic exercise is used to increase the physical performance capacity. For a scientific based choice of the type of exercise at different stages of the training process, more knowledge is needed about the effects of the different types of exercise. One of the aims of this thesis is to study the effects of different training programs that are employed in practice on the physical performance capacity of horses. Therefore, exercise programs with mainly aerobic exercise (chapter 6), a combination of aerobic and anaerobic exercise (chapter 7) and anaerobic exercise (chapter $\mathbf{8}$ ) have been studied. Performance was measured on the treadmill as maximal running speed, total running time and total amount of work delivered. To obtain insight into the mechanisms of the effects of the training 
Chapter 1

protocols, muscle biopsies were taken to investigate various compounds involved in energy metabolism.

In the last chapter of this thesis (chapter 9) all findings of this study are discussed in the light of literature available and the application to the practice of horse training.

\subsection{REFERENCES}

1. Åstrand P.O., K. Rodahl. Textbook of Work Physiology. Physiological bases of exercise. McGraw-Hill Book Company, New York, 1986.

2. Evans D.L., R.J. Rose. Maximum oxygen uptake in racehorses: changes with training and prediction from submaximal cardiorespiratory measurements. In: Gillespie J.R., N.E. Robinson (eds): Equine Exercise Physiology 2 Edition, Edwards Brothers, Ann Arbor, USA, 1987, pp. 52-67.

3. Fregin G.F., D.P. Thomas. Cardiovascular Response to Exercise in the Horse: A Review: In: Snow D.H., S.G.B. Persson, R.J. Rose (eds): Equine Exercise Physiology, Burlington Press, Cambridge, 1983, pp. 76-90.

4. Jones J.H. "K.E. Longworth, A. Lindholm, K.E. Conley, R.H. Karas, S.R. Kayar, C.R. Taylor. Oxygen Transport During Exercise in Large Mammals. 1. Adaptive Variation in Oxygen Demand. J Appl Physiol 67: 862-870, 1989.

5. Knight P.K., A.K. Sinha, R.J. Rose. Effects of training intensity on maximum oxygen uptake. In: Persson S.G.B., A. Lindholm, L.B. Jeffcott (eds): Equine Exercise Physiology 3, Almqvist \& Wiksell Tryckeri, Uppsala, 1991, pp. 77-82.

6. Kuipers H., H.A. Keizer. Overtraining in Elite Athletes. Review and Directions for the Future. Sports Med 6: 79-92, 1988.

7. McArdle W.D., F.I. Katch, V.L. Katch. Training for anaerobic and aerobic power. In: Exercise Physiology. Energy, nutrition and human performance, Lea \& Febiger, Philadelphia, 1986, pp. 347-370.

8. Persson S.G.B., B. Essen, A. Lindholm. Oxygen uptake, red-cell volume, and pulse/work relationhip in different states of training in trotters. Proc Fifth Meeting Acad Soc Large Animal Vet Med, Glasgow: 34-43, 1980. 
9. Persson S.G.B.. Evaluation of Exercise Tolerance and Fitness in the Performance Horse. In: Snow D.H., S.G.B. Persson, R.J. Rose (eds): Equine Exercise Physiology, Burlington Press, Cambridge, 1983, pp. 441-457.

10. Snow D.H.. The horse and dog, elite athletes- why and how? Proc Nutrit Soc 44: 267-272, 1985.

11. Thomas D.O., G.F. Fregin. Cardiorespiratory and metabolic responses to treadmill exercise in the horse. J Appl Physiol: Resp Environ Exercise Physiol 50: 864-868, 1981.

12. Weber J.M., G.P. Dobson, W.S. Parkhouse, D. Wheeldon, J.C. Harman, D.H. Snow, P.W. Hochachka. Cardiac output and oxygen consumption in exercising Thoroughbred horses. Am J Physiol 253: R890-R895, 1987. 
Chapter 2

\section{PHYSIOLOGICAL AND BIOCHEMICAL BACKGROUNDS OF TRAINING IN HORSES}

This chapter will be published as:

G. Bruin, H. Kuipers and G.J. van der Vusse

Physiological and biochemical backgrounds of training in horses

Int J Sports Med (Accepted for publication) 
Chapter 2 


\subsection{INTRODUCTION}

Since an important part of the present study was focused on muscle tissue and its energy metabolism, a brief overview of the main features of skeletal muscle physiology and energy metabolism is presented.

\subsection{SKELETAL MUSCLE}

Skeletal muscle tissue is formed in the embryonic phase during which myoblasts, which will eventually form skeletal muscle, fuse and form tubes, the myofibers. After fusion to myofibers the nuclei migrate to the periphery while the core of the muscle fiber is filled with organelles, directly or indirectly involved in contractile activity.

The functional unit of a muscle consists of the motor-unit, a group of muscle fibers innervated by one single motoneuron. Each muscle fiber contains a number of organelles, of which the myofibrils occupy the largest volume and are responsible for the contractile process. Each myofibril consists of actin and myosin filaments. The myosin filament is composed of myosin molecules, each consisting of two heavy chains and four light chains. One end of the two heavy chains is folded into a globular protein, the myosin head. This part of the myofibril contains myosin-ATPase. The actin filament consists of three different proteins: actin, tropomyosin and troponin. Troponin is composed of three different protein subunits with respectively a strong affinity for actin, tropomyosin and $\mathrm{Ca}^{2+}(1,22,59)$. During muscle contraction the myosin head binds with actin and actomyosin is formed. The troponin-tropomyosin complex inhibits the myosin-actin binding. During depolarisation small quantities of $\mathrm{Ca}^{2+}$-ions are released from the sarcoplasmic reticulum into the cytosol. A proportion of these $\mathrm{Ca}^{2+}$-ions binds to troponin. The troponin supposedly undergoes a structural change that results in a movement of the tropomyosin. This "uncovers" the active sites of the actin, thus allowing contraction to initiate and proceed. To allow relaxation $\mathrm{Ca}^{2+}$-ions 


\section{Chapter 2}

are transported back into the sarcoplasmic reticulum by an ATP-driven pump resulting in a disconnection of actin and myosin.

Based on myosin ATP-ase activity which is related to the speed of contraction, muscle fiber types can be divided histochemically into two main classes, slow-twitch (type I) and fast-twitch (type II) fibers (Fig. 2.1).

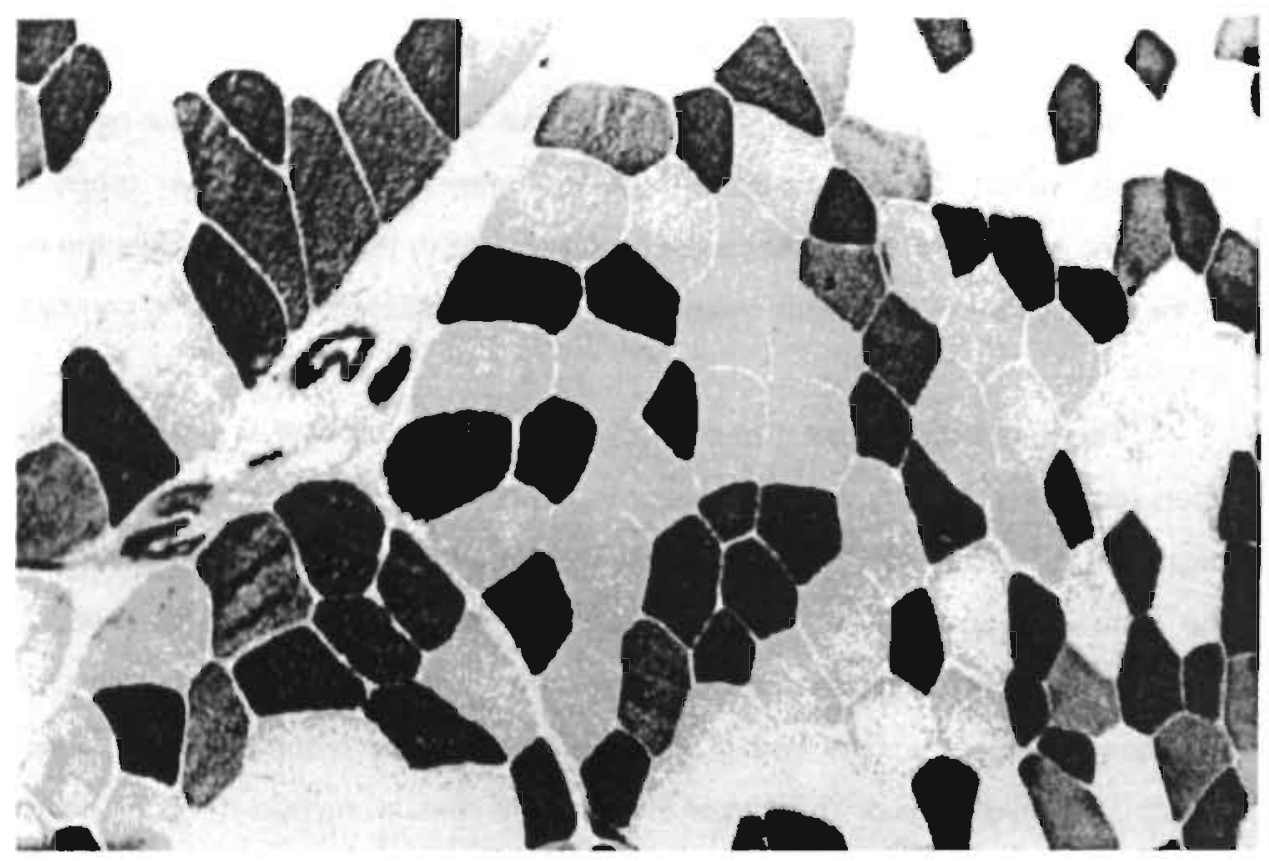

Fig. 2.1 Cross section of human quadriceps femoris muscle stained with myofibrillar ATP-ase, pre-incubated at $\mathrm{pH}=4.6$. The dark fibers represent type I, the lightly stained type $I / b$, and the unstained fibers type Ila.

Making use of different $\mathrm{pH}$-labilities of the various isoforms of myosin ATP-ase, the type II population can be further subdivided into type IIa, IIb, and type IIc. In general type Ila has a higher oxidative capacity than type IIb, while type lic fibers are considered as young, undifferentiated fibers (32). It is generally assumed that the fiber type distribution in the different muscles is genetically determined so that after birth fiber-type distribution changes only minimally $(13,25,51)$. 
In general, type I (slow twitch) fibers have a higher oxidative capacity than type II fast twitch fibers. Therefore type I fibers have a higher fatigue resistance than type II fibers. Although a correlation between contractile properties and oxidative capacity is generally found, in humans as well as in horses a large variation in oxidative capacity is seen within muscle fibers belonging to the same type (64).

Each skeletal muscle contains a mixture of fibers belonging to the type I and the type II population. Between horse breeds there are large differences in fiber type distribution. The horses bred for speed have a larger percentage type II fibers. In Standard bred trotters there are about $75-80 \%$ type 11 fibers in the gluteus medius muscle $(37,56)$. The presence of different fiber types in each muscle has an important functional significance, because the motor neurons innervating the different fiber types differ in recruitment threshold. During low-intensity exercise mainly type I fibers are activated (41). In exercise of longer duration and/or higher intensity not only type I but also a larger number of type II fibers are recruited, while at the highest intensities all fiber types are active at the same time $(12,19,21,24,55,65)$.

For studying metabolic processes in skeletal muscle, the needle biopsy technique is a convenient and generally employed method to take small tissue samples (50-200 mg) from muscular tissue (3). Because in humans the quadriceps femoris and the gastrocnemius muscles are involved in a variety of physical activities, these muscles are often used for biopsy sampling. In horses the gluteus medius muscle is important in running $(36,57)$. Therefore in horses this muscle is commonly used for taking muscle biopsies.

\subsection{ENERGY METABOLISM}

Muscle contraction requires a continuous supply of energy in the form of ATP. Hydrolysis of this high energy phosphate compound yields energy to allow for proper functioning of the actin-myosin complex and intracellular ion pumps. ATP is regenerated by aerobic and anaerobic metabolic processes. Substrates for aerobic 


\section{Chapter 2}

energy conversion are glucose, fatty acids, ketone bodies and to a minor extent amino acids. Anaerobic regeneration of ATP is mainly achieved by phosphocreatine and by substrate-linked ATP formation in the glycolytic pathway with glucose as substrate. Glucose can be derived from extracellular sources or from the endogenous carbohydrates store, i.e., glycogen.

In figure 2.2 a very simplified scheme of muscle energy conversion is presented, indicating the glycolytic pathway (the conversion of glucose into pyruvate), the incorporation of glucose in the intracellular carbohydrate store, and the conversion of pyruvate into lactate (anaerobic conditions) or into acetyl CoA (aerobic conditions). Moreover , the incorporation of fatty acids in the intracellular lipid pool (triacylglycerols) and the stepwise degradation of fatty acids into acetyl CoA via the B-oxidation pathway are shown. Ketone bodies and some amino acids may also serve as substrates for the formation of acetyl CoA. Acetyl CoA plays a central role in muscle aerobic energy conversion as it provides carbon and hydrogen atoms to the citric acid cycle.

Condensation of the acetyl residue of acetyl CoA with oxaloacetate yields citric acid. The latter compound is stepwise degraded to oxaloacetate. Energy stored in citric acid is used to regenerate ATP from ADP and inorganic phosphate in a highly controlled system, the so-called respiratory chain. The availability of molecular oxygen is an absolute prerequisite for respiratory chain-linked ATP formation. Below, the uptake and intracellular conversion of glucose and fatty acid, and muscle high-energy phosphate metabolism will be discussed in more detail. For detailed information of the citric acid cycle and the function of the respiratory chain the reader is referred to general textbooks of biochemistry $(10,59)$. 


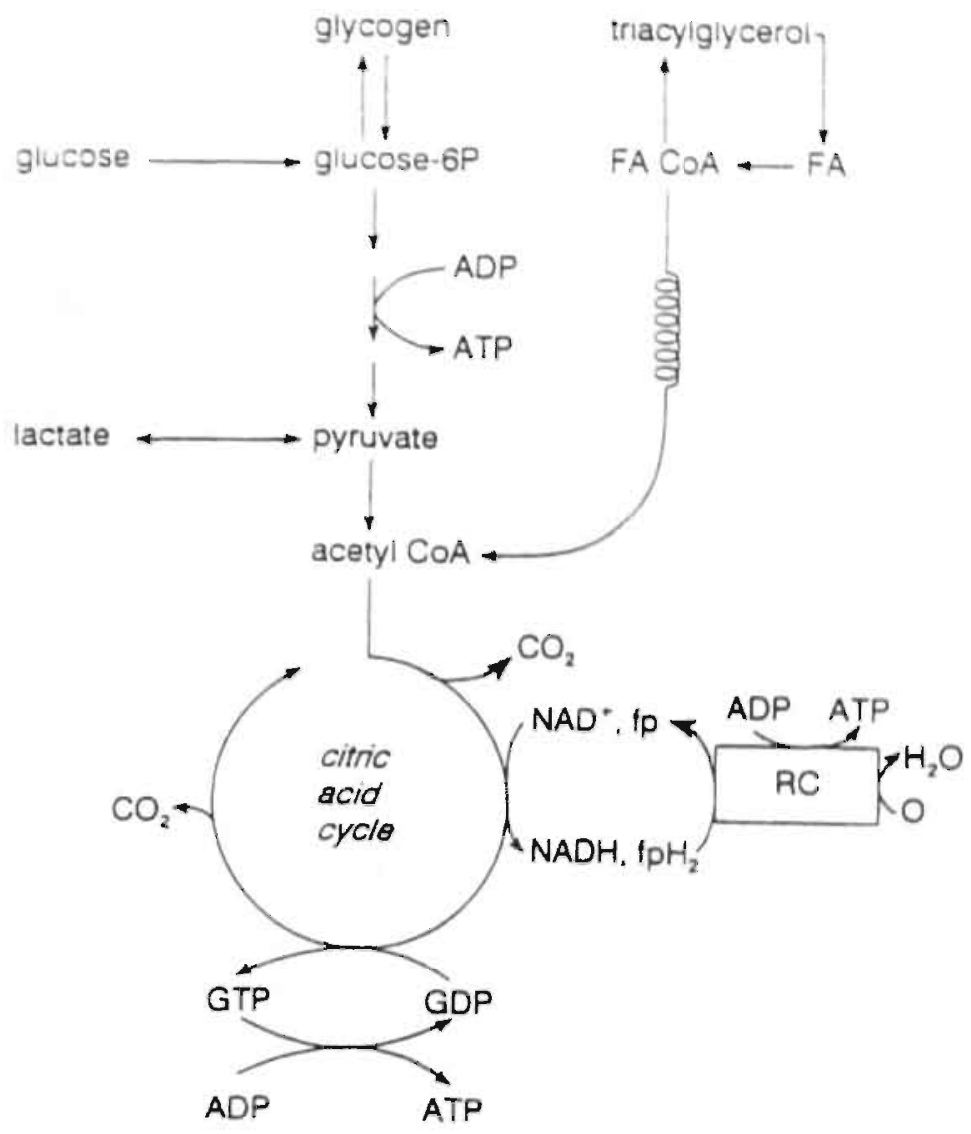

Fig. 2.2 Highly schematic representation of muscular energy converting pathways. FA refers to fatty acids, RC to respiratory chain, fp to flavoprotein, GDP and GTP to guanosine diphosphate and triphosphate, respectively, and $\mathrm{O}$ to molecular oxygen. 


\subsubsection{Uptake and conversion of carbohydrates in skeletal muscle cells.}

Glucose is the main carbohydrate that serves as substrate for muscular ATP production. Either derived from diet or synthesised in the liver, glucose is transported via the bloodstream to muscle cells. The bulk of glucose diffuses through endothelial clefts to the interstitial compartment. Transport of glucose from the interstitium to the cytoplasm across the sarcolemma is protein-mediated (Fig. 2.3). Recent investigations have revealed the existence of a variety of glucose transporting proteins (48). At least two members of the family of glucose transporters (i.e., GLUT1 and GLUT4) have been identified in muscles. Evidence is accumulating that the biological activity of GLUT4 is regulated by insulin. Insulin promotes the transition of GLUT4 from intracellular stores to the sarcolemma. Less information is present on the regulation of the biological activity of GLUT1 in skeletal myocytes.

After extraction from the interstitial compartment glucose is rapidly converted to glucose 6-phosphate by hexokinase. Phosphorylation of the glucose moiety most likely prevents back-diffusion (or transport) of the glucose moiety to the extracellular space. Under resting conditions the bulk of glucose is used to replenish the glycogen stores exhausted during previous bouts of exercise. To this end glucose 6-phosphate is converted to glucose 1-phosphate, and subsequently incorporated via UDP-glucose in glycogen. The latter process is catalysed by glycogen synthase. Release of glucose from the intracellular carbohydrate pool is achieved by action of glycogen phosphorylase, yielding glucose 1-phosphate.

Both glycogen synthase and glycogen phosphorylase are highly controlled enzymes. Phosphorylation of the enzymes as occurs after stimulation of the muscle cells by agonists like (nor)adrenaline, results in inactivation of glycogen synthase and activation of glycogen phosphorylase. $\mathrm{Ca}^{2+}$ ions, the concentration of which increases during muscular activity, also promote activation of glycogen phosphorylase and inhibition of glycogen synthase via alterations of the degree of phosphorylation of the two enzymes. 
Fig. 2.3 (For legend see next page)

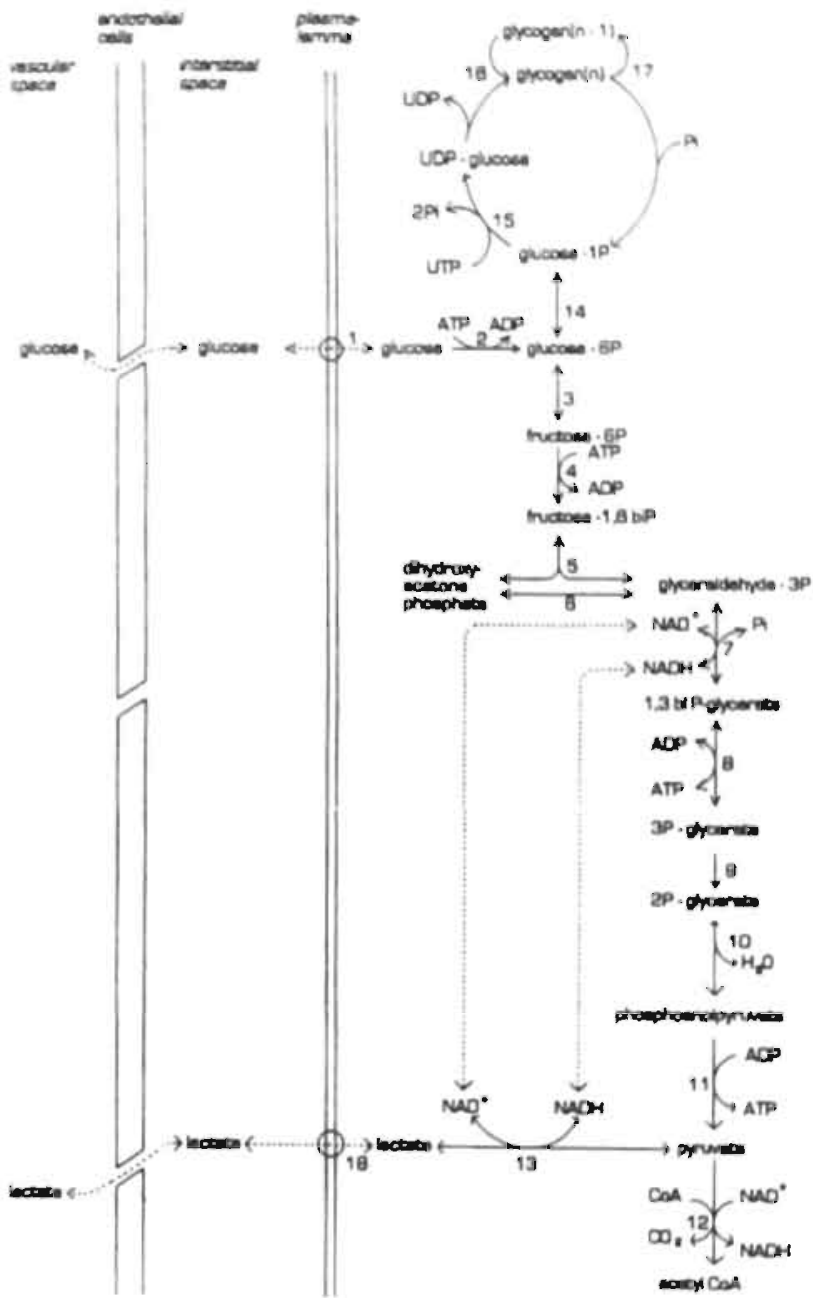


Fig. 2.3 Schematic overview of cellular carbohydrate metabolism. Arrows with solid and broken lines refer to metabolic conversions and routes of transport, respectively. Pi refers to inorganic phosphate, UDP to uridine diphosphate, and UTP to uridine triphosphate. Number 1 through 18 refer to proteins or enzymes catalyzing the intracellular conversion of carbohydrates; 1 , glucose transporter (GLUT); 2, hexokinase; 3, phosphoglucose isomerase; 4, 6-phosphofructose1-kinase; 5, fructose-biphosphate aldolase; 6 , triose phosphate isomerase; 7 , glyceraldehyde-3P dehydrogenase; 8, phosphoglycerate kinase; 9, phosphoglycerate mutase; 10, enolase; 11 , pyruvate kinase: 12, pyruvate dehydrogenase; 13, lactate dehydrogenase; 14, phosphoglucomutase; 15, glucose-1P' uridylyltransferase; 16, glycogen synthase (and 1,4-aglucan branching enzyme); 17, glycogen phosphorylase (and debranching enzyme); 18, lactate transporter.

Moreover, other humoral factors such as AMP, ATP, and glucose 6-phosphate are exerting a regulatory effect on the rate of glycogen synthesis and degradation.

Glucose 1-phosphate is stepwise degraded to pyruvate in the glycolytic pathway (Fig. 2.3). A crucial step in the glycolytic pathway is the conversion of fructose 6phosphate to fructose 1,6-biphosphate catalysed by 6-phosphofructo-1-kinase. The activity of this enzyme is regulated by a variety of factors. In general, the enzyme is active during moderate muscle activity and inactive under resting conditions. AMP, fructose 1,6-biphosphate and inorganic phosphate exert a stimulating allosteric effect on the enzyme, whereas ATP, citrate and hydrogen ions (low pH) have a negative effect on the activity of 6 -phosphofructo-1-kinase. In the contracting muscle fibers, the intracellular levels of AMP, inorganic phosphate and fructose 1,6-biphosphate are increased resulting in an enhanced glycolytic flux. During rest, ATP levels are high which will impede the flux through the glycolytic pathway. Inhibition of 6-phosphofructo-1-kinase by citrate can occur both in resting and contracting muscle fibers and is thought to play a role in the competition between fatty acids and glucose in oxidative energy conversion, since increased cellular consumption of fatty acids is known to be accompanied with elevated citrate levels. The inhibition of the enzyme 
and, hence, glycolytic flux by hydrogen ions is most likely a safety measure of the muscle cell to slow down the glycolytic production of lactate under anaerobic conditions in order to prevent an excessive drop in intracellular $\mathrm{pH}$, which may lead to damage of cellular structures.

Fructose 1,6-biphosphate is cleaved into two 3-carbon containing glycolytic intermediates, i.e., dihydroxyacetone phosphate and glyceraldehyde 3-phosphate by action of the enzyme aldolase. The 3-carbon intermediates are subsequently converted to pyruvate. The latter substance is produced from phosphoenol pyruvate by pyruvate kinase. The activity of pyruvate kinase is controlled by phosphorylation of the enzyme. In anaerobic conditions NADH produced is utilised to convert pyruvate into lactate. It is of interest to note that in the first stage of the glycolytic pathway 2 moles of ATP are invested, whereas in the trajectory from glyceraldehyde 3-phosphate to pyruvate 4 moles of ATP are produced. Hence, the net production of ATP in this metabolic pathway is 2 moles of ATP under anaerobic circumstances. In case glucose is aerobically degraded via the citric acid cycle to $\mathrm{CO}_{2}$ and $\mathrm{H}_{2} \mathrm{O}$ in toto 36 . or 38 moles of ATP are produced per mole of glucose.

With respect to muscle giucose metabolism substantial differences exist between type I and type II muscle fibers. In addition, species differences have to be considered. Since fast twitch fibers (type II) rely more on glycolytic ATP production than oxidative slow twitch fibers (type 1), it is not unexpected that the glycolytic capacity of fast-twitch fibers exceeds that of slow-twitch type I fibers. Moreover, the resting content of glycogen is higher in type II than in type I fibers (12). In equine muscles the amount of glycogen is in general twice as high than in corresponding muscles in men (45).

\subsubsection{Uptake and conversion of fatty acids in skeletal muscle cells}

Lipids are the main source of energy of muscles, exercising under normal, aerobic circumstances. Lipids are supplied to the muscle cells via the bloodstream 


\section{Chapter 2}

either in the form of fatty acids incorporated in the triacylglycerol core of circulating lipoproteins, or complexed to albumin (Fig. 2.4).

Fig. 2.4 (For legends see next page)

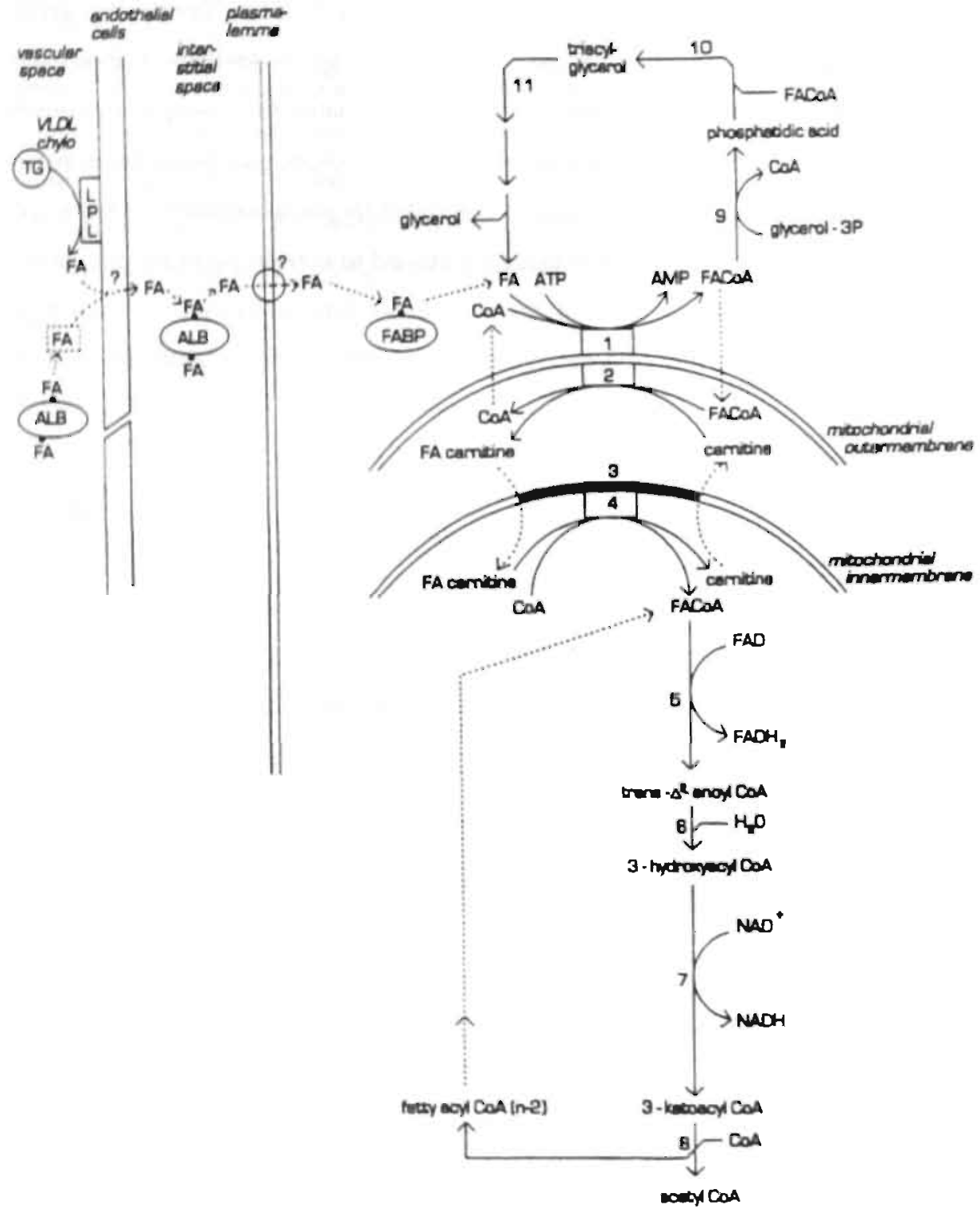


Fig. 2.4 Schematic representation of fatty acid degradation and incorporation in triacylglycerols. Solid and broken arrow lines refer to metabolic conversions and transport routes, respectively. ALB refers to albumin, VLDL to very low density lipoproteins, chylo to chylomicrons, LPL to lipoprotein lipase, TC to triacylglycerol in the core of chylomicrons and VIDL. FA to fatty acid or fatty acyl, FABP to fatty acid-binding protein, CoA to coenzyme A. Numbers refer to enzymes and transport proteins; 1, fatty acyl CoA synthetase; 2, carnitine acyltransferase (CAT-1); 3, carnitine-acylcarnitine translocase; 4, carnitine acyltransferase (CAT-II); 5, fatty acyl CoA dehydrogenase; 6, enoyl CoA hydratase; 7, 3-hydroxyacyl COA dehydrogenase; 8, 3 ketothiolase; 9, acyltransferase I and II; 10, phosphatidate phosphate and acyltransferase III; 11 . triacylglycerol, diacylglycerol and monoacylglycerol lipase.

The former source of fatty acids originates from liver (very low density lipoproteins) or from intestinal cells (chylomicrons). Fatty acids are released from the lipoproteintriacylglycerol core by lipoprotein lipase, an enzyme attached to the luminal membrane of endothelial cells, constituting the wall of the microvasculature (69).

Albumin-bound fatty acids originate from adipose tissue. These substances are released from fat cells by action of hormones such as adrenaline. Prior to uptake in muscle cells, fatty acids are dissociated from the albumin-carrier, and transported through the microvascular endothelium vial an incompletely understood mechanism (69). The same route is followed by fatty acids hydrolysed from lipoproteintriacylglycerols.

Fatty acids are subsequently transported from the interstitial space to the cytoplasm across the cellular membrane of the skeletal myocytes. Recent findings suggest that membrane proteins are facilitating transmembrane transport of (part of) fatty acids (69).

Inside the myocyte fatty acids cross the space from sarcolemma to the intracellular sites of metabolic conversion by diffusion. Fatty acid-binding protein is most likely involved in this cytosolic transport process (Fig. 2.4). Fatty acids can be converted at the endoplasmic reticulum, the peroxisomal membrane, and the mitochondrial outer 
Chapter 2

membrane. In skeletal muscle cells the bulk of fatty acids is most likely converted into fatty acyl COA by fatty acyl COA synthetase located at the mitochondrial outer membrane. The two other sites of conversion play a minor role in this cell type (69).

Fatty acyl CoA stands at a crossroad of various metabolic pathways (Fig. 2.4). Part of the acyl residues of this compound is incorporated in the endogenous triacylglycerol-pool and in membrane phospholipids. In exercising muscles the bulk of fatty acids extracted from the extracellular environment is further metabolised in the mitochondrial matrix. To reach this space the fatty acyl residue of acyl CoA has to be transported across the mitochondrial inner membrane. As the inner membrane is virtually impermeable for acyl CoA, acyl CoA is first converted to fatty acylcarnitine by carnitine acyltransferase I. Acylcarnitine crosses the inner membrane in exchange of a molecule carnitine. Inside the mitochondria fatty acylcarnitine is converted to fatty acyl CoA, and stepwise degraded to acetyl CoA in the B-oxidative pathway (Fig. 2.4).

The acetyl residue of acetyl CoA is subsequently oxidised to $\mathrm{CO}_{2}$ and $\mathrm{H}_{2} \mathrm{O}$ via the citric acid cycle and respiratory chain, both located in the matrix and inner membrane of the mitochondria. The total amount of ATP produced by complete oxidation of a fatty acid depends on the number of carbon-atoms and unsaturated bonds in the aliphatic chain of the fatty acid. For instance, complete oxidation of one mole of palmitic acid (C 16:0) yields 129 moles of ATP.

In acidition to fatty acids derived from extracellular sources, fatty acids present in triacylglycerols in cytoplasmic lipid droplets can readily serve as oxidizable substrates. Various types of triacylglycerol lipases are present to liberate fatty acids from the endogenous lipid store. It is nowadays. generally accepted that the hormone-sensitive lipase plays a crucial role inn endogenous triacylglycerol hydrolyșiș.

Experimental findings indicate that in skeletal myocytes substantial differences exist between fast and slow-twitch fibers with respect to the triacylglycerol content. In humans, the oxidative, slow-twitch type I fibers contain 3 to 5 times more endogenous triacylglycerols than type II fibers. Species differences are also present. Rat and monkey skeletal muscles were found to contain less triacylglycerols than corresponding muscles 
in man and dog (69). The activity of enzymes and the content of proteins involved in cellular fatty acid handling are in general significantly higher in type I muscle cells than in fast-twitch, glycolytic type II muscle fibers (69).

\subsubsection{Alternative substances for muscle energy production}

In addition to glucose and fatty acids skeletal muscle cells are able to utilise lactate, ketone bodies and some amino acids as oxidizable substrates. During submaximal exercise the slow-twitch type I fibers extract lactate from the interstitial space, and oxidise this substrate after conversion via pyruvate to acetyl CoA. The bulk of lactate is produced by the neighbouring glycolytic type II fibers (61, 62). In submaximally exercising horses on the order of $5 \%$ of total oxygen consumption is used for oxidation of lactate (70). In the resting muscle the amount of lactate used for energy conversion is less than during exercise, and the bulk of circulating lactate is utilised for de novo synthesis of glucose in the liver. Hence it is important to realise that lactate levels in blood plasma reflect the difference between production in muscles and utilisation in heart, liver and type I muscle fibers.

Ketone bodies are produced in the liver during fasting and are released into the vascular compartment. These substrates can be easily extracted by skeletal muscle cells and oxidised in the mitochondrial matrix after conversion into acetyl CoA.

A variety of amino acids can also serve as energy donors in exercising muscle after transamination and/or deamination of the amino group. Their contribution, however, is limited in normal conditions (59).

\subsubsection{High-energy phosphates}

As pointed out above, high-energy phosphates, such as ATP, play a pivotal role in skeletal muscle contraction. The chemical energy stored in the terminal phosphate bond of ATP is used for contraction and relaxation of the actin-myosin complex. Moreover, ATP is required for maintaining the cellular ion concentrations at physiological levels via ATP-dependent membrane ion pumps. 
As discussed earlier, ATP is produced in the glycolytic, anaerobic pathway, and by oxidative phosphorylation in the mitochondrial matrix. ATP is transported across the mitochondrial inner membrane in exchange of ADP. The bulk of ATP reacts with creatine yielding phosphocreatine and ADP. This reaction is catalysed by mitochondrial creatine kinase. ADP is transported across the mitochondrial inner membrane, back into the matrix, in exchange of ATP. Phosphocreatine diffuses through the cytosol to sites in need of energy. In the cytosolic space phosphocreatine reacts with ADP giving rise to the formation of ATP and creatine. Creatine diffuses back to the mitochondrion and ATP donates its energy for muscle contraction and/or ion pump activity.

Under normal circumstances the cellular ATP content is kept at a constant level. In the absence of $\mathrm{O}_{2}$, phosphocreatine is used to replenish the ATP store. Substratelinked phosphorylation in the glycolytic pathway is also capable to regenerate ATP from ADP under oxygen-restricted conditions. These processes prevail during shortterm exercise at relatively high intensity. In submaximal exercise of a duration longer than 30 to $60 \mathrm{~s}$ oxidation of glucose and/or fatty acids in mitochondria, located between the myofibrils or close to the sarcolemma, is required to generate ATP from ADP and inorganic phosphate. Striking differences exist in mitochondrial density between slow-twitch type I and outspoken glycolytic type IIb fibers. The mitochondrial content in the latter muscle cells is considerably lower than in type I fibers. In contrast, the mitochondrial density of type Ila fibers is in the same order of magnitude of that of type I fibers. Despite this fact, it has been found in rat muscle, that the turnover rate of the cellular ATP pool is approximately 2 to 4 times higher in type lla than in type I fibers during short-term near maximum aerobic exercise (23).

When ATP utilisation exceeds the regeneration of ATP, the cellular ATP level starts to drop. In the first line of defence stands the enzyme myokinase, catalysing the reaction $2 \mathrm{ADP}=>\mathrm{ATP}+\mathrm{AMP}$ (Fig. 2.5). 
mitochovation

$A D P-A$ ADP -

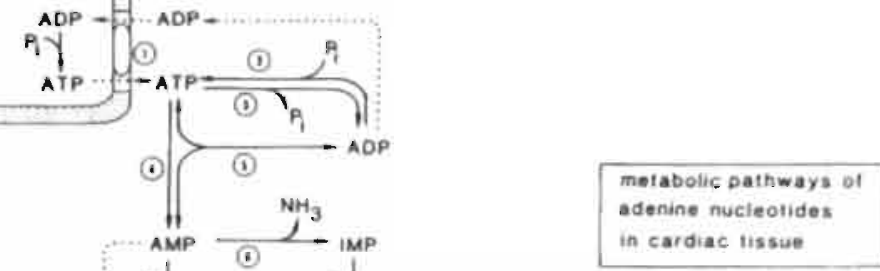

intracelluiar

space

(1) $\left.\right|_{-P} \quad N H_{3}$ (1)

adenosine $\frac{1}{6}$ inosine

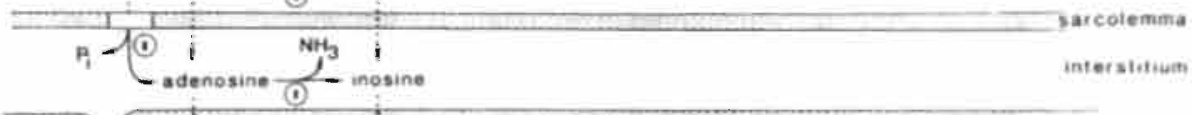

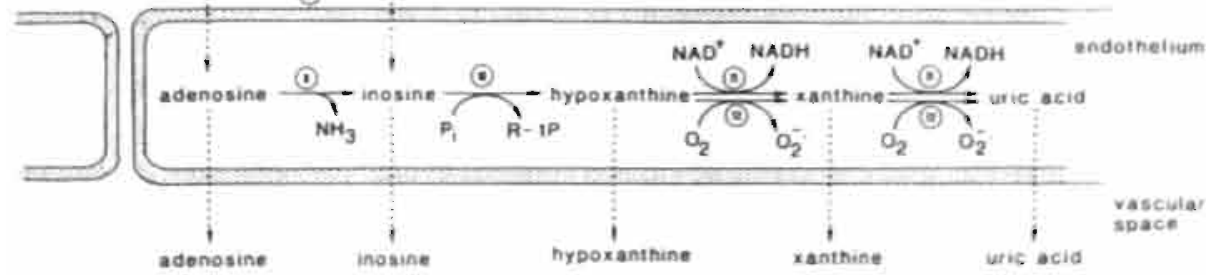


Chapter 2

Fig. 2.5 Major pathways involved in the degradation of adenine nucleotides. Dotted lines indicate transport of substances, Pi refers to inorganic phosphate, PPi to pyrophosphate, R-1P to ribose-1-phosphate, and $\mathrm{O}_{2}$ - to superoxide anion; 1, adenine nucleotide translocase; 2, glycolytic ATP production; 3, energy requiring processes; 4, pyrophosphate forming enzymes; 5 , adenylate kinase (myokinase); 6, AMP-deaminase; 7,endo-5'-nucleotidase; 8, ecto-5'-nucleotidase; 9, adenosine deaminase; 10, nucleoside phosphorylase; 11, xanthine dehydrogenase, and 12, xanthine oxidase (with permission from Van Bilsen (74)).

Although this reaction is helpful to regenerate ATP from ADP it cannot prevent a further decline in cellular ATP content. AMP starts to accumulate, which will result in the formation of IMP (a metabolic conversion catalysed by AMP deaminase). Alternatively, AMP can be catabolised to adenosine by $5^{\prime}$ nucleotidase (Fig. 2.5). The same enzyme catalyses the degradation of IMP to inosine. Removal of the amino group of adenosine by action of adenosine deaminase also results in the formation of inosine. Inosine is further degraded to hypoxantine, xanthine, and uric acid in the microvascular ondothelial colls (Fig, 2.5). The latter motabolic stops aro catalysod by inosine phosphorylase and xanthine dehydrogenase/oxidase, respectively.

Accumulation of IMP in skeletal muscular tissue has been proven to be a sensitive marker for the imbalance in ATP utilisation and regeneration (50). Production of IMP can occur in both type I and type II fibers (49). In contrast to the phosphate containing nucleotides, such as ATP, ADP, AMP and IMP, nucleosides and (oxy)purines (inosine, adenosine, hypoxine, xanthine) can easily leave the cell and are removed by blood streaming through the microvascular bed.

ATP can be resynthetised from hypoxanthine (by the salvage pathway) or de novo from phosphoribosyl pyrophosphate, glutamine and glycine. The latter anabolic process is highly energy dependent and is relatively slow (59). 


\subsection{TRAINING ADAPTATIONS}

It is important to realise that performance is determined by several organ systems and that the adaptations to training affect all organ systems in the body. A brief overview is presented of the main training effects.

\subsubsection{Training and the cardiorespiratory system}

As parameter for the aerobic capacity generally the maximal oxygen consumption $\left(\mathrm{VO}_{2 \max }\right)$ is used. Training causes an increase in $\mathrm{VO}_{2 \max }$ up to approximately $25 \%$. This increase in $\mathrm{VO}_{\text {max }}$ is attributable to various factors. One is a training-induced increase in the maximal cardiac output, which is largely caused by an increase in stroke volume (2). Another adaptation of training is an increase of the oxygen extraction, resulting in an increased arterio-venous difference of oxygen content $(5,15,16,17$, $18,40)$. The increased oxygen extraction is attributed to an increased capillarization and an increase in mitochondria in the muscle (2).

\subsubsection{Iraining oi Skeietal muscie}

The number and type of muscle fibers in a specific skeletal muscle are settled it birth and hardly change during post-natal development. However, the muscle mass does increase during life. The increase in muscle mass during youth is caused by longitudinal growth and by increasing the number of myofibrils within each muscie fiber. A larger muscle diameter is associated with an increased strength (7). An increase in muscle mass, and consequently an increase in force can also be elicited by training. However, maximal force per square $\mathrm{cm}$ of muscle is hardly different between trained and sedentary individuals.

Muscle tissue is constantly renewed and remodelied. Therefore, there is a continuous degradation and rebuilding of muscle proteins. The average half-lives of rat muscle proteins vary between 12-18 days (35). In humans, estimations of protein degradation lead to an estimated half-life time of approximately 20 days (38). Sprint 


\section{Chapter 2}

training has been shown to cause a higher turnover of myosin in type II fibers; in endurance training all fiber types show this phenomenon (52). These differential adaptations are supposed to be related to selective recruitment during sprint and endurance exercise. These fiber type specific adaptive changes in muscles are also found in the metabolic machinery of muscle fiber $(4,33,34,47)$ (see below). Sprint training induces metabolic adaptations especially in type II fibers, while endurance training results in a specific metabolic adjustment in type I fibers (60). Both sprint- and endurance training result in increased amounts of mitochondrial enzymes in man as well as in horses (14, $25,42,63)$. In these animals draught training also improves the oxidative capacity and results in a lower lactate level after an identical external work load during a training period (20). The changes are most evident in the first 3-4 weeks of the training period. Training leads to an increased tissue perfusion by an increase in the number of capillaries, which reduces the diffusion distance. The increasing amount of capillaries is linearly related to increases in the $\mathrm{VO}_{2 \max }$ (see below).

\subsubsection{The effect of training on skeletal muscle energy metabolism}

One of the aims of physical training is to improve the metabolic capacity of skeletal muscles in order to enhance performance. In general, endurance training is performed to enhance aerobic ATP production in muscles used during continuous exercise, whereas sprint or high-intensity training is meant, among others, to increase the capacity and rate to produce ATP under anaerobic conditions during short term intensive exercise. Because of the complex nature of muscle energy conversion, training does most likely not affect one particular step in the various metabolic pathways. On the contrary, the whole spectrum of perfusion of the vascular bed (that means supply of nutrients and oxygen to the exercizing muscles), the amount of proteins involved in transmembrane transport, the activity of enzymes playing a key role in the metabolic pathways, mitochondrial density, the amount of carbohydrates and triacylglycerols stored in the muscle cells, and the cytoplasmic concentration of highenergy phosphates may all alter as a consequence of the training program applied. 


\subsubsection{Training and skeletal muscle perfusion}

Endurance training generally increases the degree of capillarization of skeletal muscles (51). This finding indicates that the supply of nutrients, such as glucose and fatty acids, and of molecular oxygen is improved in the endurance-trained muscle. Moreover, an increase in number of capillaries reduces the diffusion distance of the nutrients from the blood compartment to the exercising muscle cells. This might especially benefit type II fibers (51).

\subsubsection{Training and mitochondrial oxygen consumption}

A host of studies has show that mitochondrial density in muscle cells is increased as a consequence of both sprint and endurance training $(14,25,42,63)$. This effect of training has been observed in a variety of species, including man and horse: Due to the increase in number of mitochondria, the maximal oxygen consumption rate of the muscles is enhanced. Concomitantly, at comparable workloads the production of lactate is reduced in trained muscles indicating a shift from anaerobic conversion of pyruvate to lactate to aerobic degradation of pyruvate via acetyl CoA in the mitochondrial matrix. It should be realised that the degree of capillarization and the mitochondrial density are not the sole factors determining $\mathrm{VO}_{2 \max }$. Cardiac stroke volume and the oxygen carrying capacity of blood are important factors as well (2).

\subsubsection{The effect of training on muscle glucose utilisation}

Experimental studies performed during the past decade have provided conflicting findings regarding the effect of training on carbohydrate consumption in skeletal muscle. Striking species difference may exist between rat and man. In rats, daily physical training results in an increased uptake of glucose during exercise, associated with enhanced levels of GLUT4, and elevated activity of hexokinase and citrate synthase in soleus muscle (53). Other investigators (46) found in endurance-trained rats an increase of glucose uptake in both fast-twitch and slow-twitch red fibers. GLUT1 and GLUT4 content was significantly enhanced in fast-twitch red fibers. Neufer and 
Chapter 2

colleagues (44) also observed in rats a positive effect of endurance training on the expression of GLUT4 in soleus and red vastus lateralis muscle, but not in white vastus lateralis muscle. Idström and co-workers (27), however, failed to demonstrate in rats an increase of the maximal activity of glycolytic enzymes. Moreover, no differences were found in glucose transport or net glucose uptake between trained and sedentary rats.

In contrast, in man endurance training generally results in a decrease of glucose consumption of muscles during sub-maximal exercise. Both consumption of bloodborne glucose and utilisation of glucose from the endogenous glycogen store is reduced in physically trained individuals $(6,30)$, whereas $\mathrm{VO}_{2 \max }$ increased. These findings clearly indicate a carbohydrate sparing effect of endurance training. In man, the content of GLUT4 in human skeletal tissue is increased after endurance training (48). Because an enhanced glucose transporter content suggests increased glucose utilisation rather than decreased consumption of blood-borne glucose these findings may seem paradoxical at first sight. However, it cannot be excluded that the elevated content of GLUT4 in trained muscle is localised intracellularly and, hence, does not take part in glucose transport across the sarcolemma during the exercise bout. Conversely, the elevated GLUT4 content may be instrumental in replenishing the endogenous glycogen store at a greater rate in trained than in untrained muscle cells during the recovery period after the exercise bout. Moreover, enhanced utilization of fatty acids (vide infra) in endurance trained muscles might impede the intracellular utilization of glucose, providing an explanation for depressed consumption of carbohydrate in trained muscles. The mechanisms of fatty acid-mediated inhibition of muscle glucose utilization is poorly understood. Evidence of an operative glucose-fatty acid or Randle cycle in trained muscle fibers is weak (69). Further studies are required to elucidate the molecular mechanism of depressed carbohydrate consumption in skeletal muscle after endurance training. 


\subsubsection{Training and muscular fat metabolism}

As indicated above, endurance training increases the maximall oxidative capacity of the exercising muscles and causes a substantial shift from carbohydrate to fat oxidation (69). This metabolic shift in the endurance trained muscle may be caused by a variety of specific adaptations. In most studies performed in several species, endurance training increases the maximal activity of enzymes involved in fatty acid activation and B-oxidation, such as fatty acyl CoA synthetase, carnitine acyl transferase, fatty acyl CoA dehydrogenase, and 3-hydroxy- acyl CoA dehydrogenase. In the horse, Snow and Guy (54) observed a $60 \%$ increase in the activity of 3-hydroxyacyl CoA dehydrogenase in the semitendinosus muscle after endurance training. Interestingly, high intensity sprint training did not enhance the maximal activity of rat muscle 3hydroxyacyl CoA dehydrogenase (58).

Conflicting findings have been reported regarding the effect of training on fatty acid-binding protein (FABP), a cytoplasmic protein most likely involved in the transport of fatty acids from the sarcolemma to the intracellular sites. of conversion. Deggens et al. (9) reported a significant increase of the content of FABP in rat plantaris muscle, whereas Van Breda and colleagues (67) failed to observe any effect of endurance: training on the cytoplasmic FABP level in rat soleus and EDL muscle.

In addition to the maximal activity of enzymes involved in intracellular fatly acid handling, other factors may also be responsible for enhanced fat utilization in trained muscles. In this respect it is of importance to note that in trained human beings the utilization of plasma-borne fatty acids by exercising muscles is reduced rather than increased, despite a lower respiratory quotient (indicative for enhanced fat oxidation) during the exercise bout (69). This is partly due to the fact that the concentration of circulating fatty acids (complexed to albumin) is lower in trained than in untrained individuals (8). A feasible explanation for this phenomenon is that the decline in plasma fatty acid levels is caused by a decreased release of these substrates from adipose tissue due to a training-induced blunting of the plasma catecholamine 
Chapter 2

response to submaximal exercise in trained subjects. However, in spite of a decreased uptake of circulating fatty acids, the contribution of lipid oxidation to overall muscle energy conversion substantially increases in well-trained exercising individuals (39). Since most authors failed to disclose an increased utilization of circulating lipoproteintriacylglycerols in muscles subjected to endurance training (69), despite enhanced activity of lipoprotein lipase in trained muscles (31), the extra amount of fatty acids utilised in the oxidative pathway most likely originates from inter- and intramuscular fat stores. In line with this notion is the observation of Jansson and Kaijser (28), that in untrained and trained human subjects intramuscular triacylglycerol contributed by respectively $15 \%$ and $34 \%$ to total energy conversion during 60 min exercise at $60 \%$ of $\mathrm{VO}_{2 \max }$.

In addition to an enhanced capacity to oxidise endogenous fatty acids in the endurance trained skeletal muscle, resting triacylglycerol levels in skeletal muscle tissue of trained male volunteers were found to be substantially higher than in untrained persons (43). In contrast, Hurley and co-workers (26) failed to observe a significant change in the triacylglycerol content of the vastus lateralis muscle in male subjects after a strenuous training program for 12 weeks. At present, no satisfactory explanation for the deviating findings can be offered (69).

Altered concentrations of regulatory factors in the cytoplasm of trained muscle cells can also contribute to the shift from carbohydrate to fat utilization. For instance, low levels of intracellular lactate, as can be anticipated in well-trained exercising skeletal muscle fibers, might prevent the lactate-induced inhibition of carnitine acyltransferase (69). Moreover, less elevated concentrations of malonyl. CoA, a well known inhibitor of carnitine acyltransferase, in trained muscle may give rise to an enhanced flux of fatty acids through the degradative pathway (69). Finally, training might favour fatty acid oxidation at the expense of glucose utilization via the "classic" Randle cycle (69). 


\subsubsection{Training and muscle high-energy phosphates}

Although the actual turnover rate of the muscle high-energy phosphate pool is of utmost importance during heavy exercise, the absolute concentration of ATP and $\mathrm{PCr}$ most likely also play a role in cellular functioning. In man, some studies suggest that training has indeed a beneficial effect on the content of high-energy phosphates in muscle tissue. For instance, Van der Vusse and colleagues (68) found that in adult volunteers endurance training caused a slight, but significant increase in muscular $\mathrm{PCr}$ levels. In young boys, Eriksson and co-workers (11) observed a 40\% increase in muscle $\mathrm{PCr}$ content after 4 months of training. A 7-month training program caused a substantial increase in ATP concentration in human muscle (29). Others, however, failed to demonstrate a significant effect of training on muscle ATP levels (68). At present no data are available on the effect of training on high-energy phosphate stores of equine muscles.

\subsection{REFERENCES}

1. Albers B., D. Bray, J. Lewis, M. Raff, K. Roberts, J.D. Watson. Molecular biology of the cell. Garland Publishing Inc New York \& London, 1989.

2. Åstrand P.O., K. Rodahl. Textbook of Work Physiology. Physiological bases of exercise. McGraw-Hill Book. Company, New York, 1986.

3. Bergström 1.. Percutaneous needle biopsy of skeletal muscle in physiological and clinical research. Scand J Clin Invest 35: 609-616, 1975.

4. Booth F.W., D.B. Thomason. Molecular and cellular adaptation of muscle in response to exercise: perspectives of various models. Physiol Rev 71: 541-585, 1991.

5. Clausen J.P.. Effect of physical training on cardiovascular adjustments to exercise in man. Physiol Rev 57: 779-815, 1977.

6. Coggan A.R.. Plasma glucose metabolism during exercise in humans. Sports Med 11: 102 $124,1991$. 
7. Costill D.L., E.F. Coyle, W.F. Fink, G.R. Lesmes, F.A. Witzmann. Adaptations in skeletal muscle following strength training. J of Appl Physiol: Respirat Environ Exercise Physiol 46: 96-99, 1979.

8. Dalsky, G., W. Martin, B. Hurley, D. Matthews, D. Bier, J. Hagberg and J.O. Holloszy. Oxidation of plasma FFA during endurance exercise. Med Sci Sports Exerc 16: 202, 1984.

9. Deggens, H., J.H. Veerkamp, H.T.B. Van Moerkerk, Z. Turek, L.J.C. Hoofd and R.A. Binkhorst. Metabolic capacity, fibre type area and capillarization of rat plantaris muscle. Effects of age, overload and training and relationship with fatigue resistance. Int I Biochem 25: 1141-1148, 1993.

10. Devlin, T.M.. Textbook of Biochemistry. Wiley-Liss, New York, 1992.

11. Eriksson B., P. Gollnick, B. Saltin. Muscle metabolism and enzyme activities after training in boys 11-13 years old. Acta Physiol Scand 87: 485-497, 1973.

12. Essen B.. Glycogen depletion of different fibre types in human skeletal muscle during intermittent and continuous exercise. Acta Physiol Scand 103: 446-455, 1978.

13. Essen-Gustavsson B., A. Lindholm, D. McMiken, S.G.B. Persson, J. Thornton. Skeletal muscle characteristics of young standardbreds in relation to growth an early training. In: Snow D.H., S.G.B. Persson, R.J. Rose (eds): Equine Exercise Physiology, Burlington Press, Cambridge, 1983, pp. 200-210.

14. Essen-Gustavsson B., D. McMiken, K. Karlstrom, A. Lindholm, S.G.B. Persson, J. Thornton. Muscular adaptation of horses during intensive training and detraining. Eq Vet J 21: 27-33, 1989.

15. Evans D.L., R.J. Rose. Maximum oxygen uptake in racehorses: changes with training and prediction from submaximal cardiorespiratory measurements. In: Gillespie I.R., N.E. Robinson (eds): Equine Exercise Physiology 2 Edition, Edwards Brothers, Ann Arbor, USA, 1987, pp. 52-67.

16. Evans D.L., R.J. Rose. Cardiovascular and respiratory responses in thoroughbred horses during treadmill exercise. J Exper Biol 134: 397-408, 1988.

17. Evans D.L., R.J. Rose. Determination and repeatability of maximum oxygen uptake and other cardiorespiratory measurements in the exercising horse. Eq Vet J 20: 94-98, 1988.

18. Evans D.L., R.J. Rose. Cardiovascular and respiratory reponses to submaximal exercise training in the thoroughbred horse. Pflügers Arch 411: 316-321, 1988. 
19. Gottlieb M.. Muscle glycogen depletion patterns during draught work in Standardbred horses. Eq Vet J 21: 110-115, 1989.

20. Gottlieb M., B. Essen-Gustavsson, A. Lindholm, S.G.B. Persson. Effects of a Draft-Loaded Interval-Training Program on Skeletal Muscle in the Horse. I Appl Physiol 67: 570-577, 1989.

21. Guy P.S., D.H. Snow. The effect of training and detraining on muscle composition in the horse. J Physiol 269: 33-51, 1977.

22. Guyton A.C. Contraction of skeletal muscle. In: Textbook of Medical Physiology, Eighth Edition, W.B. Saunders, Philadelphia, 1991, pp. 67-79.

23. Hochachka P.W., M.S.C. Bianconcini, W.S. Parkhouse, G.P. Dobson. On the Role of Actomyosin ATPases in Regulation of ATP Turnover Rates During Intense Exercise. Proc National Ac Sci USA 88: 5764-5768, 1991.

24. Hodgson D.R., R.J. Rose, J.R. Allen, J. Dimauro. Glycogen depletion patterns in horses performing maximal exercise. Res Vet Sci 36: 169-173, 1984.

25. Hodgson D.R., R.J. Rose, J. Dimauro, J.R. Allen. Effects of training on muscle composition in horses. Am J Vet Res 47: 12-15, 1986.

26. Hurley, B.F., P.M. Nemeth, W.H. Martin III, J.M. Hagberg, G.P. Dalsky and J.O. Holloszy. Muscle triglyceride utilization during exercise: effect of training. I Appl Physiol 60: 562-567, 1986.

27. Idstrom I.P, A. Elander, B. Soussi, T. Schersten, A.C. Bylund-Fellenius. Influence of endurance training on glucose transport and uptake in rat skeletal muscle. Am I Physiol 251: H903-H907, 1986.

28. Jansson, E., L. Kaijiser. Substrate utilization and enzymes in skeletal muscle of extremely endurance-trained men. J A.ppl Physiol 62: 992-1005, 1987.

29. Karlsson J., L.O. Nordesjo. Muscle lactate, ATP, and CP levels during exercise after physical training in man. J Appl Physiol 33: 199-203, 1972.

30. Karlsson J., L.O. Nordesjo, B. Saltin. Muscle glycogen utilization during exercise after physical training. Acta Physiol Scand 90: 210-217, 1974. 
31. Kiens, B., H. Lithell, K.J. Mikines, E.A. Richter. Effects of insulin and exercise on muscle lipoprotein lipase activity in man and its relation to insulin action / Clin Invest 84: 1124$1129,1989$.

32. Komi, P.V., I. Karlsson. Skeletal muscle fiber types, enzyme activities and physical performance in young males and females. Acta. Physiol Scand 103: 210-218, 1978.

33. Lemon P.W.R.. Does Exercise Alter Dietary Protein Requirements. Adv Nutr Top Sport 32: 15-37, 1991.

34. Lemon P.W.R.. Effect of exercise on protein requirements. J Sports Sci 9: 53-70, 1991.

35. Lewis S.E.M., F.J. Kelly, D. F. Goldspink. Pre- and post-natal growth and protein turnover in smooth muscle heart and slow- and fast-twitch skeletal muscles of the rat. Biochem J 217: $517-526,1984$.

36. Lindholm A., H. Bjerneld, B. Saltin. Glycogen depletion pattern in muscle fibre of trotting horses. Acta Physiol Scand 90: 475-484, 1974.

37. Lindholm A., K. Piehl. Fibre composition, enzyme activity and concentrations of metabolites and electrolytes in muscles of standardbred horses. Acta Vet Scand 15: 287-309, 1974.

38. Lundholm K., S. Edstrom, L. Ekman, I. Karlberg, P. Walker, T. Schersten. Protein degradation in human skeletal muscle tissue: the effect of insulin, leucine, amino acids and ions. Clin Sci 60: 319-326, 1981.

39. Martin, W.H., G.P. Dalsky, B.F. Hurley, D.E. Matthews, D.M. Bier, I.M. Hagberg, M.A. Rogers, D.S. King, J.O. Holloszy. Effect of endurance training on plasma free fatty acid turnover and oxidation during exercise. Am J Physiol 265: E708-E714, 1993.

40. McAllister R.M., R.L. Terjung. Training-Induced Muscle Adaptations Increased Performance and Oxygen Consumption. J Appl Physiol 70: 1569-1574, 1991.

41. McArdle W.D., F.I. Katch, V.L. Katch. Training for anaerobic and aerobic power. In: Exercise Physiology. Energy, nutrition and human performance, Lea \& Febiger, Philadelphia, 1986, pp. 347-370.

42. Milne D.W., A.A. Gabel, W.W. Muir, R.T. Skarda. Effect of training on heart rate, cardiac output, and lactic acid in standardbred horses using a standardized exercise test. I Eq Med Surg 1: 131-135, 1977.

43. Morgan, T.E., F.A. Short, L.A. Cobb. Effect of long-term exercise on skeletal muscle lipid composition. Am | Physiol 216: 82-86, 1969. 
44. Neufer P.D., M.H. Shinebarger, G.L. Dohm. Effect of training and detraining on skeletal muscle glucose transporter (GLUT4) content in rats. Can J Physiol Pharmacol 70: 12861290, 1992.

45. Nimmo M.A., D.H. Snow. Time course of ultrastructural changes in skeletal muscle after two types of exercise. J Appl Physiol 52: 910-913, 1982.

46. Ploug T., B.M. Stallknecht, O. Pedersen, B. B. Kahn, T. Ohkuwa, J. Vinten, H. Galbo. Effect of endurance training on glucose transporter expression in rat skeletal muscle. Am J Physiol 259: E778-786, 1990.

47. Poortmans J.R.. Effects of long-lasting exercise and training on protein metabolism. In: Howald H., J. Poortmans (eds): Metabolic adaptations to prolonged physical exercise, Birkhauser, Basel, 1975, pp. 212-228.

48. Richter, E.. Glucose metabolism. In: Rowell, L.B., J.T. Shepherd (eds): Handbook of Physiology, Am Physiol Soc, Oxford Press, N.Y., 1996.

49. Sahlin K., S. Broberg, J.M. Ren. Formation of inosine monophosfate (IMP) in human skeletal muscle during incremental dynamic exercise. Acta Physiol Scand 136: 193-198, 1989.

50. Sahlin K., A. Katz. Hypoxaemia increases the accumulation of inosine monophosfate (IMP) in human skeletal muscle during submaximal exercise. Acta Physiol Scand 136: 199-203, 1989.

51. Saltin, B., P.D. Gollnick. Skeletal muscle adaptibility: Significance for metabolism and performance In: L.D. Peachy (ed.): Handbook of Physiology, Section 10. Skeletal muscle. Williams and Wilkins, Baltimore, 1983, pp. 555-631.

52. Seene T., K. Alev. Effect of Muscular Activity on the Turnover Rate of Actin and Myosin Heavy and Light Chains in Different Types of Skeletal Muscle. Int I Sports Med 12: 204-207, 1991.

53. Slentz: C.A., E.A. Gulve, K.J. Rodnick, E.J. Henriksen, J.H. Youn, J.O. Holloszy. Glucose transporters and maximal transport are increased in endurance-trained rat soleus. I Appl Physiol 73: 485-492, 1992.

54. Snow, D.H., P.S. Guy. The effect of training and detraining on several enzymes in horse skeletal muscle. Arch Int Physiol Biochem 87: 87-93, 1979. 
Chapter 2

55. Snow D.H., P. Baxter, R.J. Rose. Muscle fibre composition and glycogen depletion in horses competing in an endurance ride. Vet Rec 108: 374-378, 1981.

56. Snow D.H., P.S. Guy. Fiber type and enzyme activities of the gluteus medius in different breeds of horses. In: Poortmans, I., G. Nisert (eds): Biochemistry of Exercise, University Park Press, Baltimore, 1982, pp. 275-282.

57. Snow D.H., R.C. Harris, S.P. Gash. Metabolic response of equine muscle to intermittent maximal exercise. J Appl Physiol 58: 1689-1697, 1985.

58. Staudte, H.W., G.U. Exner, D. Pette. Effects of short-term, high intensity (sprint) training on some contractile and metabolic characteristics of fast and slow muscle of the rat. Pflugers Arch 344: 159-168, 1973.

59. Stryer L.. Biochemistry. WH Freeman and Company New York, 1988.

60. Takekura H., T. Yoshioka. Different Metabolic Responses to Exercise Training Programmes in Single Rat Muscle Fibres. J Muscle Res Cell Motil 11: 105-113, 1990.

61. Talmadge R.J., J.I. Scheide, H. Silverman. Glycogen synthesis from lactate in a chronically active muscle. J Appl Physiol 66: 2231-2238, 1989.

62. Talmadge R.J., H. Silverman. Glyconeogenic and Glycogenic Enzymes in Chronically Active and Normal Skeletal Muscle. J Appl Physiol 71: 182-191, 1991.

63. Thornton 1., B. Essen-Gustavsson, A. Lindholm, D. McMiken, S.G.B. Persson. Effects of Training and Detraining on Oxygen Uptake, Cardiac Output, Blood Gas Tensions, pH and Lactate Concentrations during and after Exercise in the Horse. In: Snow D.H., S.G.B. Persson, R.J. Rose (eds): Equine Exercise Physiology, Burlington Press, Cambridge, 1983, pp. $470-486$.

64. Valberg S., B. Essen-Gustavsson, H.S. Wallberg. Oxidative capacity of skeletal muscle fibres in racehorses - histochemical versus biochemical analysis. Eq Vet J 20: 291-295, 1988.

65. Valberg S., B. Essen-Gustavsson, A. Lindholm, S.G.B. Persson. Blood chemistry and skeletal muscle metabolic responses during and after different speeds and durations of trotting. Eq Vet J 21: 91-95, 1989.

66. Van Bilsen, M. The significance of myocardial non-esterified fatty acid accumulation during ischemia and reperfusion. Thesis University of Limburg, Maastricht, The Netherlands, 1988.

67. Van Breda, E., H.A. Keizer, M.M. Vork, D.A.M. Surtel, Y.F. De Jong, G.J. Van der Vusse, I.F.C. Glatz. Modulation of fatty-acid-binding protein content of rat heart and skeletal 
muscle by endurance training and testosterone treatment. Pflugers Arch 421: 274-279, 1992.

68. Van der Vusse G.J., G.M.E. Janssen, W.A. Coumans, H. Kuipers, R.J.M.M. Does, F. Ten Hoor. Effect of training and 15-, 25- and $42 \mathrm{~km}$ contests on the skeletal muscle content of adenine an guanine nucleotides, creatine phosphate and. glycogen. Int I Sports Med 10: S146-\$152, 1989.

69. Van der Vusse, G.J., R.S. Reneman. Lipid metabolism in muscle. In: Rowell, L.B., J.T. Shepherd (eds): Handbook of Physiology, Am Physiol Soc, Oxford Press, N.Y., 1996, pp. 952-994.

70. Weber J.M., G.P. Dobson, W.S. Parkhouse, D. Wheeldon, J.C. Harman, D.H. Snow, P.W. Hochachka. Cardiac output and oxygen consumption in exercising Thoroughbred horses. Am ! Physiol 253: R890-R895, 1987. 


\section{VARIABILITY IN FIBER TYPE COMPOSITION AND}

\section{IN THE CONTENT OF PHOSPHOCREATINE, ATP,}

NAD AND GLYCOGEN IN EQUINE GLUTEUS

\section{MEDIUS MUSCLE}

This chapter will be published as:

G. Bruin, H. Kuipers, G. André, and G.J. van der Vusse

Variability in fiber type composition and in the content of phosphocreatine,

ATP, NAD and glycogen in equine gluteus medius muscle

Int J Sports Med (Accepted for publication) 
Chapter 3 


\subsection{ABSTRACT}

Interpretation of results of histochemical or biochemical analysis of muscle biopsies may be complicated by a high variability in the values of muscle tissue parameters under investigation. This variability may be caused by factors such as local differences in fiber type composition and content of chemical compounds, and variations in the analytical techniques employed. To assess the variability of fiber type composition and content of biochemical variables, such as PCr, ATP, NAD and glycogen, between different regions and the reproducibility of tissue samples obtained from the same region, biopsies were taken from the gluteus medius muscle of Standardbred trotter stallions at three different spots at a depth of either 3 or $6 \mathrm{~cm}$.

It was observed that the variation in percentage fiber types was relatively small in the deeper layers of the gluteus medius muscle. With one exception, the mean percentage of type I and type II fibers did not differ between the various locations investigated. The variation in biochemical substances was found to be the smallest in the deeper layer of the region located about 8 to $9 \mathrm{~cm}$ ventrolateral to the middle of the straight line connecting the tuber coxae and the trochanter major. In general, the mean values of PCr, ATP, NAD, and glycogen did not significantly differ between the various regions of the muscle. However, the spot with the lowest percentage of type II fibers showed also the lowest content of PCr, ATP, NAD, and glycogen. 


\subsection{INTRODUCTION}

Since the introduction of the needle biopsy technique (1), sampling of muscle biopsies is a commonly used procedure in exercise physiology. It should, however, be realised that a biopsy is a small part from a total muscle, which may hamper a correct extrapolation of findings in the biopsy sample to the muscle in toto. Normal muscle usually contains a mixture of the two main fiber types, i.e., type I and type II. The mechanical and metabolic characteristics of both fiber types are different. Type I fibers have a lower maximal contraction speed than type II fibers (12). In addition, type I fibers display energy conversion mainly in an aerobic fashion, associated with a high resistance against fatigue, whereas type II fibers have a high anaerobic capacity and a low fatigue resistance (12).

As long as the relative distribution of both fiber types is constant throughout the muscle, biopsies from different sample sites can be compared. However, it has been shown in humans as well as in animals that the distribution of both fiber types may not be homogeneous $(2,3,12)$. Since the middle gluteal muscle is intensively used during running, it is an attractive muscle to take biopsies from in order to obtain information about cellular and biochemical changes during exercise. Therefore, the biopsies should be taken from weli defined locations where variations in fiber type composition and content of biochemical substances are as small as possible.

Because analysis of biopsy samples was an important component of this series of investigations, the aim of this study was to delineate the variability in the tissue composition of fiber types ! and II as wel! as variability in the content of glycogen and high energy phosphates in serial samples taken at well defined spots and depths in the middle gluteal muscle, in order to allow for assessment of the region of choice for taking biopsies in future experiments. 


\subsection{MATERIAL AND METHODS}

\subsubsection{Horses and Muscle Sampling}

In three Standardbred. trotter stallions, ranging in age from 3 to 5 years, serial samples were taken from the middle gluteal muscle. In each horse 3 spots were identified and at each of the three spots 3 biopsies were taken from 2 different depths, i.e., at 3 and $6 \mathrm{~cm}$. Spot 1 and 2 were chosen at a horizontal straight line through the tuber coxae at 10 and $20 \mathrm{~cm}$ behind the tuber coxae, respectively. The third spot was located about $8 \mathrm{~cm}$ ventrolaterally of the other two points. (Fig. 3.1). The samples were taken with a biopsy needle (outer diameter: $7 \mathrm{~mm}$ ), according to the technique originally described by Bergström and slightly modified by others (1, 9, 14). To increase the sample size mild suction was applied according to Evans et al. (4).

\subsubsection{Analysis of Muscle Tissue}

The portion of the biopsy for biochemical analysis was rapidly frozen in liquid nitrogen. Time interval between sampling of tissue and freezing was 5 to $10 \mathrm{~s}$. The frozen tissue samples were stored at $-80^{\circ} \mathrm{C}$. Prior to analysis the samples were freeze-dried overnight at $-30^{\circ} \mathrm{C}$.

Freeze-dried tissue was weighed and divided in at least two parts. One part (weighing 10 mg dry weight) was used for the determination of adenine nucleotides and related compounds, phosphocreatine ( $\mathrm{PCr}$ ), lactate, and glucose. For this purpose the tissue samples were extracted at $-15^{\circ} \mathrm{C}$ in a mixture of perchloric acid $(3 \mathrm{M})$ and dithiothreitol (5 $\mathrm{mM}$ ). The extraction volume was adjusted to the dry weight of the tissue specimen ( $\sim 25 \mu \mathrm{l} / \mathrm{mg}$ dry tissue weight). The tissue was ground with a glass rod in the extraction fluid and subsequently rapidly frozen in liquid nitrogen and thawed to a temperature of $4^{\circ} \mathrm{C}$. The mixture was centrifuged at $4^{\circ} \mathrm{C}$ with $1200 \mathrm{~g}$ for $5 \mathrm{~min}$. An aliquot of the supernatant was removed and neutralised with $\mathrm{KHCO}_{3}$ (20). The 
Chapter 3

neutralised supernatant was stored at $-80^{\circ} \mathrm{C}$ until further analysis.

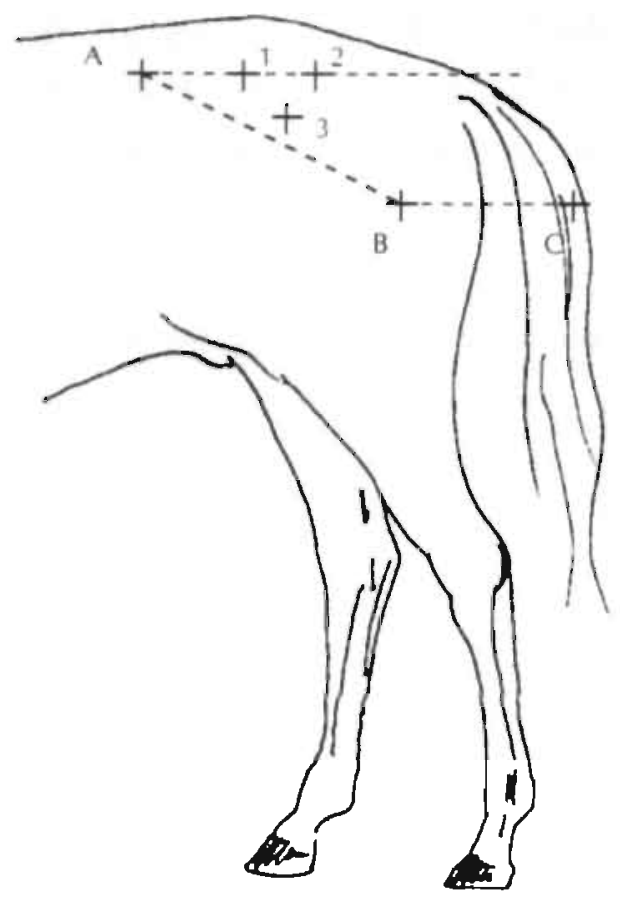

Figure 3.1 Different sample sites of the middle gluteal muscle of the horse. The meaning of the marked spots is as follows: 1. Spot 1; 2. Spot 2; 3. Spot 3; A. Tuber coxae; B. Trochanter major: C. Tuber ischiadicum

The second part of the freeze-dried tissue ( $\sim 5 \mathrm{mg}$ dry weight) was used for glycogen determination. The tissue specimens were kept at $100^{\circ} \mathrm{C}$ for $3 \mathrm{~h}$ after addition of $1.0 \mathrm{ml}$ of $1 \mathrm{M} \mathrm{HCl}$ to hydrolyse glycogen. Thereafter, the samples were neutralised with tris(hydroxymethyl)aminomethane $(0.12 \mathrm{M})-\mathrm{KOH}(2.1 \mathrm{M})$, saturated with $\mathrm{KCl}$. The glucose residues were measured fluorometrically as described elsewhere (19). The values obtained were corrected for the amount of free glucose already 
present in the tissue at the time of sampling and expressed as micromoles of glycosyl units per gram dry weight.

The tissue content of adenine nucleotides and related compounds was determined by high performance liquid chromatography with the use of a modified method after Wijnants and Van Belle (21) and described by Van der Vusse et al. (20). Briefly, a small sample of the neutralised extract $(10 \mu \mathrm{l})$ was applied to a reverse phase column (Lichrosorb RP-18, Merck). Stepwise gradient elution, using two solvents, was applied to separate the compounds of interest. Flow rate amounted to $0.8 \mathrm{ml} / \mathrm{min}$. Solvent A was an aqueous buffer of $\mathrm{NH}_{4}\left(\mathrm{H}_{2} \mathrm{PO}_{4}\right)(150 \mathrm{mM}, \mathrm{pH}=6.0)$, solvent $\mathrm{B}$ consisted of a 1:1 (vol/vol) mixture of acetonitrile and methanol. Peaks were detected at $254 \mathrm{~nm}$ and identified by comparing retention times with known standards. An example of a standard HPLC chromatogram is given in Fig. 3.2. In case of IMP, the presence of this substance in the extraction fluid was positively identified by comparing the extinction of the peak at two different wave lengths (i.e., 254 and $293 \mathrm{~nm}$ ). LiChroCart 4-4 (Merck) was used as guard column.

PCr was measured fluorometrically as described earlier (19). Lactate was assayed according to Passoneau (11). Free glucose was assayed as described before (19).

The portion of the biopsy for histochemistry was mounted on cork and frozen in isopentane, cooled to melting temperature in liquid nitrogen. The samples were stored at $-80^{\circ} \mathrm{C}$ until further processing. For histochemical staining $8 \mu \mathrm{m}$ thick serial cross sections were cut in a cryostat at $-20^{\circ} \mathrm{C}$ and mounted on cover glasses. Serial sections were stained for ATPase, after preincubation at $\mathrm{pH} 4.2$ and $\mathrm{pH} 4.6$ (12), and classified into type I, IIa, and Ilb. For identification and counting of muscle fibers pictures were made with the use of A Zeiss photo microscope. 
Chapter 3

Fig. 3.2 (For legend see next page)

Fig. 3.2a

Fig. $3.2 b$

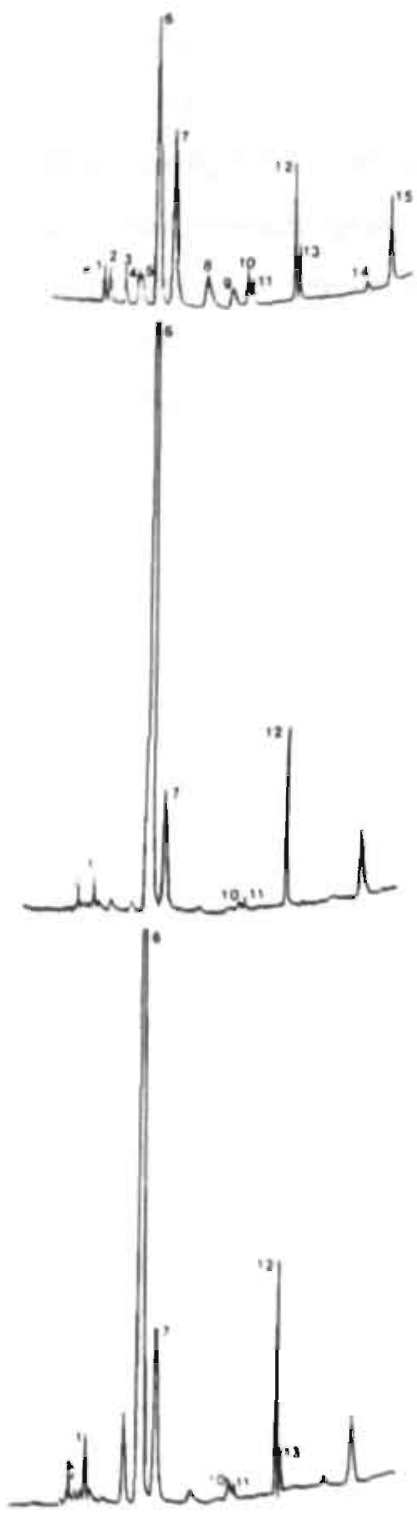

Fig. 3.2C 
Figure 3.2 Typical HPLC chromatogram of a standard solution (fig. 3.2a), extract of tissue before exercise (fig. 3.2b) and of tissue sampled immediately after an exencise bout (fig. 3.2c). The standard solution contained (in $\mu \mathrm{M}$ ):

1: GIP (7.7); 2: GDP (7.9); 3. GMP (9.0); 4. Uric acid (28.0); 5. IMP (12.9); 6. ATP (90.2); 7. ADP (72.1); 8. Hypoxanthine (31.5); 9. Xanthine (18.5); 10. AMP (9.5); 11. NADP (3.7); 12: NAD (19.6); 13. Inosine (10.7); 14. COA (9.6); 15. Adenosine (20.8). Numbers refer to numbers in chromatogram. $10 \mu$ of the standard solution was injected on column. The dry weight of tissue samples analysed in fig. $3.2 \mathrm{~b}$ and $3.2 \mathrm{c}$ was 4.3 and $4.8 \mathrm{mg}$ respectively. The gradient of the elution fluids is described in Van der Vusse et al. (19). Total run time was 28 minutes. Sensitivity of the detector was set at $0.005 \mathrm{AU} / \mathrm{mV}$.

\subsubsection{Statistical Analysis}

For comparison of various spots and depths, analysis of variance of the Genstat statistical package was used (5). Data were analysed according to the following model:

$$
y_{i j k l}=\mu+B_{i}+p_{j}+d_{k}+h_{\|}+(p d)_{j k}+(p h)_{j !}+(d h)_{k j}+(p d h)_{j k l}+\varepsilon_{i j k d}
$$

with: $\underline{y}_{i j k} \quad$ response horse $i(i=1 \ldots 3)$ at spot $j(j=1 \ldots 3)$ at depth $k(k=1,2)$ in repetition $i(i=1 . .3)$

if level

B. random horse effect, $\boldsymbol{B}-\mathrm{N}\left(0, \sigma_{p}{ }^{2}\right)$

pi spot effect

$\mathrm{d}_{\mathrm{k}} \quad$ depthi effect

$h_{1} \quad$ repetition effect

$(\mathrm{pd})_{j \mathrm{k},}(\mathrm{ph})_{j,}(\mathrm{dh})_{\mathrm{kj},}(\mathrm{pdh})_{j \mathrm{kl}}$ are 2- and 3-way interactions

$\underline{\varepsilon}_{i j|k|} \quad$ rest, $\underline{\varepsilon} \sim \mathrm{N}\left(0, \sigma_{e}{ }^{2}\right)$

The standard deviation was used as a measure of reproducibility. Differences between, spots and depths were judged by the F-test. Differences were regarded as statistically significant at the level of $\mathrm{P}<0.05$ (12). Effects of repetition were regarded as 
Chapter 3

fixed effects because influence of foregoing biopsies could not be excluded.

\subsection{RESULTS}

\subsubsection{Fiber types}

Fiber type composition at the different spots and depths are presented in the Tables 3.1 and 3.2 .

Table 3.1 Percentage of type "I fibers at different spots and depths (mean and sd).

\begin{tabular}{c|ccc|ccc}
\hline \multirow{2}{*}{ spot } & \multicolumn{3}{|c|}{ depth 1 } & \multicolumn{3}{c}{ depth 2 } \\
\cline { 2 - 7 } & \% type Ii & sd & C.V. & \% type II & sd & C.V. \\
\hline 1 & $82^{\mathrm{b}}$ & 4.3 & 5.2 & $77^{\mathrm{b}, \mathrm{c}}$ & 2.8 & 3.6 \\
2 & $63^{\mathrm{a}}$ & 12.4 & 19.6 & $81^{\mathrm{b}, \mathrm{c}}$ & 5.2 & 6.6 \\
3 & $80^{\mathrm{b}, \mathrm{c}}$ & 11.4 & 14.2 & $73^{\mathrm{c}}$ & 4.6 & 6.3 \\
\hline
\end{tabular}

Significant differences $(P<0.05)$ are indicated by the superscripts, meaning no difference in case of an identical superscript letter. Differences are indicated for spot and depth with $a, b$, and $c$.

Table 3.2 Distribution of fiber types at the different spots and depths (type lla as percentage of the total number type II fibers) (mean and sd).

\begin{tabular}{c|ccc|ccc}
\hline \multirow{2}{*}{ spot } & \multicolumn{3}{|c|}{ depth 1 } & \multicolumn{3}{c}{ depth 2 } \\
\cline { 2 - 7 } & \% type Ila & sd & C.V. & \% type Ila & sd & C.V. \\
\hline 1 & $78^{\mathrm{a}}$ & 4.9 & 6.2 & $74^{\text {a. }}$ & 4.1 & 5.5 \\
2 & $49^{\mathrm{b}}$ & 5.5 & 11.2 & $80^{\mathrm{a}}$ & 9.4 & 11.7 \\
3 & $77^{\mathrm{a}}$ & 11.2 & 14.5 & $73^{\mathrm{a}}$ & 7.4 & 10.1 \\
\hline
\end{tabular}

Significant differences $(P<0.05)$ are indicated by the superscripts, meaning no difference in case of an identical superscript letter: 
It was observed that in the middle gluteal muscle type IIA predominates, and that type IIB and type I are less prevalent. The fiber type composition was not statistically different at the different spots and depths, except for spot 2 at a depth of $3 \mathrm{~cm}$, where a lower percentage of type II and, among type II fibers, a lower percentage of type Ila fibers were found. Mean fiber type composition in biopsies from the deeper region showed the lowest standard deviation. Because of the lowest variability in fiber type composition spot 1, depth 2 appeared to be the region of choice for tissue sampling to analyse fiber composition.

\subsubsection{Tissue content of PCr, ATP, NAD and glycogen}

The content of PCr in gluteus medius muscle was in the order of 60 to 90 $\mu \mathrm{mol} / \mathrm{g}$ dry weight (Table 3.3). This finding indicates that the biopsies were adequately taken and that the time interval between removing the piece of tissue from the muscle and freezing in liquid nitrogen was short enough to prevent depletion of this relatively instable high-energy phosphate. The lowest tissue levels of $\mathrm{PCr}$ were consequently found in biopsies from spot 2, depth 1. The variation in the tissue level of $\mathrm{PCr}$ was. smallest in biopsies from spot 3, depth 2. Comparable results were obtained with ATP (Table 3.4). Only in biopsies from spot 2, depth 1 ATP levels were significantly lower than in biopsies obtained from other sites in the muscle. The same holds for NAD (Table 3.5). It has been suggested that the content of NAD in muscle cells does not vary to a great extent. When this is also true for the equine gluteus medius muscle, the conclusion has to be drawn that in tissue specimen from spot 2, depth 1 extracellular matrix components or other cells than myocytes are present to a greater extent than in biopsies obtained from other sites in the muscle. This notion is supported by the finding that also the content of glycogen, which ranges from 500 to $670 \mu$ mol glucose residues/g dry weight of tissue, is lowest in biopsies taken at spot 2, depth 1 (Table 3.6). As was the case with PCr, the variation in the content of ATP, NAD and glycogen was smallest in biopsies obtained at spot 3, depth 2 . 
Table 3.3 Content of $\mathrm{PCr}$ in equine gluteus medius muscle

\begin{tabular}{c|ccc|crr}
\hline \multirow{2}{*}{ spot } & \multicolumn{3}{|c|}{ depth 1 } & \multicolumn{3}{c}{ depth 2 } \\
\cline { 2 - 7 } & $\mathrm{PCr}$ & sd & C.V. & PCr & sd & C.V. \\
\hline 1 & $82.9^{\mathrm{a}}$ & 25.5 & 30.7 & $78.6^{\mathrm{a}, \mathrm{b}}$ & 12.0 & 15.3 \\
2 & $64.3^{\mathrm{b}}$ & 16.7 & 25.9 & $87.5^{\mathrm{a}}$ & 10.5 & 12.0 \\
3 & $82.1^{\mathrm{a}}$ & 14.9 & 18.1 & $73.1^{\mathrm{a}, \mathrm{b}}$ & 0.6 & 0.8 \\
\hline
\end{tabular}

Significant differences $(P<0.05)$ are indicated by the superscripts, meaning no differences in case of an identical superscript letter. Data are given as mean, standard deviation (sd) and coefficient of variation (C.V., \%) and expressed as $\mu \mathrm{mol} / \mathrm{g}$ dry weight.

Table 3.4 Content of ATP in equine gluteus medius muscle

\begin{tabular}{c|ccr|rrr}
\hline \multirow{2}{*}{ spot } & \multicolumn{3}{|c|}{ depth 1} & \multicolumn{3}{c}{ depth 2} \\
\cline { 2 - 7 } & ATP & sd & C.V. & ATP & sd & C.V. \\
\hline 1 & $24.0^{\mathrm{a}}$ & 4.0 & 16.6 & $23.3^{\mathrm{a}}$ & 2.4 & 10.3 \\
2 & $18.3^{\mathrm{b}}$ & 3.5 & 19.1 & $21.4^{\mathrm{a}, \mathrm{b}}$ & 2.5 & 11.6 \\
3 & $21.3^{\mathrm{a}, \mathrm{b}}$ & 3.7 & 17.3 & $21.1^{\mathrm{a}, \mathrm{b}}$ & 0.9 & 4.3 \\
\hline
\end{tabular}

Significant differences $(P<0.05)$ are indicated by the superscripts, meaning no differences in case of an identical superscript letter. Data are expressed as $\mu \mathrm{mol} / \mathrm{g} d r y$ weight. 
Table 3.5 Content of NAD in equine gluteus medius muscle

\begin{tabular}{c|ccc|ccc}
\hline \multirow{2}{*}{ spot } & \multicolumn{3}{|c|}{ depth 1 } & \multicolumn{3}{c}{ depth 2 } \\
\cline { 2 - 7 } & NAD & sd & C.V. & NAD & sd & C.V. \\
\hline 1 & $2.59^{\mathrm{a}}$ & 0.36 & 13.5 & $2.31^{\mathrm{a}, \mathrm{b}}$ & 0.23 & 10.0 \\
2 & $1.94^{\mathrm{b}}$ & 0.42 & 21.6 & $2.30^{\mathrm{a}, \mathrm{b}}$ & 0.28 & 12.2 \\
3 & $2.23^{\mathrm{a}, \mathrm{b}}$ & 0.29 & 13.0 & $2.18^{\mathrm{a}, \mathrm{b}}$ & 0.22 & 10.0 \\
\hline
\end{tabular}

Significant differences $(P<0.05)$ are indicated by the superscripts, meaning no differences in case of an identical superscript letter. Data are expressed as $\mu \mathrm{mol} / \mathrm{g} \mathrm{dry}$ weight.

Table 3.6 Glycogen content in equine gluteus medius muscle

\begin{tabular}{c|ccc|ccc}
\hline \multirow{2}{*}{ spot } & \multicolumn{3}{|c|}{ depth 1 } & \multicolumn{3}{c}{ depth 2 } \\
\cline { 2 - 7 } & Glycogen & sd & C.V. & Glycogen & sd & C.V. \\
\hline 1 & $670^{\mathrm{a}}$ & 114 & 17.0 & $672^{\mathrm{a}}$ & 68 & 10.1 \\
2 & $499^{\mathrm{b}}$ & 132 & 26.5 & $640^{\mathrm{a}}$ & 76 & 11.9 \\
3 & $592^{\mathrm{a}, \mathrm{b}}$ & 94 & 15.9 & $660^{\mathrm{a}}$ & 34 & 5.2 \\
\hline
\end{tabular}

Significant differences $(P<0.05)$ are indicated by the superscripts, meaning no differences in case of an identical superscript letter. Data are given as mean, standard deviation (sd) and coefficient of variation (C.V., \%) and expressed as $\mu \mathrm{mol} / \mathrm{g}$ dry weight.

\subsection{DISCUSSION}

To assess the effect of training, on muscular performance, the technique of taking biopsies from the muscle under investigation is nowadays a widespread procedure in exercise physiology. In order to monitor physiologically relevant changes in the skeletal muscle the site where the biopsy is sampled should be well defined and the variation 


\section{Chapter 3}

in the variables measured should be as small as possible. In the present study biopsies were taken from the gluteus medius muscle in the horse at various spots and at two different depths. The variation in fiber type composition and the variation in content of some metabolic parameters were monitored in order to assess the site of choice for taking biopsies in future experiments. In the past needle biopsies were taken from different muscles and at various sites and depths of the muscle in horses used in physiological experiments. Table 3.7 summarizes some of the studies presented in the international literature. In general, biopsies were taken from m. triceps brachii $(15,17)$, $m$. biceps femoris $(15,17), m$. longissimus dorsi $(6,17), m$. semitendinosus $(6)$ and $m$. gluteus medius $(2,6,7,9,10,14,17,18,22)$. In most studies the deeper or central parts of the muscles were used for sampling, although in general the precise site and depth where the biopsies were taken were not reported. Because in the horse the gluteus medius muscle is intensively used during running, biopsies are commonly taken from this muscle to measure changes in muscle properties as a consequence of training or strenuous exercise bouts $(6,9,16,18)$. In most studies, tissue biopsies were sampled for fiber typing. In this respect it is worth to mention that Kline and colleagues (8) and Bruce and coworkers (2) observed a higher percentage type II fibers in the superficial parts of the midcle gluteal muscle than the deeper regions. These findings could not be confirmed by the present study. On the contrary we found on one spot (spot 2, see figure 3.1) a lower percentage type II fiber in the superficial layers than in more deeper regions of the equine gluteus medius muscle. No differences were observed at other sites of the middle gluteal muscle. At present no explanation can be given for the deviant findings. In man, Saltin et al. (12) observed a tendency for a higher percentage type I fibers in the deeper part of the lateral region of the quadriceps femoris muscle. Information on the variability of the percentage of fiber types in different locations of skeletal muscle is limited. Wood and colleagues (22) reported coefficients of variation on the order of $2.7 \%$ to $5.9 \%$. Essen et al. (3) found variation coefficients ranging from $6 \%$ to $12 \%$. The present observations completely fit in those published earlier. Findings summarized in Table 3.1 and Table 3.2 indicate coefficients of variation 
ranging from $3.6 \%$ to $19.6 \%$. The lowest variation was found in biopsies taken from spot 1 at a depth of $6 \mathrm{~cm}$ (see figure 3.1 ).

Table 3.7 Choice of muscle and spot of sampling used by the different authors

\begin{tabular}{|c|c|c|}
\hline Bruce et al. (2) & m.glut.med. & over the whole muscle \\
\hline Gottlieb et al. (6) & $\begin{array}{l}\text { m.semitend. } \\
\text { m.long.dorsi } \\
\text { m.brachioceph. }\end{array}$ & $\begin{array}{l}10 \mathrm{~cm} \text { cuadodorsal to trochanter major of } \\
\text { tuber coxae at a depth of } 6 \mathrm{~cm} \\
\text { midway between tuber ischii and flexor } \\
\text { aspect of the stifle at a depth of } 3 \mathrm{~cm} \\
\text { between tuber coxae and the last rib } 5 \mathrm{~cm} \\
\text { laterally to the spinous processes of the } \\
\text { lumbar vertebrae just beneath the fascia } \\
\text { 10-13 cm cranio-dorsally to the spina } \\
\text { scapulae in the midline of the muscle }\end{array}$ \\
\hline Hodgson et al. (7) & m.glut.med. & $\begin{array}{l}15 \mathrm{~cm} \text { caudodorsal to the tuber coxae at a } \\
\text { depth of } 10 \mathrm{~cm}\end{array}$ \\
\hline Kline et al. (8) & m.glut.med. & central parts \\
\hline Lindholm et al. (9) & m.glut.med. & $\begin{array}{l}10 \mathrm{~cm} \text { caudodorsal to trochanter major of } \\
\text { tuber coxae at a depth of } 6 \mathrm{~cm}\end{array}$ \\
\hline Lopez-Rivero et al. (10) & m.glut.med. & at a depth of $5 \mathrm{~cm}$ \\
\hline Snow et al. (14) & m.glut.med. & $10 \mathrm{~cm}$ caudodorsal to tuber coxae \\
\hline Stull et al. (15). & $\begin{array}{l}\text { m.tric.brachii } \\
\text { m.biceps fem. }\end{array}$ & $\begin{array}{l}\text { central parts } \\
\text { medially in the ventral part }\end{array}$ \\
\hline Van den Hoven et al. $(17,18)$ & $\begin{array}{l}\text { m.glut.med. } \\
\text { m.long.dorsi } \\
\text { m.tric.brachii } \\
\text { m.biceps fem. }\end{array}$ & $\begin{array}{l}10 \mathrm{~cm} \text { behind tuber coxae } \\
\text { not mentioned } \\
\text { not mentioned } \\
\text { not mentioned }\end{array}$ \\
\hline Wood et al. (22) & m.glut.med. & $3-8 \mathrm{~cm}$ behind tuber coxae \\
\hline
\end{tabular}


Hence, we consider this site the region of choice in equine gluteus medius muscle to take biopsies from when the possible effect of training on changes in fiber type composition has to be studied. Data on the variation of the muscle content of relevant biochemical parameters are very scarce. The present study shows that coefficients of variation ranged from $0.8 \%$ to $30.7 \%, 4.3 \%$ to $19.1 \%, 10.0 \%$ to $21.6 \%$, and $5.2 \%$ to $26.5 \%$ for PCr, ATP, NAD, and glycogen, respectively. The smallest variation of all four variables was observed by biopsies obtained from spot 3 at depth 2 . Hence, this site is the region of choice for taking biopsies for analysis of biochemical parameters.

\subsection{REFERENCES}

1. Bergström J.. Percutaneous needle biopsy of skeletal muscle in physiological and clinical research. Scand J Clin Invest 35: 609-616, 1975.

2. Bruce V., R.J. Turek. Muscle fibre variation in the gluteus medius of the horse. Eq Vet J 17: 317-321, 1985.

3. Essen B., A. Lindholm, I. Thornton. Histochemical properties of muscle fibre types and enzyme activities in skeletal muscles of standardbred trotters of different ages. Eq Vet J 12: 175-180, 1980.

4. Evans W.M., S.K. Phinney, V.R. Young. Suction applied to a muscle biopsy maximizes sample size. Med Sci Sports Exercise 14: 101-102, 1982.

5. Genstat 5 Committee (R.W. Payne, chairman, P.W. Lane, secretary). Design and analysis of experiments. In: Genstat 5 Release 3 Reference Manual, Clarendon Press, Oxford, 1993, pp. 461-538.

6. Gottlieb M., B. Essen-Gustavsson, A. Lindholm, S.G.B. Persson. Circulatory and muscle metabolic responses to draught work compared to increased trotting velocities. Eq Vet II. 20: 430-434, 1988.

7. Hodgson D.R., R.J. Rose, J.R.Allen. Muscle glycogen depletion and repletion patterns in horses performing various distances of endurance exercise. In: Snow D.H., S.G.B. Persson, R.J. Rose (eds): Equine Exercise Physiology, Burlington Press, Cambridge, 1983, pp. $229-$ 236. 
8. Kline K.H., L.M. Lawrence, J. Novakofski, P.J. Bechtel. Changes in Muscle Fiber Type Variation within the Middle Cluteal of Young and Mature Horses as a Function of Sampling Depth. In: Gillespie J.R., N.E. Robinson (eds): Equine Exercise Physiology 2 Edition, Edwards Brothers, Ann Arbor, USA, 1987, pp. 271-277.

9. Lindholm A., K. Piehl. Fibre composition, enzyme activity and concentrations of metabolites and electrolytes in muscles of standardbred horses. Acta Vet Scand 15: 287-309, 1974.

10. Lopez-Rivero J.L., E. Aguera, M.V. Rodriguez-Barbudo, A.M. Galisteo, J.L. Morales-Lopez. Degree of Correspondence Between Contractile and Oxidative Capacities in Horse Muscle Fibres - A Histochemical Study. Histol Histopathol 5: 49-53, 1990.

11. Passoneau J.V.. Lactate. Fluorometric method. In: Bergmeyer H.U. (ed): Methods of Enzymatic Analysis, New York Academic Press, New York, 1974, pp. 1464-1472.

12. Saltin B., P.D. Gollnick. Skeletal muscle adaptibility: significance for metabolism and performance. In: L.D. Peachy (ed.): Handbook of Physiology, Section 10. Skeletal Muscle, Williams and Wilkins, Baltimore, 1983, pp. 555-631.

13. Snedecor G.W., W.G. Cochran. Statistical Methods. The lowa State University Press, Ames, lowa, USA, 1989.

14. Snow D.H., P.S. Guy. Percutaneous needle muscle biopsy in the horse. Eq Vet J 8: 150$155,1976$.

15. Stull C.L., W.W. Albert. Comparison of muscle fiber types from 2-year-old fillies of the Belgian Standardbred, thoroughbred, Quarter horse and Welsh breeds. I Anim Sci 51: 340$343,1981$.

16. Valberg S., B. Essen-Gustavsson. Metabolic Response to Racing Determined in Pools of Type I, IIA and IIB Fibers. In: Gillespie J.R., N.E. Robinson (eds): Equine Exercise Physiology 2 Edition, Edwards Brothers, Ann Arbor, USA, 1987, pp. 290-301.

17. Van den Hoven R., Th. Wensing, H.J. Breukink, A.E.F.H. Meijer, T.A.M. Kruip. Variation of fiber types in triceps brachii, longissimus dorsi, gluteus medius, and biceps femoris of horses. Am J Vet Res. 46: 939-941, 1985.

18. Van den Hoven R., A.E.F.H. Meijer, Th. Wensing, H.J. Breukink. Enzyme histochemical features of equine gluteus muscle fibers. Am J Vet Res 46: 1755-1761, 1985. 


\section{Chapter 3}

19. Van der Vusse G.J., W.A. Coumans, F.H. Van der Veen, A. Drake. ATP, creatine phosphate and glycogen content in human myocardial biopsies: markers for the efficacy of cardioprotection during aorta coronary bypass surgery. Vasc Surg 18: 127-134, 1984.

20. Van der Vusse G.J., G.M.E. Janssen, W.A. Coumans, H. Kuipers, R.J.M.M. Does, F. Ten Hoor. Effect of training and 15-, 25- and $42 \mathrm{~km}$ contests on the skeletal muscle content of adenine an guanine nucleotides, creatine phosphate and glycogen. Int J Sports Med 10: S146-\$152, 1989.

21. Wijnants J., H. Van Belle. Single-run high performance liquid chromatography of nucleotides, nucleosides and major purine bases and its application to different tissue extracts. Anal Biochem 144: 258-266, 1985.

22. Wood C.H., T.T. Ross, J.B. Armstrong, D.C. Hall. Homogeneity of muscle fiber composition in the M gluteus medius of the horse. I Eq Vet Sci 8: 294-296, 1988. 


\section{DIRECT MEASUREMENTS AND INDIRECT ESTIMATION OF MAXIMAL HEART RATE IN EXERCISING HORSES}

This chapter will be published as:

G. Bruin, H. Kuipers, G. André, and G.J. van der Vusse

Direct measurements and indirect estimation of maximal heart rate in exercising horses.

Int J Sports Med (Accepted for publication) 


\subsection{ABSTRACT}

In Standard bred trotters heart rates measured with a digital equipment (PE3000, Polar Electro) were compared with hearts rates obtained from simultaneous electrocardiogram (ecg) recordings, which were considered as "the golden standard". In addition, the repeatability of estimated maximal heart rates obtained with two different mathematical models, using heart rates and plasma lactate concentrations during submaximal exercise as input parameters, was assessed.

Forty-five Standard bred trotters performed 191 incremental exercise tests. The relationship between PE3000- and ecg-recordings ( $H_{P(3000}$ and $H_{\text {eqg }}$, respectively) was described by the regression equation $H_{\text {Pt3000 }}=0.984 . H_{\text {eqg }}\left(R^{2}=0.99\right.$, se=0.0005). The results showed that the upper measuring limit of the PE3000-recordings was 230 beats/min, while peak heart rates measured with the ecg varied from 212 to 266 beats/min.

Maximal heart rate was estimated with the use of mathematical models with heart rate and plasma lactate concentrations as input parameters measured in 251 incremental exercise tests performed with 47 Standard bred trotters. It was found that for accurate estimates of maximal heart rate at least 6 different data points are required. Each set of data points is recorded at different levels of submaximal work loads. 


\subsection{INTRODUCTION}

Enhancement of metabolic capacity, measured as the maximal oxygen uptake $\left(\mathrm{VO}_{2 \max }\right)$, is an important goal in improving the physical performance capacity of the horse (16). For increasing the aerobic capacity or $\mathrm{VO}_{2 \max }$, the exercise intensity has been shown to be the most important factor (2). However, in training often exercise intensities are used that are lower than the intensity at which $\mathrm{VO}_{2 \max }$ is reached. Therefore the exercise intensity during training at levels below $\mathrm{VO}_{2 \max }$ is often expressed as a percentage of $\mathrm{VO}_{2 \max }$ or as a percentage of power output $(2,12,17)$. When expressing the intensity as percentage of $\mathrm{VO}_{2 \max }$, maximal oxygen uptake has to be assessed. However, measurements of $\mathrm{VO}_{2 \max }$ or power output require expensive laboratory facilities and skilled personnel. Therefore $\mathrm{VO}_{2 \max }$ measurements are not generally employed by horse trainers.

In horses as well as in humans a linear relationship has been observed between heart rate and exercise intensity $(3,11,14,20,21)$, as well as between heart rate, running speed and oxygen uptake. Therefore, the heart rate and/or the running speed are often used as parameters for the exercise intensity $(2,12)$. Under standardised conditions this relationship is highly reproducible (3). When maximal exercise is used, pelatively large inter-individual differences in the highest measured maximal heart rate or peak heart rate, ranging from 225-250 beats/min are observed (1, 6, 15, 16, 19). Training does not seem to affect maximal heart rate $(6,7,13,18)$. Because of the linear relationship between heart rate and oxygen consumption also a linear relationship exists between heart rate expressed as a percentage of maximal heart rate and relative exercise intensity expressed as a percentage of maximal oxygen uptake (5).

Since heart rate monitors are commercially available heart rate is frequently used for training purposes. Some studies have been performed to compare digitally recorded heart rates with heart rates obtained with ecg measurements. Dependent on the type of equipment, the relationship between digitally recorded heart rates and 
heart rates calculated by means of a simultaneously recorded ecg was reported to be satisfactory $(4,8,19)$. However, not all studies involved maximal heart rates. In testing heart rate monitors, Foreman et al. (8) measured a highest heart rate of 200 beats/min, Sloet et al. (19) of 225 beats/min and Evans et al. (4) measured a highest heart rate of 250 beats/min. Our own observations suggest that with the PE-3000 heart rate monitor it is not possible to measure heart rates above 230 beats/min. Consequently, when the maximal heart rate exceeds the upper limit of the measuring equipment, the use of a certain percentage of the maximal heart rate, in order to control the exercise intensity, is less accurate.

Because of the linear relationship between heart rate and running speed it is theoretically possible to estimate the maximal heart rate by extrapolation of the heart rate, measured during submaximal exercise, to maximal exercise intensity. However, the relationship between heart rate and speed as well as the maximal speed depends on track conditions and can hardly be standardised. An alternative way could be to use the relationship between other physiological variables, such as plasma lactate levels, and exercise intensity. It has been shown that a non-linear relationship exists between power-output and plasma lactate concentration (12). Assuming that there is a reproducible relationship between heart rate and plasma lactate up to maximal intensity, the maximal heart rate may be estimated using a mathematical model based on the non-linear relationship between heart rate and plasma lactate.

In view of the earlier mentioned difficulties in measuring maximal heart rate under field conditions, the first purpose of this study was to assess the accuracy of a heart rate monitor PE3000 by simultaneous comparison with the ecg-recordings. Furthermore, the applicability of two different mathematical models, based on the non-linear relationship between heart rate and plasma lactate concentration, for prediction of the maximal heart rate was evaluated. 
Chapter 4

\subsection{MATERIAL AND METHODS}

Data. were collected on 2-7 year old Standard bred trotter stallions and geldings. Before participating in the experiments all the horses were housed for more than 3 months on the experimental farm. Incremental exercise tests were carried out at the Research Station for Cattle: Sheep and Horse husbandry, Lelystad, the Netherlands. Statistical analysis were carried out using Genstat (9).

\subsubsection{Measuring heart rates}

Heart rate monitor $\left(\mathrm{H}_{\mathrm{PE} 3000}\right)$ output. was compared with the ecg-data $\left(\mathrm{H}_{\text {ecg }}\right)$ (the golden standard). Data from 191 incremental exercise tests, performed with 45 Standard bred trotters, which consisted of in total 724 recordings were evaluated. Evaluation was performed using linear regression.

$$
\underline{H}_{\mathrm{Pt}, \mathrm{i}}=\beta_{1}+\beta_{2} \cdot H_{\mathrm{ecg}, 1}+\underline{\varepsilon}_{1}
$$

with:

$\mathrm{H}_{\mathrm{DE}, \mathrm{i}} \quad$ heart rate measured by means of PE3000, measurement $\mathrm{i}(\mathrm{i}=1 \ldots .724)$

$\beta_{1}, \beta_{2} \quad$ regression parameters for intercept and slope

$\mathrm{H}_{\mathrm{ecg}}, \mathrm{l}$ heart rate measured by means of an ecg

$\varepsilon_{i} \quad$ residual, $\underline{\varepsilon} \sim N\left(0, \sigma^{2}\right)$

Significant differences from 0 for the intercept and from 1 for the slope were determined with Students' t-test. $H_{\text {ecg }}$-records with more than 230 beats/min were excluded from the analysis due to the fact that the 230 is the upper measurement limit for the PE3000. Including these records would result in biased regression-coefficients. 


\subsubsection{Estimating the relationship between plasma lactate and heart rate}

The relationship between plasma lactate and heart rate was estimated using data collected in 251 incremental exercise tests, performed with 47 Standard bred trotters. During the test the treadmill had an incline of $6.25 \%$. The exercise started with $6 \mathrm{~min}$ at a speed of $4.5 \mathrm{~m} / \mathrm{s}$ and $2 \mathrm{~min}$ at $2 \mathrm{~m} / \mathrm{s}$. Thereafter the treadmill speed was set at $5 \mathrm{~m} / \mathrm{s}$ for $2 \mathrm{~min}$, after which the speed was increased every $2 \mathrm{~min}$ by $1 \mathrm{~m} / \mathrm{s}$ until the horses were unable to maintain speed. During the last 15 seconds of each workload or stage heart rate was measured and a venous blood sample was taken via a catheter in the jugular vein.

Due to the (inherently) unequal design of the incremental exercise tests there were differences in the number of animals and tests per group. Therefore a different number of heart rate / plasma lactate combinations was available for analysis, dependent of horse and test. Statistical analyses were carried out using 41, 98 and 112 tests with 3-4, 5 and 6-7 data points from 21, 35 and 31 horses, respectively. To describe the relationship between heart rate and plasma lactate concentration different models could be used. On basis of the original relationships, with increasing plasma lactate levels an asymptotic rise of the heart rate was expected. Visual control of the available experimental data confirmed this expectation. From the practical point of view, estimating the maximal heart rate should be possible with a low number of data points. A number of empirical standard nonlinear models were tested, the general mathematical descriptions of which are listed in Genstat 5 Reference Manual (9). Only the two best fitting models with two and three unknown variables are described in the present study, respectively. Both models are linear-divided-by-linear curves, producing a rectangular hyperbola (9). Depending on the number of data points $(3-4,5$ or 6-7) classes were created, i.e., class 1 , class 2 and class 3, respectively. The first model for the relationship between heart rate $(\mathrm{H}$,_beats/min) and plasma lactate concentration $(\mathrm{L}$, 
Chapter 4

$\mathrm{mM}$ ) is given by the following equation (model 1).

$$
H=\beta_{1}+\beta_{2} * H / \underline{L}
$$

or rewritten as (for fitting purpose):

$$
\underline{L}^{-1}=\beta_{2}^{-1}\left(1-\beta_{1} \underline{H}^{-1}\right)+\underline{\varepsilon}
$$

with

$\beta_{1}, \beta_{2} \quad$ regression parameters

$\underline{\varepsilon} \quad$ error component $\left(\varepsilon \sim N\left(0, \sigma_{e}^{2}\right)\right)$

(Suffices are omitted for conveniance)

If plasma lactate concentration goes to infinity, the heart rate reaches its maximum $\left(\beta_{1}\right)$. The rate of increase of the heart rate is given by $\beta_{2}$. This equation can be fitted by simple linear regression analysis to estimate the parameters $\beta_{1}$ and $\beta_{2}$.

The second model to describe the relationship can be given as (model 2):

$$
L=\beta_{1}+\frac{\beta_{2}}{l-\beta_{3} H}+\underline{\varepsilon}
$$

with:

$\beta_{1}, \beta_{2}, \beta_{3} \quad$ regression parameters

E random error component $\left(\varepsilon \sim \mathrm{N}\left(0, \sigma_{\mathrm{e}}^{2}\right)\right)$

Model 2 has an additional value for the horizontal asymptote, the basal level of plasma lactate concentration at a low level of the heart rate $(L=\beta$, for $H=-\infty)$. In this model the maximal heart rate is also defined as the rate where the plasma lactate concentration goes to infinity. This is when $\mathrm{H}$ goes to $1 / \beta_{3}$. So, $1 / \beta_{3}$ gives an estimate for the maximal heart rate. The parameters of the equation can be estimated by nonlinear regression analysis (9). 


\subsubsection{Evaluation of consistency of maximum heart rate}

After fitting model 1 and 2 per test estimates of the maximal heart rate are obtained $\left(\beta_{1}\right.$ in model 1 and $1 / \beta_{3}$ in model 2$)$. Evaluation of the consistency of estimates of maximal heart rate within and between horses was carried out performing an analysis of the estimated maximum heart rates, using the method of restricted maximum likelihood (REML). REML provides estimates of variance components for evaluating the repeatability of estimated maximal heart rates. The model can be written as (model 3):

$$
\underline{H}_{\max u i}=\mu+\underline{\text { horse }}+\varepsilon_{i j}
$$

with:

$\underline{H}_{\max , j}$ estimated maximal heart rate for horse $i(i=1 \ldots p)$ in test $j(j=1 \ldots n)$

$\mu \quad$ overall mean

horse random effect of horse i horse $\sim N\left(0 ; \sigma^{2}\right)$ )

$\varepsilon_{i j} \quad$ random error term $\left(\varepsilon_{i j} \sim N\left(0 ; \sigma^{2}\right)\right)$

Data were grouped as before, and analysis was performed for each class separately, because less consistent estimates were expected when the number of data points available per test was low. The consistency of the estimated maximal heart rate was evaluated by the intraclass correlation coefficient (i.c.c.). The i.c.c. is calculated as $\sigma_{p}^{2} /\left(\sigma_{p}^{2}+\sigma_{e}^{2}\right)$. In this special case with no fixed effects included in the model the i.c.c. is equal to the $R^{2}$ of the model. 


\subsection{RESULTS}

\subsubsection{Measurement of heart rate}

The heart rate during the treadmill test as measured with the ecg ranged from 80 beats/min during the first exercise stage to 266 beats/min during the final stage. The highest frequency that could be recorded by the PE3000 was 230 beats $/ \mathrm{min}$. The heart rate during the period of $15 \mathrm{~s}$ is estimated as the mean of three 5 -s measurements. The standard deviation of these measurements was 0.7. The PE3000 recorded all values of 232 and higher as 232 . So, the first reliable estimate of the mean heart rate was 230 beats $/ \min \left(<\left(232-2^{*} 0.7\right)\right)$. For further analysis of the relationship between PE3000 and ecg-measurements only PE3000-recordings equal to or lower than 230 beats/min were used. The heart rate measured with the ecg and with the heart rate monitor were not significantly different and could be expressed by the regression equation :

$H_{\text {PE3000 }}=0.984 . H_{\text {ecg. }} \quad\left(R^{2}=0.99 ; s e=0.0005\right)$. In figure 4.1 the heart rate values, measured with both methods, are depicted.

\subsubsection{Estimation of the maximal heart rate}

Maximal heart rates estimated from model 1 were $\sim 7-12$ beats $/ \mathrm{min}$ underestimated compared with measured values, probably caused by the omission of the additional value for the basal level of plasma lactate concentration at low heart rate (Table 4.1). Therefore model 1 is rejected as an appropriate model for describing the relationship between the heart rate and the plasma lactate concentration.

Applying model 2 no consistent bias was found. Only class 1 and 2. showed a tendency of underestimation of the maximal heart rate due to the low number of measurements of plasma lactate and heart rate. Figure 4.2 shows an example of both models for the data of one horse at one particular day. Further analysis will only be described for model $2:\left(R^{2}>0.95\right)$. 


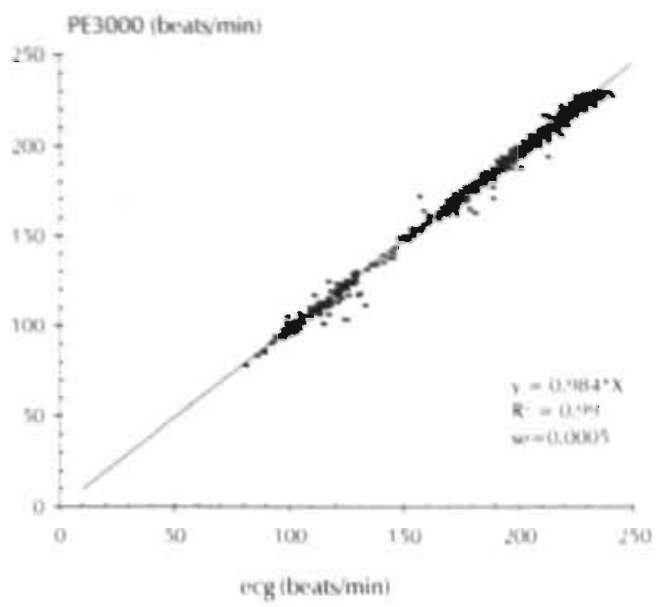

Fig. 4.1 Heart frequency registration performed simultaneously with a heart rate monitor (PE3000) and an electrocardiogram (ecg). The data are plotted as well as the regression line $y=$ $0.984 \cdot X\left(R^{2}=0.99 ;\right.$ se $\left.=0.0005\right)$.

Table 4.7 The measured peak heart rate $\left(H_{\text {measured }}\right)$ and the estimated mean maximal heart rates ( $\left.H_{\text {estimated }}\right)$ for the different classes and the number of horses for the two models used.

\begin{tabular}{|c|c|c|c|c|c|c|}
\hline Class & \multicolumn{2}{|c|}{1} & \multicolumn{2}{|c|}{2} & \multicolumn{2}{|c|}{3} \\
\hline Horses & \multicolumn{2}{|c|}{21} & \multicolumn{2}{|c|}{35} & \multicolumn{2}{|c|}{31} \\
\hline Model & 1 & 2 & 1 & 2 & 1 & 2 \\
\hline $\mathrm{H}_{\text {estimated }}$ & $239 \pm 7.6$ & $240 \pm 7.9$ & $235 \pm 7.2$ & $242 \pm 6.7$ & $233 \pm 6.1$ & $240 \pm 5.5$ \\
\hline $\mathrm{H}_{\text {measured }}$ & \multicolumn{2}{|c|}{$249 \pm 9.1$} & \multicolumn{2}{|c|}{$247 \pm 9.0$} & \multicolumn{2}{|c|}{$240 \pm 10.9$} \\
\hline
\end{tabular}




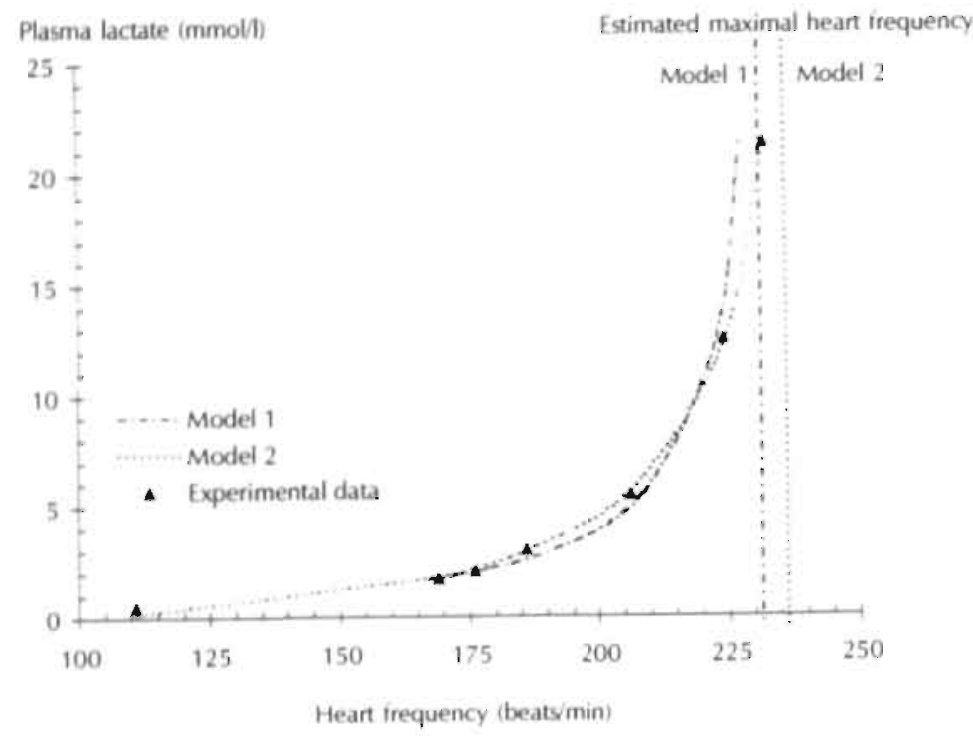

Fig. 4.2 Typical example of the estimated maximal heart rates by means of two different models (model 1 and model 2). The plotted values are the measured data of one horse at one particular test day (horse 215). The estimated maximal heart rates are presented for both models as dotted lines.

The intra class correlation varied from 0.09 for class 1 to 0.50 for class 3 (Table 4.2), meaning a higher consistency of the estimated maximal heart rate in class 3 . Therefore, using mode! 2. and having at least 6 data points available (class 3) a more accurate estimate of the maximal heart rate can be made compared to class 1 and 2 . The standard error of the estimates (= square root of the variance within horses) was 7.90 and 5.45 beats/min in class 1 and 3 respectively (Table 4.2 ). This means that the intervals $H_{\text {estimated }} \pm 16$ and $H_{\text {estimated }} \pm 11$ beats/min in class 1 and 3 (in which $H_{\text {estimated }}$ is the estimated maximal heart rate), respectively, will include the true maximal heart rate with a chance of $95 \%$. The coefficient of variation ranges from $3.30 \%$ in class 1 to $2.27 \%$ in class 3 . 
Table 4.2 Components of the REML-analysis (square root of the variance between horses $\left(s_{p}\right)$ and within horses $\left.\left(s_{p}\right)\right)$, intraclass correlation coefficients $\left(\rho_{i}\right)$, and the coefficient of variation (c.v.) for model 2.

\begin{tabular}{l|ccc}
\hline Class & 1 & 2 & 3 \\
\hline$\sigma_{p}$ & 2.51 & 4.38 & 5.47 \\
$\sigma_{e}$ & 7.90 & 6.70 & 5.45 \\
$c . v$. & 3.30 & 2.77 & 2.27 \\
$\rho_{i}$ & 0.09 & 0.30 & 0.50 \\
\hline
\end{tabular}

\subsection{DISCUSSION}

In horse training heart rate is one of the easiest measurable parameters for use in a daily routine for estimating exercise intensity. Submaximal heart rates can be measured reliably and accurately by means of the PE3000, a digital heart rate monitor, commonly used under experimental and field conditions. However, the upper limit of heart rates that could be measured with the PE3000 was 230 beats/min, while with ecg heart rates up to 266 beats/min were measured. Evans et al. (4) and Sloet et al. (19), reported similar results with a heart rate monitor of an older type than the PE3000, i.e., the Horse Tester $^{R}$. However, with newer types of heart rate monitors the accuracy at high frequencies was not tested in horses. In humans, Karvonen et al. (10) observed a higher heart rate by means of the PE3000 compared with the ecg results.

The slightly higher heart rates as measured with the PE3000 can be explained by the way of visually counting the R-peaks in the ecg during a period of 15 seconds and afterwards multiplying with 4, regardless of the heart frequency. The PE3000 


\section{Chapter 4}

calculates a running mean of the last 4 heart beats, resulting in a shorter measuring period at higher frequencies. Visual counting of R-peaks from the ecg may lead to a tendency of overestimating the heart rate by counting up to 1 extra peak, resulting in a higher heart rate. Heart rates exceeding 230 beats/min cannot be measured with the PE3000. in our experiments the range of the highest measured or peak heart rates of individual horses varied between 212 beats $/ \mathrm{min}$ and 266 beats/min with a mean of $243 \pm 11$ beats $/ \mathrm{min}$, as measured from the ecg-recordings. The mean measured peak heart rates from the animals after grouping according to the number of data points varied from 240-249 beats/min (Table 4.1). So, in most horses, the heart rates attained during maximal exercise could not be measured by using the PE3000. In humans the highest measured heart rates usually do not exceed 220 beats $/ \mathrm{min}$, a level well within the range that can be measured reliably with the PE3000. However, in horses higher frequencies can be reached, which makes measurement of maximal heart rate with this type of equipment impossible. Consequently, in practical horse training heart rate registrations for monitoring training intensity under field conditions, can be only used as long as the target heart rate does not exceed 230 beats/min.

This study shows that the individual maximal heart rate of a horse can be estimated using a mathematical model that describes the relationship between heart rate and plasma lactate (model 2, vide supra). When in this model, based on the hyperbolic relationship between plasmal lactate concentration and heart rate, the fitted curve of the plasma lactate concentration approaches infinity, the corresponding heart rate is considered as maximal heart rate. The accuracy of the predicted heart rate increased with the number of submaximal data points. The results indicate that performing an exercise test with at least 6 data points yields a reliable prediction of the maximal heart rate.

Assuming that the level of the maximal heart rate is constant, the variation in the estimated maximal heart rate is due to uncertainty in fitting the curve. One of the possible factors which may affect the accuracy of the fit, and therefore of the estimated 
maximal heart rate, is the heart rate during the first step of the exercise test. These low heart rates do not reliably reflect exercise intensity ( 2 ). Therefore, predictions of the maximal heart rate can be optimized by using heart rates obtained at a higher level of exercise intensity.

In conclusion, the present study demonstrates that the heart rate of horses can be measured accurately under field circumstances with a sport tester PE3000 up to frequencies of $230 / \mathrm{min}$. The maximal heart rate can also be estimated accurately by a mathematical model which uses heart rate and plasma lactate levels measured during at least 6 different submaximal exercise intensities, as input parameters.

\subsection{REFERENCES}

1. Asheim A., O. Knudsen, A. Lindholm, O, Rülcker, B. Saltin. Heart rates and blood lactate concentrations of standardbred horses during training and racing. I Am Vet Med Assoc 157: 304-312, 1970.

2. Åstrand P.O., K. Rodahl. Textbook of Work Physiology. Physiological bases of exercise. McGraw-Hill Book Company, New York, 1986.

3. Ehrlein H.J., W. Hörnicke, W. von Engelhardt, G. Tolkmitt. Heant frequency during standardised exercise as a measure of the working capacity of horses. Zentralbi Vet Med 20: 188-208, 1973.

4. Evans D.L., R.J. Rose. Methods of investigation of the accurancy of four digitally-displaying heart rate meters suitable for use in the exercising horse. Eq Vet J 18: 129-132, 1986.

5. Evans D.L., R.J. Rose. Maximum oxygen uptake in racehorses: changes with training and prediction from submaximal cardiorespiratory measurements. In: Gillespie J.R., N.E. Robinson (eds): Equine Exercise Physiology 2 Edition, Edwards Brothers, Ann Arbor, USA, 1987, pp. 52-67.

6. Evans D.L., R.!. Rose. Cardiovascular and respiratory reponses to submaximal exercise training in the thoroughbred horse. Pflügers Arch 411: 316-321, 1988. 
7. Evans D.L., R.J. Rose. Cardiovascular and respiratory responses in thoroughbred horses during treadmill exercise. J Exper Biol 134: 397-408, 1988.

8. Foreman J.H., D. Rabin. Determination of accuracy of a digitally displaying equine heart rate meter. I Eq Vet Sci 4: 164-163, 1984.

9. Genstat 5 Committee (R.W. Payne, chairman, P.W. Lane, secretary). Design and analysis of experiments. In: Genstat 5 Release: 3 Reference Manual, Clarendon Press, Oxford, 1993, pp. $359-583$.

10. Karvonen J., J. Chwalbinska-Moneta, S. Saynajakangas. Comparison of heart rates measured by ECG and microcomputer. Physic Sport Med 12: 65-69, 1984.

11. Lindholm A., B. Saltin. The physiological and biochemical response of standardbred horses to exercise of varying speed and duration. Acta Vet Scand 15: 310-324, 1974.

12. McArdle W.D., F.I. Katch, V.L. Katch. Training for anaerobic and aerobic power. In: Exercise Physiology. Energy, nutrition and human performance. Lea \& Febiger, Philadelphia, 1986.

13. McDougal K.G., D. Allen. Effects of intense training on maximal heart rate. Int J Sports Med 7: 177-184, 1986.

14. Persson S.G.B.. On blood volume and working capacity in horses. Acta Vet Scand Suppl 19:12-180, 1967.

15. Persson S.G.B., B. Essen, A. Lindholm. Oxygen uptake, red-cell volume, and pulse/work relationhip in different states of training in trotters. Proc Fifth Meeting Acad Soc Large Animal Vet Med, Glasgow: 34-43, 1980.

16. Persson S.G.B.. Evaluation of Exercise Tolerance and Fitness in the Performance Horse. In: Snow D.H., S.G.B. Persson, R.J. Rose (eds): Equine Exercise Physiology: 441-457, Burlington Press, Cambridge, 1983.

17. Saltin, B., P.D. Gollnick. Skeletal muscle adaptibility: Significance for metabolism and performance In: L.D. Peachy (ed.): Handbook of Physiology, Section 10. Skeletal muscle. Williams and Wilkins, Baltimore, 1983, pp. 555-631.

18. Seeherman H.I., E.A. Morris. Comparison of Yearling, 2-Year-Old and Adult Thoroughbreds Using a Standardised Exercise Test. Eq Vet J 23: 175-184, 1991.

19. Sloet-Van Oldruitenborg-Oosterbaan M.M., R. van den Hoven, H.J. Breukink. The accuracy of 3 different heart rate meters used for studies in the exercising horse. I Vet Med 
35: $665-672,1988$.

20. Thomas D.P., G.F. Fregin, N.H. Gerber, N.B. Ailes. Cardiorespiratory adjustments to tethered-swimming in the horse. Pflùgers Arch 385: 65-70, 1980.

21. Thomas D.O., G.F. Fregin. Cardiorespiratory and metabolic responses to treadmill exercise in the horse. J Appl Physiol: Resp Environ Exercise Physiol 50: 864-868, 1981. 
Chapter 4 


\section{ADAPTATION AND OVERTRAINING IN HORSES}

\section{SUBJECTED TO INCREASING TRAINING LOADS:}

IN SEARCH FOR EARLY MARKERS OF

\section{OVERTRAINING}

This chapter has been published in:

G. Bruin, H. Kuipers, H.A. Keizer, and G.J. van der Vusse

Adaptation and overtraining in horses subjected to increasing training loads.

J Appl Physiol 76: 1994, 1908-1913 


\subsection{ABSTRACT}

To evaluate markers or warning signs for overtraining syndrome, seven male race horses were subjected to 272 days of training consisting of daily exercise bouts of either endurance running (heart rate 140/min) or interval training (maximal heart rate), both increasing in duration and intensity. An incremental exercise test was held every $4 \mathrm{wk}$, and from day 187 it was held every 2 wk. Muscle glycogen, muscle lactate, energy-rich phosphates, adrenal response to adrenocorticotropic hormone, plasma and red blood cell volumes, and a number of blood chemical variables were measured. The horses showed symptoms of weight loss, irritability, and an inability to complete the training after the intensity of the endurance exercise was increased. However, test performance was not decreased. The adrenal response to adrenocorticotropic hormone was not changed when the animals showed symptoms of overtraining. The decline in muscle ATP concentration during maximal exercise was less during the period of staleness, whereas plasma volume, red blood cell volume, and blood chemical variables were unchanged. It is concluded that as long as exhaustive training is alternated by light exercise, overtraining can be avoided. Furthermore, no single parameter measured in blood could be used as warning sign to detect the onset of early overtraining. 


\subsection{INTRODUCTION}

The goal of athletic training is to increase the performance capacity by gradually increasing both intensity and volume of the training. Each exercise bout induces an acute disturbance of homeostasis in cells and organs, which is related to fatigue. In the recovery phase homeostasis has to be re-established. Regenerative processesi continue after restoration of the homeostatic situation, resulting in an overcompensation (21). Because: little is known about the optimal amount of training and the factors that influence recovery and supercompensation, training has an empirical rather than a scientific basis. It is the art of coaching to provide the optimal amount of training without exceeding an individual's exercise tolerance and recovery capacity. in all instances where recovery is incomplete and premature fatigue is encountered, one speaks of overtraining. However, overtraining is a general term and does not differentiate between the stages of overtraining. When insufficient rest is allowed for the next training or competition, recovery is incomplete and premature fatigue may occur. This condition is referred to as short-term overtraining or overreaching (9) and is quickly reversible with 1 or 2 days of little or no training. When the imbalance between training and recovery lasts for a longer period of time, a condition referred to as overtraining syndrome or staleness $(5,9)$ i may be encountered. Signs associated with staleness are increased fatigability and endocrine, metabolic, and behavioural changes $(5,9,11,13,16)$. Staleness is not easily reversible and may take several weeks to months to recover from (9).

Overtraining is found in humans, but overreaching and staleness are also frequently observed in race horses $(16,17)$. The transition from short-term overtraining into staleness develops gradually, and little is known about the underlying pathophysiological mechanism and how the transition from overreaching into staleness develops. For human and animal sport practice it is important to prevent staleness or to detect it in its earliest stage. Several measurements have been suggested and used to detect overreaching and staleness (for review see Refs. 5, 9). Blood chemical changes that indicate overreaching are increased plasma urea and creatinine levels, and increases 
in creatine kinase $(C K)(5,9)$. With staleness these changes are supposed to be supplemented with behavioural changes, decreased appetite, weight loss, decrease in aerobic power, and altered blood levels of cortisol and catecholamine (18). Barron et al. (1) presented evidence that staleness in humans is associated with a neuroendocrine disorder located at the hypothalamic level. Persson et al. (16) reported changes in the adrenal response to adrenocorticotropic hormone (ACTH) and an increased red blood cell volume in horses suffering from staleness. However, most of these blood chemical changes are unspecific, and in neither humans nor animals has a simple and unequivocal measurement been identified to detect overreaching and staleness. In addition, little information is available as to how the before mentioned parameters change during the transition from overreaching to staleness.

The aim of the present study was to investigate physical performance capacity and physiological adaptation to a training protocol consisting of exercise that gradually increased in volume and intensity and to re-evaluate potential markers or warning signs that may indicate overreaching and staleness in horses. The horse is a born athlete that shows a training response similar to humans. In addition, training on a treadmill allows the investigator to control the training while the size of the animal permits repeated sampling of blood and muscle tissue. Various measurements were done repeatedly: exercise tests, blood volume, and blood chemical variables such as urea, creatinine, CK, and the adrenal response to an $\mathrm{ACTH}$ challenge. To relate possible changes in performance and fatigability to biochemical changes in the muscles or to muscle damage, muscle biopsies were taken for determination of muscle glycogen, lactate, energy-rich phosphates, and muscle histology. 


\subsection{MATERIALS AND METHODS}

\subsubsection{Animals}

Seven 3- to 5-year-old Standardbred trotters entered the study. To avoid drop out. because of self-inflicted injuries, the animals were tested on the ability to maintain coordination at high treadmill speeds. All animals were stallions with an initial mean body weight of $416 \pm 24$ (SD) $\mathrm{kg}$. During the 3 months preceding the present study, the horses performed light aerobic exercise consisting of walking and trotting for 30-45 min/day, 5 days/wk.

The animals were individually housed in boxes, allowing social contact, and were fed at libitum with hay and straw, and with carbohydrate-rich grains, according to Dutch standards for race horses.

\subsubsection{Training}

The training period lasted 272 days, and all the training and testing was done on a treadmili (Sato). The horses exercised 7 days/wk throughout the entire training period. The training program consisted of two types of exercise, endurance running and interval running. The days of interval running were alternated with days of endurance running. To enable an exercise intensity adjusted to the individual's abilities, the running speed at which heart rates of 140,180 , and 200 beats/min were reached (v140, v180, v200) were measured during each exercise test. The training intensity was adjusted according to the most recently measured v140, v180, and v200 during the incremental exercise test. The endurance running included $20 \mathrm{~min}$ of continuous level running at v140.

Each interval training started with a 6-min warmup at v140, (mean treadmill speed $6.0 \pm 0.5 \mathrm{~m} / \mathrm{s}$ ), followed by four 3-min bouts at v200 (mean treadmill speed $9.0 \pm 0.5 \mathrm{~m} / \mathrm{s}$ ), and interspersed with 3 -min periods at v140. After 4 and 8 wk the number of 3-min bouts was increased to five and six, respectively. The total duration at 
v140 varied from $18 \mathrm{~min}$ ( 4 bouts) to $24 \mathrm{~min}$ ( 6 bouts). During each interval training the treadmill had a $6.25 \%$ incline.

At day 187 the severity of the training program was increased by increasing the total amount of work of each interval training. This was achieved as follows: training was increased to four 3-min bouts at v200 (interspersed with $3 \mathrm{~min}$ at v140), and four $60-\mathrm{s}$ all-out sprints, with $4 \mathrm{~min}$ of recovery at v140 between the sprints, were added. The incline of the treadmill was increased to $10 \%$ in order to reduce the risk of self-inflicted injuries. After 2 and 4 wk the number of 60 -s sprints was increased to five and six, respectively. The duration and intensity of the endurance running ( $20 \mathrm{~min}$ at v140) remained the same. Because the anticipated decrement in performance did not occur with this intensified interval training, at day 261 the severity of the training program was increased again by also increasing the exercise intensity of the endurance running from $20 \mathrm{~min}$ at v140 to $20 \mathrm{~min}$ at v180. Ten days after the intensity of the endurance exercise was increased, the training was discontinued because the horses showed progressive and marked irritability and increased difficulty in completing the training sessions. In addition, they did not consume the usual amount of food provided.

\subsubsection{Incremental exercise tests and procedure}

Every 4 wk and from day 187 every 2 wk the horses were subjected to an incremental exercise test on the treadmill (Sato). During the exercise test the treadmill had an incline of $6.25 \%$. The exercise test started with a warmup for $6 \mathrm{~min}$ at a treadmill speed of $4.5 \mathrm{~m} / \mathrm{s}$ and $2 \mathrm{~min}$ at $2 \mathrm{~m} / \mathrm{s}$. Thereafter the treadmill speed was set at $5 \mathrm{~m} / \mathrm{s}$ for 2 min, after which the speed was increased every $2 \mathrm{~min}$ by $1 \mathrm{~m} / \mathrm{s}$, until the animal was unable to maintain the speed at the final stage because of exhaustion. The maximal attained speed ranged from 10 to $12 \mathrm{~m} / \mathrm{s}$. Because the protocol of the incremental exercise test was identical each time, the total running time calculated from the heart rate registration was taken as measurement of performance. During the exercise tests the air temperature and relative humidity averaged $12.0 \pm 2.5^{\circ} \mathrm{C}$ and $89 \pm 9.7 \%$, respectively. 


\section{Chapter 5}

Before each test the animals were weighed and a catheter was inserted into the jugular vein for sampling of blood for the determination of plasma lactate and plasma activity of CK. A percutaneous needle biopsy was taken from the middle gluteal muscle for analysis of resting glycogen, energy-rich phosphates, and related compounds and for histological examination. The site of the biopsy was chosen midway on the imaginary line between the tuber coxae and the trochanter major and at $6 \mathrm{~cm}$ depth.

Before the exercise test was started and during the last $15 \mathrm{~s}$ of each stage, blood was drawn for assessment of plasma lactate (Lactate analyser 640 , Roche). Heart rate was recorded continuously with a heart rate monitor (PE3000).

A second muscle biopsy from the middle gluteal muscle was taken within $15 \mathrm{~s}$ after the exercise test was terminated. The sample was separated into two portions. One portion was quickly frozen in liquid nitrogen and used for determination of energy-rich phosphates and glycogen; the other portion, used for histological examination, was frozen in isopentane cooled to melting point in liquid nitrogen.

\subsubsection{Determination of plasma and red blood cell volumes}

Because the hematocrit is maximal after exertion, immediately after the exercise was stopped, plasma and red blood cell volumes were measured with the Evans blue dilution technique (15). After $15 \mathrm{~min}$ a blood sample was drawn to measure the dilution of the dye. Plasma volume (PV) was calculated according to the equation $\mathrm{PV}=\mathrm{EB} / \mathrm{C}$, in which $\mathrm{EB}$ is the amount of Evans Blue administered (in $\mathrm{mg}$ ) and $\mathrm{C}$ is the plasma concentration of the dye $(\mathrm{mg} /)$ ). Hematocrit $(\mathrm{Hct})$ was assessed in each blood sample. Red blood cell volume (RCV; $\mathrm{ml} / \mathrm{kg}$ body $\mathrm{wt})$ was calculated according to: $R C V=(P V \times$ $\mathrm{Hct}) /((100-\mathrm{Hct}) \times$ body $\mathrm{wt})$. 


\subsubsection{Histology and assay of metabolites in muscle tissue}

From the muscle sample for histology $10-\mu$ m-thick cross sections were cut in a cryostat at $-20{ }^{\circ} \mathrm{C}$ and processed for histological examination (hematoxylin-eosin) as described by Dubowitz (3).

The freeze-dried sample for biochemical analysis was weighed and divided into at least two parts. One part (weighing $\sim 10 \mathrm{mg}$ dry wt) was used for determination of adenine nucleotides and the related compounds phosphocreatine ( $\mathrm{PCr}$ ), lactate, and glucose. To this end, the tissue sample was extracted at $-15^{\circ} \mathrm{C}$ in a mixture of perchloric acid $(3 \mathrm{M})$ and dithiothreitol $(5 \mathrm{mM})$. The extraction volume was adjusted to the dry weight of the tissue specimen $(\sim 25 \mu \mathrm{l} / \mathrm{mg}$ dry tissue wt). The tissue was ground with a glass rod in the extraction fluid, rapidly frozen in liquid nitrogen, and thawed to a temperature of $4{ }^{\circ} \mathrm{C}$. The mixture was centrifuged at $4{ }^{\circ} \mathrm{C}$ at $1,200 \mathrm{~g}$ for $5 \mathrm{~min}$. An aliquot of the supernatant was removed and neutralised with $\mathrm{KHCO}_{3}$ (19). The neutralised supernatant was stored at $-80^{\circ} \mathrm{C}$ until further analysis.

The second part of the freeze-dried tissue ( $~ 5 \mathrm{mg}$ dry wt) was used for glycogen determination. The tissue specimen was kept at $100{ }^{\circ} \mathrm{C}$ for $3 \mathrm{~h}$ after addition of $1.0 \mathrm{ml}$ of $1 \mathrm{M} \mathrm{HCl}$ to hydrolyse glycogen. Thereafter, the samples were neutralised with tris(hydroxymethyl)aminomethane $(0.12 \mathrm{M})-\mathrm{KOH}(2.1 \mathrm{M})$ saturated with $\mathrm{KCl}$. The glucose residues were measured fluorometrically as described elsewhere (19). The values obtained were corrected for the amount of free glucose already present at the time of tissue sampling (see below):

The tissue contents of adenine nucleotides and related compounds were determined by high-performance liquid chromatography by use of a modified method after Wynants and Van Belle (22), and described by Van der Vusse et al. (20). Briefly, a small sample of the neutralised extract $(10 \mu \mathrm{l})$ was applied to a reversed-phase column (Lichrosorb RP-18, Merck). Stepwise gradient elution, using two solvents was applied to separate the compounds of interest. Flow rate amounted to $0.8 \mathrm{ml} / \mathrm{min}$. Solvent $A$ was an aqueous buffer of $\mathrm{NH}_{4}\left(\mathrm{H}_{2} \mathrm{PO}_{4}\right)(150 \mathrm{mM}, \mathrm{pH}=6.0)$, solvent $\mathrm{B}$ consisted of a $1: 1$ (vol/vol) 
Chapter 5

mixture of acetonitrile and methanol. Peaks were detected at $254 \mathrm{~nm}$ and were identified by comparing retention times with known standards. LiChroCART 4-4 (Merck) was used as guard column. PCr was measured fluorometrically as described earlier (19). Lactate was assayed according to Passoneau (14). Free glucose was assayed as described before (19). Glycogen content was expressed as micromoles of glycosyll units per gram dry weight.

\subsubsection{Adrenal response to $\mathrm{ACTH}$}

To study the adrenal response to ACTH, 1 day before each incremental exercise test, 25 IU of ACTH were injected intramuscularly (Synacten, equivalent to 25 IU of ACTH) between 8 and 12. a.m. Care was taken that each horse was always tested at the same time of the day. Blood was sampled for cortisol determination immediately before injection and 2 and $4 \mathrm{~h}$ later. Concentrations of cortisol were determined by radioimmunoassay with a commercial kit (D.P.C. kit, Laboratory Service). The analytic coefficients of variance were $8.2 \%$ at $120 \mu \mathrm{M}$ and $3.8 \%$ at $820 \mu \mathrm{M}$.

\subsubsection{Blood chemical variables}

To seek for parameters that may indicate overreaching or staleness, venous blood was drawn each morning from day 187 . The plasma samples were analysed for concentrations of urea and creatinine (autoanalyzer Ilc-plus, Technicon Instruments). The plasma activity of the muscle enzyme CK (E.C. 2.7.3.2) was determined with a centrifugal analyser (Cobas Bio, Hoffmann La Roche).

\section{$\underline{5.3 .8 \text { Statistical analysis }}$}

The data were analysed for differences in time with the Genstat package using repeated measures analysis of variance. Differences were located using the NewmanKeuls post hoc test. Differences were considered to be statistically significant at $\mathrm{P}<0.05$. 


\subsection{RESULTS}

\subsubsection{Training and symptoms of overtraining}

Until day 261 all the horses were able to complete the training sessions. After that day the intensity of the endurance training was increased from v140 to v180. The following two training sessions (day 261 and 263) were completed by all horses, although the animals showed difficulty in doing so. On day 267 only one animal could complete the interval training, and on day 269 all the horses failed to complete the training. In addition to the inability to complete the training, the horses developed sluggishness and increased irritability, were not able to consume the regular amount of food provided, and lost weight (Table 5.1). These symptoms were considered to reflect overtraining syndrome or staleness.

\subsubsection{Exercise test, plasma lactate, and plasma and red blood cell volumes}

The performance capacity in the incremental exercise test was expressed as total running time until exhaustion. An increase in running time was seen until 230 days (Table 5.1). When the horses showed symptoms of overtraining, no significant decline in performance on the treadrnill (day 272) was observed. The mean plasma lactate curve showed a shift to the right during the initial $8 \mathrm{wk}$ and then remained unchanged for the rest of the study (Fig. 5.1). The mean maximal levels, measured at the point of stopping the exercise, ranged from $20.3 \pm 2.4$ and $27.2 \pm 3.9 \mathrm{mM}$ in the period between day 187 and day 272 , respectively. The maximal venous hematocrit ranged from $53 \%$ to $69 \%$. with a mean of $60 \pm 1 \%$, and no relationship was observed between hematocrit and symptoms of staleness. Mean plasma volume of the animals at the start of the training period was $24.0 \pm 0.6$ litres and did not change significantly during the entire period of time. 
Chapter 5

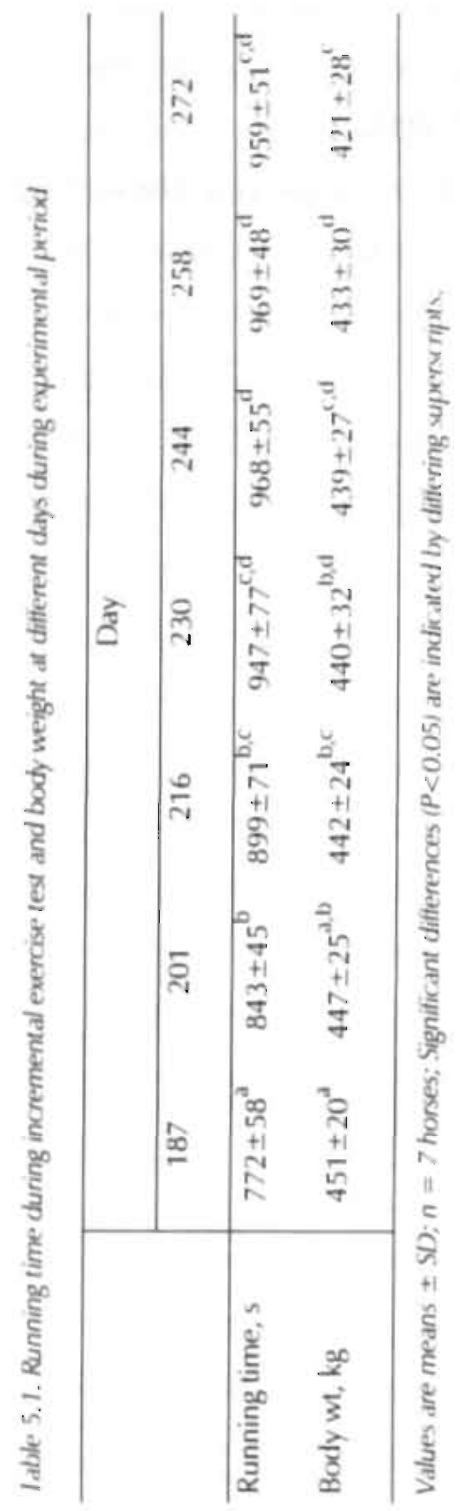


Mean red blood cell volume was $83.4 \pm 4.2 \mathrm{ml} / \mathrm{kg}$ and failed to demonstrate significant changes throughout the training period. Only on day 258 was a transient increase of plasma and red blood cell volumes found, which was not related with symptoms of overtraining.

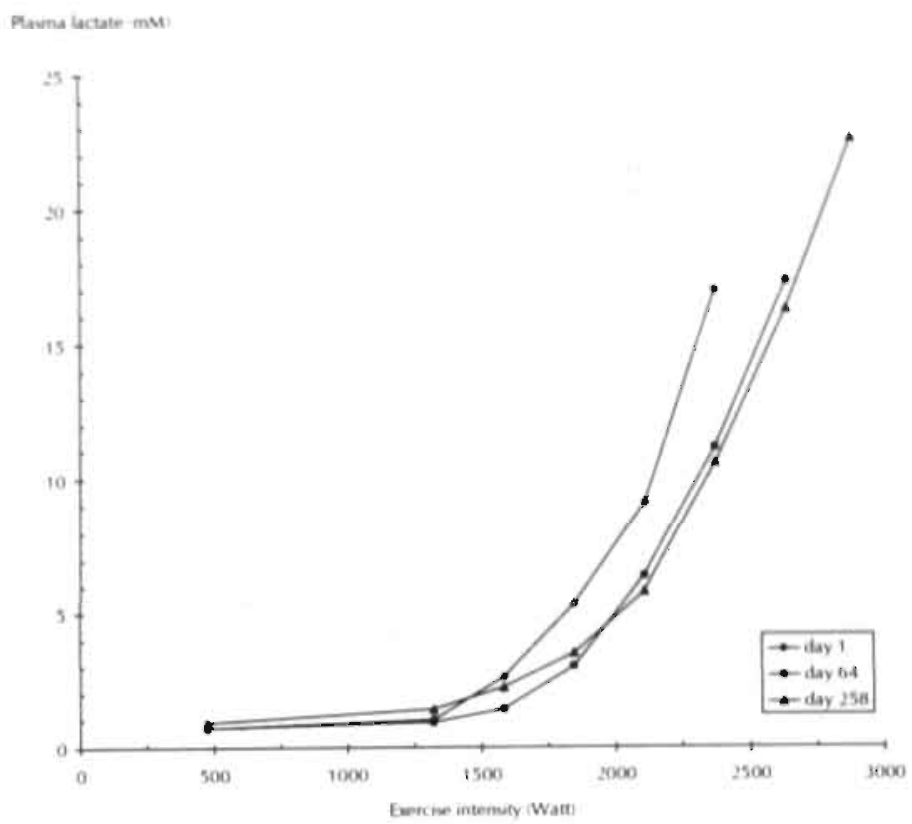

Fig. 5.1 Average plasma lactate content during the incremental exercise tests on day 1, day 64 and day 258 of the experimental period. For the sake of clarity only mean values are given $(n=7)$

5.4.3 Muscle histology, muscle glycogen, energy-rich phosphates, and muscle lactate content

Histological examination of biopsies taker, throughout the experimental period failed to revea! any degenerative or inflammatory changes that could be associated with exercise-induced muscie damage (data not shown).

Muscle glycogen levels before the start of the incremental exercise test were similar throughout the whole period (Table 5.2). Glycogen levels were decreased in muscle samples taken after the incremental exercise test (Table 5.3). 
Table 5.2. Resting muscle content of Cly, Lac, IMP, PG, AIP, ADP, and AMP before the incremental exercise test at different days.

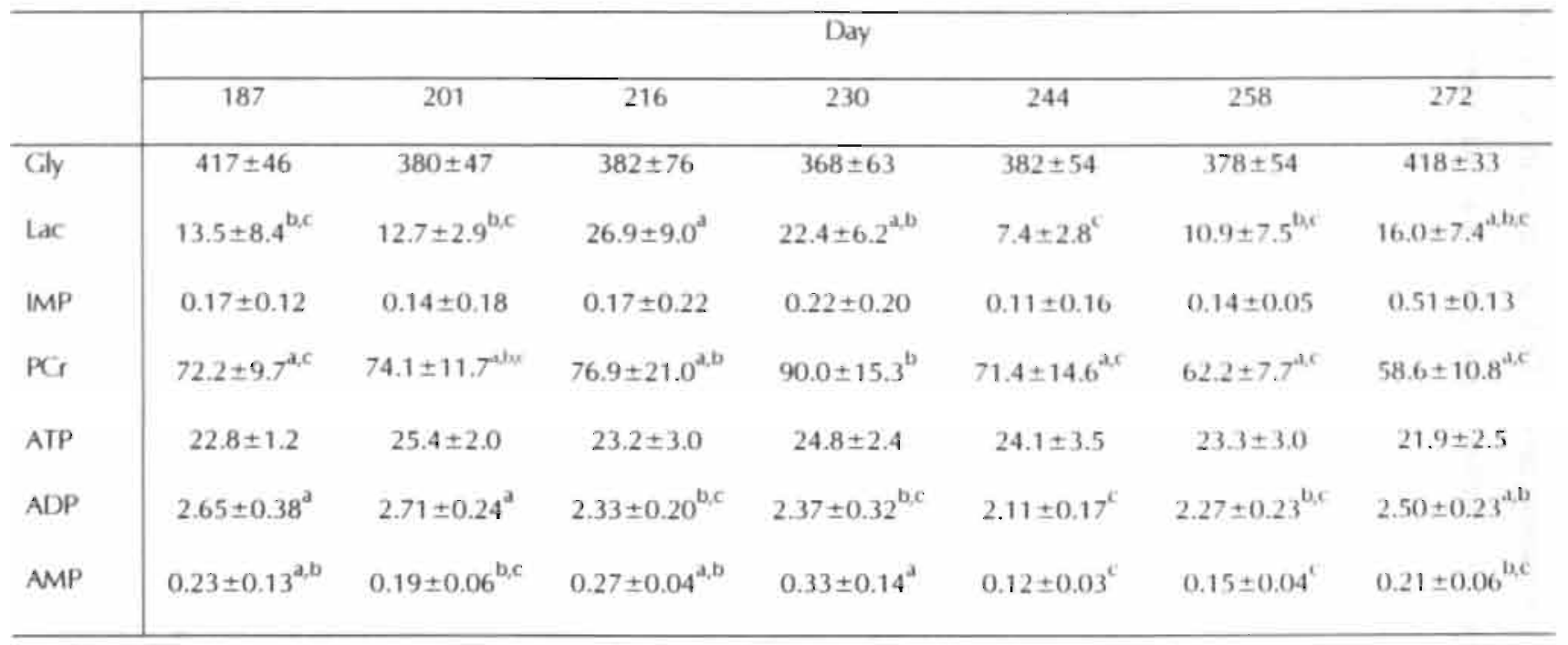

Values are means $\pm S f$ in mmolig dry $w ; n=7$ horses. Cly, glycogen; Lac, lactate; $P C r$, phosphocreatine. Significant differenas $(P<0.05)$, are indicated by differing superscripts. 
Table 5,3. Difference in muscle content of Gly, Lac; IMP, PG, ATP, ADP, and AMP before and immediately after increment.al excerise test at different days.

\begin{tabular}{|c|c|c|c|c|c|c|c|}
\hline & & & & Day & & & \\
\hline & 187 & 201 & 216 & 230 & 244 & 258 & 272 \\
\hline Gly & $93 \pm 33$ & $133 \pm 72$ & $121 \pm 92$ & $92 \pm 71$ & $157 \pm 82$ & $116 \pm 59$ & $108 \pm 55$ \\
\hline Lac & $-53 \pm 24.1^{b, c}$ & $-49 \pm 30.1^{c}$ & $-65 \pm 14.6^{a_{1} b_{1} c}$ & $-77 \pm 43.1^{a, b}$ & $-81 \pm 22.0^{a, b}$ & $-88 \pm 31.1^{4}$ & $-72 \pm 26,0^{a, b, c}$ \\
\hline IMP & $-0.41 \pm 0.77^{\mathrm{b}}$ & $-0.84 \pm 1.05^{a, b}$ & $-0.79 \pm 0.79^{a, b}$ & $-1.51 \pm 1.30^{a, b}$ & $-1.78 \pm 1.31^{4}$ & $-1.57 \pm 1.08^{-16}$ & $-1.24 \pm 1.36^{4.16}$ \\
\hline PCr & $28.5 \pm 12.1^{b}$ & $35.9 \pm 21.8^{\mathrm{a}, \mathrm{b}}$ & $49.0 \pm 21.6^{a}$ & $55.6 \pm 11.6^{a}$ & $41.8 \pm 17.1^{a, b}$ & $35.8 \pm 9.4^{.1 .17}$ & $37.9 \pm 6.8^{4.6}$ \\
\hline ATP & $-0.49 \pm 4.2^{b}$ & $5.50 \pm 4.0^{a}$ & $5.33 \pm 5.8^{\mathrm{a}}$ & $7.90 \pm 2.3^{a}$ & $8.01 \pm 4.4^{3}$ & $5.62 \pm 2.3^{4}$ & $2.62 \pm 2.4^{b}$ \\
\hline ADP & $0.05 \pm 0.37$ & $0.14 \pm 0.22$ & $-0.31 \pm 0.40$ & $0.25 \pm 0.43$ & $-0.14 \pm 0.16$ & $0.03 \pm 0.32$ & $-0.02 \pm 0.33$ \\
\hline $\mathrm{AMP}$ & $0.05 \pm 0.15$ & $0.01 \pm 0.15$ & $0.03 \pm 0.07$ & $0.04 \pm 0.08$ & $-0.06 \pm 0.16$ & $-0.02 \pm 0.05$ & $-10.00 \pm 0.06$ \\
\hline
\end{tabular}

Values are means \pm SE in mmolig dry $w t ; n=7$ horses. Significant differences $(P<0.05)$ are indicated by differing superseripts. 
In general the decline in muscle glycogen was on the order of $100-150 \mu \mathrm{mol}$ glucose moieties/gram dry tissue $w t$, corresponding to a $\sim 25 \%$ decrease in endogenous muscle glycogen. There was no significant difference between the tests performed at the various exercise test days. Also, during the period of staleness (from day 258), no effect on resting and postexercise glycogen content was observed. The muscle content of lactate before the incremental exercise test varied considerably. Mean values from 7.4 to 26.9 $\mu \mathrm{mol} / \mathrm{g}$ dry $\mathrm{wt}$ were observed. These differences were rather inconsistent, and no relationship between the pattern observed and the phase of the experiment or occurrence of overtraining could be detected. Tissue lactate levels were significantly increased after the incremental exercise test. The increase varied from 49 to $88 \mu \mathrm{mol}$ lactate/g dry wt. The lactate levels during the period of overtraining were in the same ranges as measured on previous days.

The pre-test ATP levels did not change throughout the entire experimental period (Table 5.2). A significant fall in tissue ATP was observed immediately after the exercise test (Table 5.3). The decline in tissue ATP during the exercise test was significantly less when signs of overtraining were present. Tissue levels of ADP and AMP showed slight but significant variations in the pre-test biopsies throughout the experimental period. No consistent changes were observed after the exercise test. The tissue levels of IMP, a degradation product of adenine nucleotides, were on the order of 0.11 to $0.51 \mu \mathrm{mol} / \mathrm{g}$ dry tissue $w t$ in the pre-test sample throughout the experimental period $(P>0.05)$. After the incremental exercise test, the tissue IMP content was found to be significantly increased. Because of a substantial interindividual variability, no significantly consistent pattern could be observed throughout the study.

$\mathrm{PCr}$ levels in the biopsy samples taken before the exercise test showed a steady and significant decline from day 230 to day 272 . After exercise a significant decrease in $\mathrm{PCr}$ was found. This decline was not significantly different between exercise tests. The extent of $\mathrm{PCr}$ depletion was not related to the phase of the training or the onset of overtraining (Table 5.3). 


\subsubsection{Adrenal response to $\mathrm{ACTH}$}

Resting cortisol levels differed significantly during the study, whereas no. relationship was observed with symptoms of overreaching or staleness (Table 5.4, time 0 h). Two hours after ACTH injection at day 271, when the animals showed symptoms of overtraining, a significantly higher cortisol level was reached than during the three previous tests. Four hours after ACTH injection, no difference in plasma cortisol levels was observed. Peak cortisol levels were obviously reached before $4 \mathrm{~h}$ after injection of ACTH.

\subsubsection{Blood chemical variables}

Plasma urea and creatinine concentrations failed to show any systematic changes throughout the training period. The values remained within the range that is considered as normal (data not shown).

The plasma activity of CK was not significantly elevated before or during overtraining (data not shown). Occasionally levels of $>200 \mathrm{U} / \mathrm{I}$ with a maximum of 1,000 U/l were observed. No relationship among plasma CK activity, the training program on the previous days, and symptoms of overtraining could be detected. Prior to and during the period of overt signs of staleness or overtraining syndrome, the mean plasma CK activity did not consistently increase.

\subsection{DISCUSSION}

The purpose of the present investigation was to study the adaptation and performance capacity to a training program, consisting of exercise gradually increasing in volume and intensity, and to search for blood chemical and physiological markers of overtraining. 
Iable 5.4 Plasma cortisol levels at different days before and 2 and $4 \mathrm{~h}$ after $\mathrm{ACTH}$ injection

\begin{tabular}{|c|c|c|c|c|c|}
\hline \multirow[b]{2}{*}{ Time } & \multicolumn{5}{|c|}{ Day } \\
\hline & 200 & 229 & 243 & 257 & 271 \\
\hline oh & $0.15 \pm 0,06^{d}$ & $0.19 \pm 0.06^{4}$ & $0.22 \pm 0.07^{10}$ & $0.19 \pm 0.04^{2.1}$ & $0.20 \pm 0.04^{2.11}$ \\
\hline $2 \mathrm{~h}$ & $0.47 \pm 0.13^{4}$ & $0.33 \pm 0.05^{b}$ & $0.38 \pm 0.11^{\mathrm{t}}$ & $0.33 \pm 0.06^{\prime \prime}$ & $0.43 \pm 0.06^{\prime \prime}$ \\
\hline $4 \mathrm{~h}$ & $0.39 \pm 0.14^{d}$ & $0.26 \pm 0.10^{b}$ & $0.28 \pm 0.09^{\prime \prime}$ & $0.22 \pm 0.05^{13}$ & $0.25 \pm 0.06^{1}$ \\
\hline
\end{tabular}

Values are means in mmol/ $; n=7$ horses. Significant differences $(P<0.05)$ within test-days are indicated by differing superscripts. 
The present study demonstrates that a progressive increase in the training loads could be tolerated by the horses as long as days of intensive exercise were alternated with days of endurance running at moderate intensity (v140).

As soon as the intensity of the endurance running was increased to v180, the animals demonstrated difficulties in sustaining the intensive exercise bouts, and an increasing number of animals were unable to complete the intensive training. The inability to complete the training is considered to reflect overtraining. Aiter this first sign of overtraining, 2-3 days later the animals were not only unable to complete the interval training but also showed signs of irritability and sluggishness and did not eat the normal amount of food offered. The first phase of overtraining reflected in the inability to complete intensive training is considered to reflect early overtraining or overreaching (5, 9). After 1-2 days, in addition to the inability to complete the training sessions, the animals showed a complex of symptoms suggestive for overtraining syndrome or staleness $(5,9)$. However, because no additional testing was done, it remains unknown whether the overtraining in the present study concerned staleness or overreaching. It is assumed that alternating high-intensity exercise with low-intensity exercise allows the organism to recover and to adjust to the physical stress (9). However, by the intensity of the endurance exercise being increased from v 140 to v180, recovery was most likely compromised, resulting in incomplete restoration of homeostasis at the onset of each training session.

Despite the fact that the horses showed symptoms of overtraining from day 263 , this was not reflected in a significant decline of performance during the exercise test. However, the horses were not able to complete the training sessions because of premature fatigue. It cannot be ruled out that small changes in performance capacity as a consequence of overtraining remained unnoticed because they stayed within the normal variability. Observations in human athletes demonstrate that the normal variability in maximal work load on a cycle ergometer is $\sim 5 \%$ (8), and that in overtrained cyclists the maximal work load is declined between 5 and $10 \%$ (6). Therefore, the treadmill test as 
Chapter 5

used in the present study may not have been sensitive enough to detect small changes in performance (8).

One of the significant findings of the present study is that, other than the inability to complete the training because of premature fatigue, no other characteristic warning signs for overtraining were found. Premature fatigue during the training was followed by changes in behaviour, such as increased irritability and reluctance to exercise. This is in agreement with the findings in human athletes who are subjected to a strenuous training protocol (11). Blood chemical changes that have been associated with overtraining in humans are increases in plasma urea concentration and plasma activity of muscle enzymes (7). Plasma urea concentration never exceeded the upper levels of the normal range. This indicates that overtraining in the horse is not associated with an increased breakdown of protein. In addition, because the plasma CK activity did not increase during overtraining, overall sarcolemmal integrity is suggested to be maintained during overreaching and staleness. Hence, the results of the present study demonstrate that plasma urea concentration and plasma CK activity are poor markers for an imbalance between training and recovery. This is in line with findings reported by others (12). This is also in accordance with histological examination of the biopsy samples from the middle gluteal muscle, which failed to show signs of inflammation or degeneration. This indicated that overtraining is not necessarily associated with muscle damage.

Resting cortisol levels failed to change significantly during overtraining. Conflicting results have been reported about resting cortisol levels in overtrained athletes. Barron et al. (1) reported decreased resting cortisol levels in overtrained human athletes, whereas elevated resting cortisol levels have been reported in overtrained swimmers (13). It is possible that the resting cortisol levels depend on the duration of the overtraining, i.e., increased plasma cortisol levels occur with early overtraining, whereas prolonged overtraining results in decreased cortisol levels (18).

The adrenocortical response to $\mathrm{ACTH}$ during overtraining was increased compared with previous tests, suggesting an increased responsiveness. This would contradict the findings as reported by Persson et al. (16), who observed a decreased 
cortisol response after intramuscular $\mathrm{ACTH}$ injection in overtrained horses. Persson et al. (16), however, studied animals with severe and progressed staleness, whereas in the present study most likely early overtraining was encountered. Therefore, it cannot be ruled out that prolonged overtraining is associated with decreased adrenal responsiveness.

The red blood cell volume was not changed throughout the study. This is in contrast with the findings as reported by Persson et al. (16), who observed an increased red blood cell volume in overtrained horses. However, as mentioned above, Persson and colleagues (16) studied horses with progressed staleness. It cannot be ruled out that progressed staleness is associated with an increase in red blood cell volume.

The data from the present study șow that muscle glycogen levels were normal throughout the study and were not decreased during overreaching and staleness. Although Costill et al. (2) reported that insufficient intake of carbohydrate and decreased glycogen levels are important factors for inducing overtraining, the present study shows that overtraining can occur despite normal resting muscle glycogen levels. The data from the present study suggest that insufficient time for recovery is important rather than inadequate carbohydrate intake and insufficient repletion of muscle glycogen. The normal resting glycogen levels may explain the normal plasma lactate levels during the exercise tests. Some authors have reported that lower plasma lactate levels during given work loads can be observed in overtrained athletes, as well as lower maximal plasma lactate levels $(2,4,6)$. This is generally attributed to a lower muscle glycogen pool at the start of the exercise bout, although Lehmann et al. (10) attributed lower lactate levels to decreased catecholamine output during overtraining. Lehmann et al. (10), however, did not actually measure muscle glycogen levels.

The inability to complete the intensive training because of fatigue cannot easily be explained by the biochemical data obtained from the muscle samples before and immediately after the exercise test. Resting muscle glycogen levels and glycogen use during the exercise test did not change throughout the study. Thus the early fatigue cannot be attributed to premature glycogen depletion. The drop in tissue ATP during the 
incremental exercise test was significantly less during the last test when the animals showed symptoms of overtraining. Interestingly, the decreased drop of ATP was not reflected in the larger decline in PCr or higher increases in lactate. Taking into account that the same amount of work was performed during the exercise test, the present findings suggest an improvement of ATP regeneration during exercise after the onset of overtraining. This conclusion is, however, rather speculative and further experimentation is required to explore the relationship between overtraining and ATP utilisation.

In conclusion, the present study demonstrates that overtraining can be induced in horses by gradually increasing the training loads. The earliest sign of overtraining is the inability to complete the intensive training, with associated increased irritability and reluctance to exercise. The generally advocated blood chemical variables failed to demonstrate specific changes during overtraining in the horse.

\subsection{REFERENCES}

1. Barron, G.L., T.D. Noakes, W. Levy, C. Smith, and R.P. Millar. Hypothalamic dysfunction in overtrained athletes. J Clin Endocrinol Metab 60: 803-806, 1985.

2. Costill, D.L., M.G. Flynn, J.P. Kirwan, J.A. Houmard, J.B. Mitchell, R. Thomas, and S.H. Park. Effects of repeated days of intensified training on muscle glycogen and swimming performance. Med Sci Sports Exercise 20: 249-254, 1988

3. Dubowitz, V. Muscle Biopsy, a Practical Approach (2nd ed). London: Baillere Tindall, 1985, p.29.

4. Foster, C., A.C. Snyder, N.N. Thompson, and K. Kuettel. Normalization of the blood lactate profile in athletes. Int J Sports Med 9: 198-200, 1988.

5. Fry, R.W., R. Morton, and D. Keast. Overtraining in athletes; an update. Sports Med 12: $32-65,1991$.

6. Jeukendrup, A.E., M.C.K. Hesselink, A.C. Snyder, H. Kuipers, and H.A. Keizer. Physiological changes in male competitive cyclists after two weeks of intensified training. Int I Sports Med 13: 534-541, 1992 
7. Kindermann, W. Das Übertraining - Ausdruck einer vegetativen Fehlsteuerung. Disch Z Sportmed 8: 138-145, 1986.

8. Kuipers, H., F. Verstappen, H. Keizer, P. Geurten, and G.van Kranenburg. Variability of aerobic performance in the laboratory and its physiological correlates. In .J Sports Med 6: 197-201, 1985.

9. Kuipers, H., and H.A. Keizer. Overtraining in elite athletes: review and directions for the future. J Sports Med 6:79-92, 1988.

10. Lehmann, M., P. Baumgartl, C. Wiesenack, A. Seidel, H. Baumann, S. Fischer, U. Spüri, G. Gendrisch, R. Kaminiski, and J. Keul. Training-overtraining: influence of a defined increase in training volume vs. training intensity on performance, catecholamines and some metabolic parameters in experienced middle and long distance runners. Eur J Appl Physiol Occup Physiol 64: 169-177, 1992.

11. Morgan, W.P., D.R. Brown, J.S. Raglin, J. O'Connor, and K.A. Ellickson. Psychological monitoring of overtraining and'staleness. Br J Sports Med 21: 107-114, 1987.

12. Nosaka, K., P.M. Clarkson. Relationship between post-exercise plasma CK elevation and muscle mass involved in the exercise. Int J Sports Med 13: 471-475, 1992.

13. O'Connor, P.J., W.P. Morgan, J.S. Raglin, C.M. Barksdale, and N.H. Kalin. Mood state and salivary cortisol levels following overtraining in female swimmers. Psychoneuroendocrinology 14: 303-310, 1989.

14. Passoneau, J.V., Lactate. Fluorometric method. In: Methods of Enzymatic Analysis. Edited by H.U. Bergmeyer. New York: Academic Press, 1974, pp. 1468-1472.

15. Persson, S.G.B. On blood volume and working capacity in horses. Acta Vet Scand Suppli 19: $1-189,196 ?$

16. Persson, S.C.B., M. Larsson, and A. Lindholm. Effects of training on adrenal-cortical function and red-cell volume in trotters. Zentralb! Vet Med Reihe A 27: 261-268, 1980.

17. Snow, D.H., and R.C. Harris. Limitations to maximal performance in the racing thoroughbred. In: Biochemistry of exercise VI, edited by B. Saltin. Champaign, IL: Human Kinetics, 1986, pp. 447-454.

18. Stone, M.H., R.E. Keith, I.T. Kearney, S.J. Fleck, G.D. Wilson, and N.T. Triplett. Overtraining: a review of the signs, symptoms and possible causes. I Appl Sport Science Res 5: 35-50, 1991. 


\section{Chapter 5}

19. Van der Vusse, G.J., W.A. Coumans, F.H. van der Veen, A. Drake, W. Flameng, and R. Suy. ATP, creatine phosphate and glycogen content in human myocardial biopsies: markers for the efficacy of cardioprotection. Vasc Surg 18: 127-134, 1984.

20. Van der Vusse, G.J., G.M.E. Janssen, W.A. Coumans, H. Kuipers, R.J.M.M. Does, and F. ten Hoor. Effect of training and 15, 25, and $42 \mathrm{~km}$ contests on skeletal muscle content of adenine and guanine nucleotides, creatine phosphate, and glycogen. Int I Sports Med 10 Suppl 3: S146-S152, 1989.

21. Viru, A. The mechanism of training effects: a hypothesis. Int J Sports Med 5: 219-227, 1984.

22. Wijnants, J., and H. van Belle. Single-run high performance liquid chromatography of nucleotides, nucleosides and major purine bases and its application to different tissue extracts. Anal Biochem 144: 258-266, 1985. 
Chapter 6

\section{EFFECTS OF 3-MIN SUBMAXIMAL. EXERCISE BOUTS COMBINED WITH ENDURANCE} TRAINING ON THE PERFORMANCE CAPACITY OF HORSES

This chapter will be published as:

G. Bruin, H. Kuipers, G. André, and G.J. van der Vusse

Effects of 3-min submaximal exercise bouts combined with endurance training on the performance capacity of horses Int J Sports. Med (Accepted for publication) 


\subsection{ABSTRACT}

In a randomised block design, 24 Standardbred trotter stallions were used to investigate the effect of the number and intensity of 3-minute aerobic work bouts on the performance capacity. Performance capacity was expressed as the regression of heart rate versus power output, plasma lactate versus power output, the total running time, and the total amount of vertical work. In addition, plasma volume, red blood cell volume, and skeletal muscle levels of glycogen, lactate, energy-rich phosphates and related compounds were determined. The horses were trained three times a week during a period of 104 days. The exercise protocol consisted of either 3 or 6 intervals at $70 \%$ or $85 \%$ of the peak heart rate, interspersed by 3-min exercise at an intensity of $60 \%$ of the peak heart rate. Prior to the experiment, and at day 54 and 106, the horses performed a stepwise increasing exercise test until exhaustion on a treadmill

Aerobic exercise with an intensity of $70 \%$ or $85 \%$ of the peak heart rate, resulted in an increase of performance capacity of the horses, in all' four training regimens, when the amount of vertical work delivered on the treadmill was considered (up to $14 \%$, irrespective of the training protocol). The improvement of total running time until exhaustion was less consistent. Red blood cell volume and plasma volume increased with a faster rise in the groups with the higher exercise intensity. During the experimental period an increase of muscle glycogen and AMP, and a decrease of resting ATP was observed. Resting phosphocreatine levels remained unchanged.

Immediately after the incremental exercise test a decrease of muscle content of glycogen, phosphocreatine and ATP of about 30-40\%, 50\% and $20-30 \%$ respectively, was found. Muscle lactate and' IMP' levels showed an increase up to 75-100 and 2-3 $\mu$ mol/g dry weight, respectively. No differences were observed between the training. protocols and the days of the test. No changes were seen in the relationship between 
heart rate or plasma lactate and power output. The different exercise protocols provided comparable results.

In summary, the data of the present study demonstrate that in horses an aerobic training protocol, consisting of 3 or 6 times 3 min work bouts at intensities of either $70 \%$ or $85 \%$ of the peak heart rate, results in an improved performance capacity of about $10 \%$. This improvement was irrespective of the intensity and/or number of the work bouts. Because of the effect of exercise intensity on the increase in red blood cell volume, it is advised to use an intensity of $85 \%$ of the maximal heart rate. The number of repetitions should be kept as low as possible to prevent injuries. Three repetitions as used in this study proved to be sufficient for the increase in oxygen transport capacity. 


\subsection{INTRODUCTION}

One of the important determining factors for physical adaptation to training is the intensity of exercise $(2,4,16,24,26,28,34)$. In order to achieve the maximal improvement of performance capacity in an individual, the optimal frequency, exercise intensity, and duration of exercise have to be known. In humans, an exercise intensity of $70 \%$ of the maximal heart rate appears to be the minimal training stimulus to provide improvements of the aerobic capacity. A lower intensity can be compensated by a longer duration of the exercise bout imposed on the athlete or a higher training frequency (16). In experiments with interval training in humans, in which the effect of number of training sessions was studied, it was found that the effect on $\mathrm{VO}_{2 \max }$ of 2 versus 5 training sessions weekly, was comparable (16). Well controlled experimental data on the effect of training on horses are relatively scarce. In practical horse training, usually days of interval training are alternated by days on which endurance exercise at moderate intensity is used. It is largely unknown, however, which number of repetitions and which exercise intensity yield the largest effects on aerobic performance capacity, and whether the training effect is linearly related to the number of exercise bouts.

Therefore, the purpose of the present study was to investigate in horses the effects of the number of repetitions as well as the exercise intensity on the performance capacity with exercise of submaximal intensity. To this end the effect of three or six $3-\mathrm{min}$ bouts of exercise at $70 \%$ or $85 \%$ of the peak heart rate on performance capacity, measured on a treadmill, were studied. To obtain insight into the physiological basis of possible changes in performance capacity a number of physiological and biochemical variables was measured, such as plasma volume, red blood cell volume and, in muscle biopsies, glycogen, lactate, energy-rich phosphates and related compounds. 


\subsection{MATERIAL AND METHODS}

\subsubsection{Horses and training}

Twenty-four 2-6 year old Standardbred trotter stallions entered the study that was spread over a two year period for practical reasons. During two consecutive years 12 different horses participated and were subjected to the same training regimen. In a randomised block design the horses were assigned to one of four different treatments. Heart rate was used to determine the exercise intensity. The exercise intensity was based on fixed percentages of the peak heart rate. The peak heart rate was the maximal heart rate ever measured in the individual horse under investigation during an exercise test until exhaustion.

The four different training protocol treatments consisted of:

group 1: $\quad 3$ bouts of $3 \mathrm{~min}$ at $70 \%$ interspersed with $3 \mathrm{~min}$ at $60 \%$ of the peak heart rate group 2: $\quad 3$ bouts of 3 min at $85 \%$ interspersed with 3 min at $60 \%$ of the peak heart rate group 3: $\quad 6$ bouts of $3 \mathrm{~min}$ at $70 \%$ interspersed with $3 \mathrm{~min}$ at $60 \%$ of the peak heart rate group 4: $\quad 6$ bouts of $3 \mathrm{~min}$ at $85 \%$ interspersed with $3 \mathrm{~min}$ at $60 \%$ of the peak heart rate.

Each training session was preceded by a 6 min warming-up at $4.5 \mathrm{~m} / \mathrm{s}$. The four different training protocols were performed on a treadmill with an inclination of $6.25 \%, 3$ times a week for a period of 106 days. The days with treadmill training were interspersed with days on which endurance running at an intensity lower than $60 \%$ of the peak heart rate was performed in a rotary exerciser. In the afternoon of the days with treadmill exercise the horses. performed an exercise bout of $30 \mathrm{~min}$ at $1.5 \mathrm{~m} / \mathrm{s}$ in a rotary exerciser. In total the horses were exercised for 6 days per week with 1 day complete rest. Once a week the speed of the treadmill during the interval bouts and endurance exercise was adjusted according to the results of training and tests in order to obtain the desired level of 70 or $85 \%$ of the peak heart rate. The horses were individually housed, and fed according to the energy requirements. 


\subsubsection{Working procedure}

Every day at 8 a.m. the weight of the horses was measured.

On test days, after weighing, the right jugular vein was catheterised and after anaesthetisation of the skin a biopsy was taken from the gluteus medius muscle $(3,6)$. The safety belt and the belt for the heart rate monitor were attached. The ecgequipment was also attached to the belt of the heart rate monitor. Blood samples were taken prior to exercise, during the last $15 \mathrm{~s}$ of each step, 2 and $5 \mathrm{~min}$ after finishing the test and immediately after every training session.

Immediately after the test $15 \mathrm{ml} 2 \%$ Evan's Blue dye was injected (thereafter the catheter was washed with $5 \mathrm{ml}$ physiologic saline solution), a muscle biopsy was taken and the rectal temperature was measured. After that, the horse was showered, weighed and put into the housing box. Fifteen min after the Evan's Blue injection a blood sample was taken to determine the plasma volume. The tests were performed between 7 a.m. and noon.

\subsubsection{Incremental exercise test and data collection}

During the experimental period, three incremental exercise tests were done on the treadmill (day 0, day 54 and day 106). The incremental exercise tests were preceded by one day rest. After a $6 \mathrm{~min}$ warming-up at $4.5 \mathrm{~m} / \mathrm{s}$, the incremental exercise tests consisted of a 2 min step at a treadmill speed of $2 \mathrm{~m} / \mathrm{s}$. Thereafter the speed was increased to $5 \mathrm{~m} / \mathrm{s}$ and every 2 min further increased with $1 \mathrm{~m} / \mathrm{s}$ until exhaustion. Exhaustion was considered to be reached when, despite encouragement, the horse was not able to maintain the running speed.

During training exercise and during the incremental exercise test the heart rate was continuously recorded every 5 s by means of the "Sport tester PE3000" (Polar Electro) and during the incremental exercise tests also by means of an electrocardiogram (ecg) (Schwarzer CS6000). During the incremental exercise test time to exhaustion was measured. Since the treadmill had a $6.25 \%$ incline, the power 
output and the total amount of vertical work ( $\mathrm{kJ}$ ) delivered until exhaustion could be calculated.

The power output (Watt) is calculated according to the formula:

$$
\text { power }=9.81 \times \text { body weight } \times \text { speed } \times \sin \alpha
$$

In this formula body-weight is expressed in $\mathrm{kg}$, speed in $\mathrm{m} / \mathrm{s}$, and $\alpha$ is the angle (degrees) of inclination of the treadmill.

The amount of vertical work (kJ) per stage is calculated according to the formula:

$$
\text { work }=9.81 \times \text { body-weight } \mathrm{x} \text { speed } \mathrm{x} \text { duration } \mathrm{x} \sin \alpha
$$

in which duration is the running time (s) at each treadmill speed. The amount of work at each speed is summated, resulting in the total amount of work. Only the vertical component was included.

The relationship between heart rate and power output is described by the linear relation $(H R=$ heart rate in beats $/ \mathrm{min} ;$ power $=$ power output in Watt; $\beta$ ${ }_{0}=$ intercept;

$\beta_{1}=$ regression coefficient:

$$
H R=\beta_{0}+\beta_{1} \times \text { power }
$$

The relationship between plasma lactate concentration $(\mathrm{mM})$ and power output (Watt) is described by the exponential curve:

$$
\text { lactate }=A+B \times R^{\text {power }}
$$

in which lactate is the plasma lactate concentration $(\mathrm{mM}), \mathrm{A}, \mathrm{B}$ and $\mathrm{R}$ are regression parameters, and power is the power output (Watt).

Changes in the performance capacity were measured as changes in the regression of heart rate (beats/min) versus power output (Watt), plasma lactate (mM) versus power output (Watt), the total running time until exhaustion (s), and the total amount of vertical work until exhaustion ( $k J)$. 


\subsubsection{Measurement of plasma lactate and muscle enzymes}

The samples were centrifuged in heparinized Eppendorf-cups at 12,000 revolutions per min $(8800 \mathrm{~g})$. The plasma was immediately frozen at $-20^{\circ} \mathrm{C}$ and subsequently stored at $-80^{\circ} \mathrm{C}$. Plasma lactate was determined with a Lactate analyser 640 (La Roche). The plasma activity of creatine kinase (CK), aspartate aminotransferase (AST), alanine aminotransferase (ALT) and lactate dehydrogenase (LDH) was determined by means of enzymatic spectrophotometry (Boehringer*).

\subsubsection{Measurement of plasma volume and red blood cell volume}

The blood sample was centrifuged during $10 \mathrm{~min}$ at 2,000 revolutions per $\mathrm{min}$. The resulting plasma was centrifuged again during $30 \mathrm{~min}$ at 12,000 revolutions per min and frozen at $-20^{\circ} \mathrm{C}$. The frozen samples were stored at $-80^{\circ} \mathrm{C}$. The plasma volume was calculated according to:

$$
P V=\frac{E B}{C}
$$

where PV is the plasma volume in $\mathrm{ml}, \mathrm{EB}$ the amount of injected Evan's blue dye in $\mathrm{mg}$, and $\mathrm{C}$ the concentration of the dye in $\mathrm{mg} / \mathrm{ml}$ plasma.

The normalised red blood cell volume is:

$$
C V=\frac{P V \times H c t}{(100-H c t) \times b o d y w e i g h t}
$$

where CV is the red blood cell volume in $\mathrm{ml} / \mathrm{kg}_{\text {, }} \mathrm{PV}$ the plasma volume in $\mathrm{ml}$ and $\mathrm{Hct}$ the venous hematocrit (\%). Body weight is expressed in $\mathrm{kg}$.

\subsubsection{Measurement of muscle levels of energy-rich phosphates, glycogen and lactate}

The metabolic status of the muscular tissue was judged by determination of the amount of phosphocreatine (PCr), adenosine triphosphate (ATP), adenosine diphosphate (ADP), adenosine monophosphate (AMP), and inosine monophosphate 
(IMP) in biopsies obtained from the gluteus medius muscle. Also the tissue contents of glycogen and lactate were determined.

The portion of the biopsy that was used for biochemical analysis was rapidly frozen in liquid nitrogen. Time interval between sampling of tissue and freezing was in the order of 5-10 s. The frozen tissue samples were stored at $-80^{\circ} \mathrm{C}$. Prior to biochemical analysis the samples were freeze-dried at $-30^{\circ} \mathrm{C}$ overnight.

Freeze-dried tissue was weighed and divided in at least two parts. One part ( $\sim 10 \mathrm{mg}$ dry wt was used for the determination of adenine nucleotides and related compounds, phosphocreatine, lactate, and glucose. To this end, the tissue sample was extracted at $-15^{\circ} \mathrm{C}$ in a mixture of perchloric acid $(3 \mathrm{M})$ and dithiothreitol $(5 \mathrm{mM})$. The extraction volume was adjusted to the dry weight of the tissue specimen $(\sim 25 \mu \mathrm{l}$ extraction fluid per mg dry tissue weight). The tissue was ground with a glass rod in the extraction fluid and subsequently rapidly frozen in liquid nitrogen and thawed to a temperature of $4^{\circ} \mathrm{C}$. The mixture was centrifuged at $4^{\circ} \mathrm{C}$ with $1200 \mathrm{~g}$ for $5 \mathrm{~min}$. An aliquot of the supernatant was removed and neutralised with $\mathrm{KHCO}_{3}$ (36). The neutralised supernatant was stored at $-80^{\circ} \mathrm{C}$ until further analysis.

The second part of the freeze-dried tissue ( $\sim 5 \mathrm{mg}$ dry wt) was used for glycogen determination. The tissue specimens were kept at $100^{\circ} \mathrm{C}$ for $3 \mathrm{~h}$ after addition of $1.0 \mathrm{ml}$ of $1 \mathrm{M} \mathrm{HCl}$. Thereafter, the samples were neutralised with tris(hydroxymethyl)-aminomethane $(0.12 \mathrm{M})-\mathrm{KOH}(2.1 \mathrm{M})$, saturated with $\mathrm{KCl}$. The glucose residues were measured fluorometrically as described elsewhere (35). The values obtained were corrected for the amount of free glucose already present in the tissue at the time of sampling.

The tissue content of adenine nucleotides and related compounds was determined by high-performance liquid chromatography with the use of a method modified after Wijinants and Van Belle (37) and described by Van der Vusse et al. (36). Briefly, a small sample of the neutralised extract $(10 \mu \mathrm{l})$ was applied to a reverse phase column 
(Lichrosorb RP-18, Merck). Stepwise gradient elution, using two solvents, was applied to separate the compounds of interest. Flow rate amounted to $0.8 \mathrm{ml} \cdot \mathrm{min}^{-1}$. Solvent A was an aqueous buffer of $\mathrm{NH}_{4}\left(\mathrm{H}_{2} \mathrm{PO}_{4}\right)(150 \mathrm{mM}$, pH $=6.0)$, solvent $\mathrm{B}$ consisted of a 1:1 (vol/vol) mixture of acetonitrile and methanol. Peaks were detected at $254 \mathrm{~nm}$ and identified by comparing retention times with known standards. In case of IMP, the presence of this substance in the extraction fluid was also positively identified by comparing the extinction of the peak at two different wave lengths (i.e., 254 and 293 $\mathrm{nm})$. LiChroCart 4-4 (Merck) was used as guard column.

$\mathrm{PCr}$ was measured fluorometrically as described earlier (35). Lactate was assayed according to Passoneau (21). Free glucose was assayed as described (35) and expressed as micromoles per gram dry weight.

\subsubsection{Statistics}

The experiments were performed in a completely randomised block design. Blocks were based on performance capacity immediately before the start of the experiment. In a single experiment 12 horses were used. The two experiments were identical and the data were analysed together using the Genstat package (9).

Analysis was performed according to the following model:

$$
y_{i j k}=\mu+\beta_{i}+\alpha_{j}+\varepsilon_{i, j}+\tau_{k}+(\alpha \tau)_{j k}+\varepsilon_{2, j k}
$$

with:

$$
\begin{array}{ll}
y_{i, k} & \text { response horse } j \text { in block } i \text { in test } k \\
\mu & \text { level } \\
B_{1} & \text { random effect block } i(i=1 \ldots 3), B \sim N\left(0, \sigma_{b}{ }^{2}\right) \\
\alpha_{i} & \text { fixed effect treatment } j(j=1 \ldots 4) \\
\varepsilon_{1, j} & \text { rest }: \text { variance between horses within block, } \varepsilon_{i} \sim N\left(0, \sigma_{p}{ }^{2}\right) \\
\tau_{k} & \text { fixed effect test } k(k=1 \ldots n) n=(\text { max }) \text { number of tests. } \\
(\alpha \tau)_{j,} & \text { interaction effect treatment } j \text { in test } k \\
\varepsilon_{2, j k} & \text { rest }{ }_{2}: \text { variance between tests within horses, } \varepsilon_{2} \sim N\left(0, \sigma_{e}{ }^{2}\right)
\end{array}
$$


For reasons of readability, neither the year-effect for the two combined experiments nor the notation for the orthogonal polynomes is included in the formula. Treatment effect is presented with only one symbol (j). In reality more factors were involved (in a factorial design). Time course was simultaneously tested with the F-test, while conditionally, if the F-test produced a significant effect, Students' t-test was performed for pairs. The different populations were compared with the pooled estimate of the variance of difference. The standard error of difference of means (sed), multiplied by Students' $t_{v}$, produces the least significant difference (Isd). The value of $t_{v}$ is $\sim 2$, depending of the degrees of freedom. Differences were regarded significant at $p$ $<0.05$.

Before combining the data of the two identical experiments, homogeneity of variances was analysed according to Snedecor et al. (30). Interactions between experiment and treatment were analysed as well. The heterogeneity between horses proved to be large and blocks did not reduce the experimental error.

\subsection{RESULTS}

\subsubsection{Acceptance of the exercise protocol and training intensity}

The horses tolerated the exercise protocol very well. In total 16 out of 912 planned training sessions were skipped. None of the horses missed more than 2 sessions. No serious health problems were observed during the experimental period. The blood plasma levels of muscle enzymes (CK, AST, LDH) were found to be within normal ranges and were without systematic changes (data not shown). The same holds for the liver enzyme ALT.

Analysis of the heart rate recordings during training revealed that the horses in the groups with 3 or 6 intervals at nominally $70 \%$ of the peak heart rate had exercised at an average of $73 \%$ and $72 \%$ of the peak heart rate, respectively. The groups with 3 
or 6 intervals at nominally $85 \%$ of the peak heart rate had exercised at an average of 87 and $86 \%$ of the peak heart rate, respectively.

Plasma lactate levels, at the end of the interval training, showed an average of $1.0 \pm 0.3$ and $1.5 \pm 1.1 \mathrm{mM}$ in the groups with 3 or 6 intervals at $70 \%$ of the peak heart rate, respectively, and an average of $2.7 \pm 1.3$ and $3.3 \pm 1.9 \mathrm{mM}$ in the groups with 3 or 6 intervals at $85 \%$ of the peak heart rate, respectively.

\subsubsection{Incremental exercise test, plasma lactate, plasma volume and red blood cell} volume.

Table 6.1 and 6.2 show the findings of running time until exhaustion and the amount of vertical work of the various regimens in the incremental exercise tests on day 0,54 , and 106 .

Table 6.1 Running time until exhaustion (s) during the incremental exercise test in the different training protocols.

\begin{tabular}{|c|c|c|c|}
\hline \multirow[b]{2}{*}{ Training protocol } & \multicolumn{3}{|c|}{ Day of experimental period } \\
\hline & 0 & 54 & 106 \\
\hline $3 \times 3 \mathrm{~min}$ at $70 \%$ & $741 \pm 61$ & $770 \pm 58$ & $778 \pm 60$ \\
\hline $3 \times 3 \min$ at $85 \%$ & $740^{\mathrm{a}} \pm 70$ & $815^{b} \pm 67$ & $781^{b} b_{ \pm 26}$ \\
\hline $6 \times 3 \mathrm{~min}$ at $70 \%$ & $748^{a} \pm 22$ & $786^{a b} \pm 39$ & $791^{b} b_{ \pm 4}$ \\
\hline $6 \times 3 \mathrm{~min}$ at $85 \%$ & $728^{a} \pm 45$ & $759^{a b_{ \pm 38}}$ & $776^{\mathrm{b}} \pm 81$ \\
\hline
\end{tabular}

Data are given as mean and standard deviation. Significant differences $(P<0.05)$ within one training protocol, are indicated in the horizontal direction by the superscripts $a, b, c$, meaning no difference in case of an identical letter $(n=24$, horses; standard error of differences $(s e d)=20$ ). 
Table 6.2 Amount of vertical work $(\mathrm{k} f)$ delivered during the incremental exercise test of the different training protocols.

\begin{tabular}{l|ccc}
\hline & \multicolumn{3}{|c}{ Day of experimental period } \\
\hline training protocol & 0 & 54 & 106 \\
\hline $3 \times 3 \mathrm{~min}$ at $70 \%$ & $1404^{\mathrm{a}} \pm 239$ & $1486^{\mathrm{a}} \mathrm{b}_{ \pm 232}$ & $1562^{\mathrm{b}} \pm 260$ \\
$3 \times 3 \mathrm{~min}$ at $85 \%$ & $1328^{\mathrm{a}} \pm 262$ & $1516^{\mathrm{b}} \pm 192$ & $1469^{\mathrm{b}} \pm 171$ \\
$6 \times 3 \mathrm{~min}$ at $70 \%$ & $1423^{\mathrm{a}} \pm 106$ & $1512^{\mathrm{a}} \pm 85$ & $1565^{\mathrm{b}} \pm 92$ \\
$6 \times 3 \mathrm{~min}$ at $85 \%$ & $1289^{\mathrm{a}} \pm 175$ & $1368^{\mathrm{a}, \mathrm{b}_{ \pm 189}}$ & $1408^{\mathrm{b}} \pm 174$ \\
\hline
\end{tabular}

Data are given as mean and standard deviation. Significant differences $(P<0.05)$ within one training protocol, are indicated in the horizontal direction by the superscripts $a, b, c$, meaning no difference in case of an identical letter ( $n=24$ horses; sed $=49$ ).

The results indicate that running time until exhaustion increased during the training period in three out of four groups (Table 6.1), i.e., the group with 3 work bouts at $70 \%$ of the peak heart rate did not show an improvement. It was found that in the other three groups the program with 3 exercise bouts of $3 \mathrm{~min}$ at $85 \%$ of the peak heart rate was the only one reaching the level of significance as early as day 54 . No further increase was seen between day 54 and day 106. Between day 0 and day 106 the increase of the running time until exhaustion ranged from 5-7\% of the running time on day 0. Despite a significant improvement in the groups with $3 \times 3 \mathrm{~min}$ at $85 \%$, $6 \times 3 \mathrm{~min}$ at $70 \%$ and $6 \times 3 \mathrm{~min}$ at $85 \%$, no differences between treatments were seen at the corresponding test days.

Regarding the different regimens, an increase in vertical work delivered ranging from $6-14 \%$ on day 54 was found, with only the change in the group with 3 exercise bouts of $3 \mathrm{~min}$ at $85 \%$ reaching the level of significance (14\% increase). Compared with the level on day 0 , the increase on day 106 ranged from 9-11\% (Table 6.2). This 
increase was significant in all four groups. No significant differences were present between the different treatments at corresponding test days.

The peak heart rate of the individual horses, measured during the incremental exercise test, did not change during the training period (data not shown). No change was found in the relationship between heart rate and plasma lactate as a function of power output during the experimental period in the different exercise protocols (Fig. 6.1 and 6.2). So, despite an increase in performance capacity during the experimental period, neither heart rate nor plasma lactate showed a change at the same workload in any of the training groups.

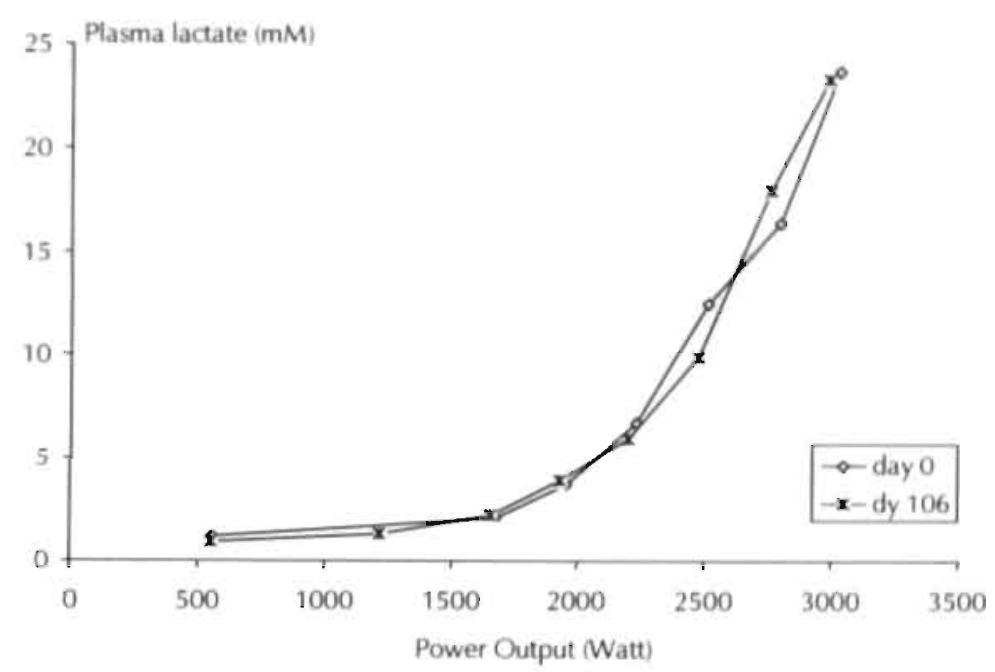

Fig, 6.1 Average plasma lactate concentration during the incremental exercise test at day 0 and day 106 of the experimental period. For the sake of clarity only mean values are given $(n=24)$

Plasma volume was found to be enlarged in all groups at the end of the experimental period (Table 6.3). Only the group with 3 repetitions at an intensity of $70 \%$ of the peak heart rate showed no increase at day. 54 . The other groups showed a significant increase in the first half of the experimental period. Thereafter no further increase was seen in these groups. 


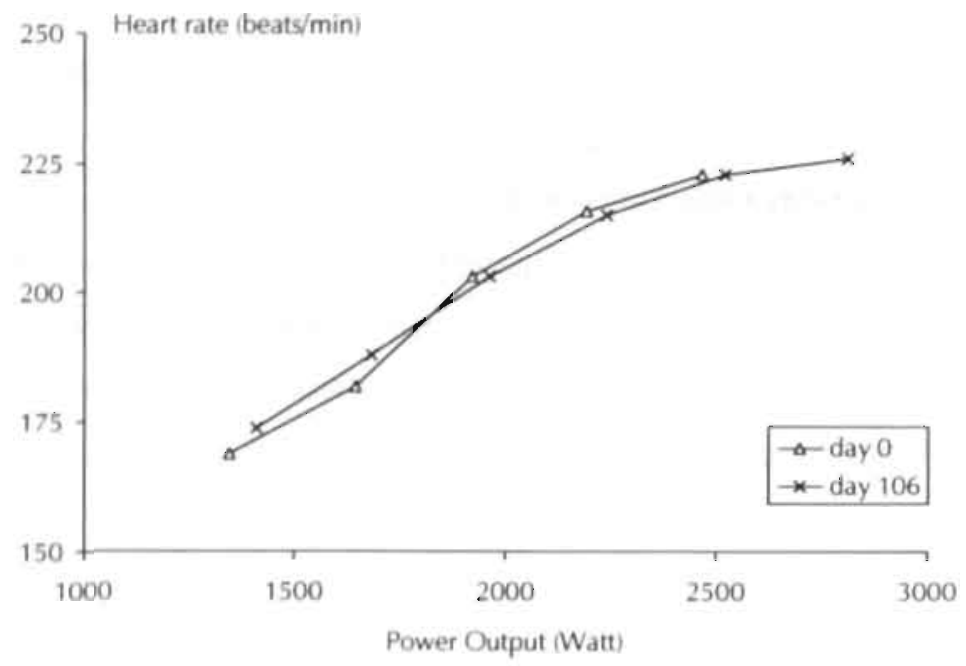

Fig. $6.2 \mathrm{M}$ oun hoart rato thears/minj during the incremental exercise test at day 0 and day 106 of the experimental period. For the sake of clarity only the mean values are presented $(n=24)$

Red blood cell volume increased significantly in all groups during the experimental period (Table 6.4). At the start of the experiment (day 0), an unexplained difference was seen between the groups, with an intensity of $70 \%$ and $85 \%$ of the peak heart rate. Still, a significant effect of exercise intensity was found with a larger change in the groups with an exercise intensity of $85 \%$ of the peak heart rate $(P<0.001)$. These groups showed a significant increase between day 0 and day 54 , and again between day 54 and day 106, while the groups with an exercise intensity of $70 \%$ of the peak heart rate showed a significant increase only over the total experimental period. 
Table 6.3 Plasma volume (I) measured immediately after the incremental exercise test of the different training protocols.

\begin{tabular}{l|ccc}
\hline & \multicolumn{3}{|c}{ Day of experimental period } \\
\hline training protocol & 0 & 54 & 106 \\
\hline $3 \times 3 \mathrm{~min}$ at $70 \%$ & $23.3^{\mathrm{a}} \pm 3.0$ & $24.7^{\mathrm{a}} \pm 5.3$ & $25.0^{\mathrm{b}} \pm 3.7$ \\
$3 \times 3 \mathrm{~min}$ at $85 \%$ & $22.0^{\mathrm{a}} \pm 3.1$ & $24.8^{\mathrm{b}} \pm 3.6$ & $26.0^{\mathrm{b}} \pm 1.5$ \\
$6 \times 3 \mathrm{~min}$ at $70 \%$ & $22.0^{\mathrm{a}} \pm 2.9$ & $24.6^{\mathrm{b}} \pm 4.5$ & $24.7^{\mathrm{b}} \pm 3.8$ \\
$6 \times 3 \mathrm{~min}$ at $85 \%$ & $20.5^{\mathrm{a}} \pm 2.7$ & $25.2^{\mathrm{b}} \pm 2.6$ & $25.0^{\mathrm{b}} \pm 2.8$ \\
\hline
\end{tabular}

Data are given as mean and standard deviation. Significant differences $(P<0.05)$ within one training protocol, are indicated in the horizontal direction by the superscripts ${ }^{a}, b, c$, meaning no difference in case of an identical letter $(n=24$ horses; sed $=0.7)$.

Table 6.4 Red blood cell volume $(\mathrm{ml} / \mathrm{kg})$ after the exercise test of the different training protocols.

\begin{tabular}{|c|c|c|c|}
\hline & \multicolumn{3}{|c|}{ Day of experimental period } \\
\hline training protocol & 0 & 54 & 106 \\
\hline $3 \times 3 \mathrm{~min}$ at $70 \%$ & $77.5^{\mathrm{a}} \pm 15.2$ & $74.4^{\mathrm{a}} \pm 19.3$ & $83.7^{b} \pm 25.8$ \\
\hline $3 \times 3$ min at $85 \%$ & $60.5^{a} y \pm 14.0$ & $75.5^{b} \times \pm 8.4$ & $87.4^{\mathrm{C}} \mathrm{xy} \pm 12.8$ \\
\hline $6 \times 3 . \min$ at $70 \%$ & $81.9^{\mathrm{a}} \times 19.7$ & $83.4^{a} \pm 25.2$ & $89.9^{\mathrm{b}} \pm 25.3$ \\
\hline $6 \times 3 \mathrm{~min}$ at $85 \%$ & $63.2^{\mathrm{a}} \mathrm{y} \pm 15.2$ & $73.7^{b} \pm 10.2$ & $86.2^{C}{ }_{x y} \pm 12.5$ \\
\hline
\end{tabular}

Data are given as mean and standard deviation. Significant differences $(P<0.05)$ within one training protocol, are indicated in the horizontal direction by the superscripts $a, b, c$, and in the vertical direction by the subscripts $x_{\imath} y, z$ meaning no difference in case of an identical letter $(n=24$ horses; sed $=2.5$ ). 


\subsubsection{Biochemical parameters in muscle biopsies}

The tissue content of some biochemical variables measured in biopsies of gluteus medius muscle showed a change during the training period, whereas no differences were seen between the different training protocols. Because of that the data of the four training groups were combined and presented for all horses in one table as values before the incremental exercise tests (Table 6.5). The values for the biopsies taken before the incremental exercise tests show an increase in resting muscle glycogen content during the experimental period. A lower resting level of ATP was observed on day 54 and day 106 compared to day 0 . The resting AMP content in muscle tissue was found to be increased on day 106 compared with day 0 and day 54 (Table 6.5). The content of the other compounds measured did not change.

Immediately after the maximal exercise test profound changes were found in the tissue content of biochemical variables. The differences in tissue levels of biochemical parameters measured in biopsies taken before and after the incremental exercise tests were also pooled, because no significant differences in the exerciseinduced changes among the four training protocols could be found (Table 6.6).

During the exercise test glycogen content decreased by approximately $30-40 \%$ (Table 6.6). Lactate levels were found to be increased from 10-12 to $75-100 \mu \mathrm{mol} / \mathrm{g}$ dry weight. Phosphocreatine levels dropped by approximately $50 \%$, while the content of ATP fell by $20-30 \%$ or $6-7 \mu \mathrm{mol} / \mathrm{g}$ dry weight. The amount of IMP increased from $0.1-0.2 \mu \mathrm{mol} / \mathrm{g}$ dry weight to values in the range of $2.0-3.0 \mu \mathrm{mol} / \mathrm{g}$ dry weight. No statistical differences with respect to the magnitude of the changes could be observed between the different test days. 
Table 6.5 Resting muscle content of Glycogen, Lactate, PCr, AIP, ADP, AMP and IMP before the incremental exercise test at different days.

\begin{tabular}{l|cccc}
\hline \multicolumn{4}{c}{ Day of experimental period } & sed \\
\hline \multicolumn{1}{c}{0} & 54 & 106 & 36 \\
\hline Glycogen & $577^{\mathrm{a}} \pm 116$ & $630^{\mathrm{ab}} \pm 119$ & $680^{\mathrm{b}} \pm 96$ & 1.9 \\
Lactate & $12.0 \pm 5.0$ & $12.1 \pm 9.3$ & $10.8 \pm 5.0$ & 4.6 \\
ATP & $80.2 \pm 1.4 .7$ & $72.2 \pm 13.6$ & $80.3 \pm 16.7$ & 0.8 \\
ADP & $28.4^{\mathrm{a}} \pm 2.2$ & $25.5^{\mathrm{b}} \pm 3.5$ & $26.7^{\mathrm{b}} \pm 2.8$ & 0.1 \\
AMP & $2.6 \pm 0.2$ & $2.3 \pm 0.4$ & $2.5 \pm 0.2$ & 0.03 \\
IMP & $0.11^{\mathrm{a}} \pm 0.06$ & $0.11^{\mathrm{a}} \pm 0.06$ & $0.21^{\mathrm{b}} \pm 0.10$ & 0.04 \\
\hline
\end{tabular}

Data are given as mean and standard deviation in $\mu$ mol/g dry weight; $n=24$ horses. $P C r$, phosphocreatine. Data of all groups were combined since no differences between groups were present. 
Table 6.6 Difference in muscle content of Glycogen, Lactate, PCr, ATP, ADP, AMP and IMP before and immediately after incremental exercise test at different days.

\begin{tabular}{l|ccc|c}
\hline \multicolumn{5}{c}{ Day of experimental period } \\
\cline { 1 - 3 } \multicolumn{1}{c}{0} & 54 & 106 & sed \\
\hline Glycogen & $253 \pm 116$ & $229 \pm 154$ & $259 \pm 140$ & 46 \\
Lactate & $-81.5 \pm 28.0$ & $-74.6 \pm 28.8$ & $-64.4 \pm 28.8$ & 8.7 \\
PCr & $51.3 \pm 23.3$ & $41.2 \pm 15.6$ & $42.6 \pm 15.2$ & 6.0 \\
ATP & $6.3 \pm 4.0$ & $7.0 \pm 5.1$ & $6.0 \pm 4.6$ & 1.6 \\
ADP & $-0.04 \pm 0.37$ & $-0.13 \pm 0.47$ & $0.07 \pm 0.39$ & 0.11 \\
AMP & $0.00 \pm 0.06$ & $-0.03 \pm 0.09$ & $-0.01 \pm 0.08$ & 0.03 \\
IMP & $-2.7 \pm 2.1$ & $-2.7 \pm 2.4$ & $-2.0 \pm 2.2$ & 0.7 \\
\hline
\end{tabular}

Data are given as mean and standard deviation in $\mu$ mol/g dry weight; $n=24$ horses. $P C r$, phosphocreatine. Data of all groups were combined since no differences between groups were present.

\subsection{DISCUSSION}

The main purpose of the present investigation was to study in horșes the effect of aerobic training protocols, varying in intensity and number of repetitions, on performance capacity. In addition, it was attempted to relate possible improvement of performance capacity to changes in other relevant physiological variables.

Heart rate recordings showed that the intensity of the work bouts in the different groups corresponded well with the target intensities of $70 \%$ and $85 \%$ of the peak heart rate. Plasma lactate levels proved to remain below $4 \mathrm{mM}$. This indicates 
that the maximal (averaged) intensity of $87 \%$ of the peak heart rate still resulted in mainly aerobic exercise, supporting the findings of Lindholm et al. $(14,15)$ and Asheim et al. (1).

The performance capacity, measured as the total amount of vertical work during the incremental exercise test, increased in all groups over a period of 105 days. This suggests that 3 bouts at $70 \%$ of the peak heart rate already provides a sufficient stimulus to increase aerobic working capacity in horses. These results are in line with those of Knight et al. (13), who studied the effect of exercise intensity on $\mathrm{VO}_{2 \max }$. These investigators compared continuous exercise at intensities of $40 \%$ and $80 \%$ of the maximal oxygen uptake and observed a $10 \%$ increase of the $\mathrm{VO}_{\min }$ after two weeks of training in both groups without a difference between the two training protocols. Interestingly, in the present study the fastest effect of training on performance capacity was observed in horses undergoing a training protocol that consisted of 3 times 3-min exercise at $85 \%$ of $\mathrm{VO}_{2 \max }$. Both running time until exhaustion and amount of vertical work showed already after 54 days of training a statistically significant improvement (Table 6.1 and 6.2).

Peak heart rates did not alter during the experimental period which is in agreement with experimental findings of others $(7,8)$. Therefore, it appears to be justified to use the relative heart rate (expressed as a percentage of the peak heart rate) as a parameter for the exercise intensity throughout the training period.

No change in the relation between heart rate and power output and plasma lactate and power output occurred during the experimental period. This is in agreement with the findings of Knight et al. (13), who failed to find a change in $V_{200}$ comparing two programs with aerobic training, and also in line with findings of other investigators (7, 18, 26). In contrast, Foreman et al. (8) and Thornton et al. (32) reported a decrease in heart rates at submaximal exercise after a period of training. This discrepancy may be caused by differences in training status at the beginning of the training period. The more untrained the animals are at the onset of the training, the 
larger the shifts in the relationship between power output and plasma lactate levels will be (2).

To obtain insight into the possible mechanism underlying changes in performance capacity, a number of relevant physiological variables was determined. Plasma volume and red blood cell volume are determining factors for training and aerobic working capacity (22). Plasma volume increased between $7 \%$ and $22 \%$ during the training period in the different exercise groups (Table 6.3). The changes in plasma volume were consistent with the findings of Persson (22), Knight et al. (13), McKeever et al. (17) and Roneus et al. (25). An increase of red blood cell volume between $8 \%$ and $44 \%$ was seen in the different groups (Table 6.4), which is in accordance with the finding of Persson et al. (23). Both parameters showed the fastest rise in the group with 3 repetitions at an exercise intensity of $85 \%$ of the peak heart rate, suggesting that exercise at relatively high intensities at the start of a training program is instrumental in enlarging red blood cell volume and consequently in improving oxygen transport capacity.

Irrespective of exercise protocol, an increase in resting muscle glycogen content was seen during the training protocol. This corroborates the findings in human volunteers subjected to aerobic exercise (for references see 28) and the findings of Lindholm et al. (14, 15), Nimmo et al. (19), Guy et al. (11) and foreman et al. (8) in horses. Increased tissue levels of glycogen is considered to be an important metabolic adaptation in muscles, which will improve the endurance time of high intensity exercise because of an enlarged pool of available carbohydrate substrates for glycolytic energy conversion.

The resting tissue level of ATP showed a small but significant decline during the training period. No explanation can be offered for this rather unexpected phenomenon. At first sight, a decline in this important high-energy phosphate may be disadvantageous for proper mechanical function of the skeletal muscle cell. However, 
it should be taken into account that in addition to the absolute level of ATP, the turnover of the cellular ATP is of great importance for the conversion of chemical into mechanical energy in the skeletal muscle cell. The present experimental set-up, however, does not allow for conclusions regarding the effect of training on ATP turnover rates.

Interestingly, resting skeletal muscle content of AMP showed a significant increase at the end of the training protocol. Although this change was found to be small in absolute terms, it cannot be excluded that an increase in the cellular content of this important regulating agent affects metabolic energy conversion in the (resting) muscle. As AMP influences the rate of both glycogenolysis and the glycolytic pathway, the training protocols applied in the present study may exert a positive effect on glucose metabolism in equine skeletal muscles (5).

In general, the other biochemical parameters measured in the middle gluteal muscle did not show a consistent change during the course of the training period. These findings strongly suggest that the level of the high-energy phosphates and related compounds is not a major limiting factor for obtaining a higher performance capacity during aerobic exercise programs at different intensities and repetitions of exercise bouts, as used in this experiment.

The magnitude of the decline in tissue glycogen and phosphocreatine and ATP, and the increase of tissue lactate and IMP, and the basically unchanged levels of ADP and AMP during the incremental exercise test did not differ between either the various. exercise protocols or during the course of the training period. This finding shows that energy metabolism very much adapts to the significantly increased work (i.e., amount of vertical work and running time until exhaustion) performed during the incremental exercise test.

The increase in tissue IMP associated with decreased ATP levels indicates that the energy production is challenged with enhanced myokinase activity to generate anaerobically ATP from ADP, which results in the formation of AMP and subsequently 
IMP. These findings are in line with the observations of Sahlin et al. (27) and Sewell et al. (29). The extent of glycogen consumption during the incremental exercise tests was of the same magnitude as described by Lindholm et al. (14), Gottlieb (10), Nimmo et al. (20) and Harris et al. (12). The change in lactate content in muscle tissue during the exercise tests corroborates the findings of Valberg et al (33), Snow et al. (31) and Sewell et al. (29).

In conclusion, the data of the present study demonstrate that in horses an aerobic training protocol, consisting of 3 or 6 times 3 -min work bouts at intensities of either $70 \%$ or $85 \%$ of the peak heart rate, results in an improved performance capacity of about $10 \%$ when amount of vertical work delivered during a treadmill test was considered. This improvement was not influenced by the intensity and/or number of the work bouts over a period of 105 days. A significant contribution to practical training is that the effect on performance capacity is not linearly related to intensity and volume of training. Because of the effect of exercise intensity on the increase in red blood cell volume, with a faster adaptation at the higher exercise intensity, it is advised to use an intensity of $85 \%$ of the maximal heart rate. The number of repetitions should be kept as low as possible to prevent injuries. Three repetitions as used in this study proved to be sufficient for the increase in oxygen transport capacity.

\subsection{REFERENCES}

1. Asheim A., O. Knudsen, A. Lindholm, O. Rülcker, B. Saltin. Heart rates and blood lactate concentrations of standardbred horses during training and racing. I Am Vet Med Assoc 157: 304-312, 1970.

2. Astrand P.O., K. Rodahl. Textbook of Work Physiology. Physiological bases of exercise. McGraw-Hill Book Company, New York, 1986.

3. Bergstrom J.. Percutaneous needle biopsy of skeletal muscle in physiological and clinical research. Scand J Clin Invest 35: 609-616, 1975. 
4. Blomqvist C.G., B. Saltin. Cardiovascular adaptations to physical training. Ann Rev Physiol 45:169-189, 1983.

5. Devlin, T.M.. Textbook of Biochemistry. Wiley-Liss, New York, 1992.

6. Essen-Gustavsson B., D. McMiken, K. Karlstrom, A. Lindholm, S.G.B. Persson, I. Thomton. Muscular adaptation of horses during intensive training and detraining. Eq Vet |, 21: 27-33, 1989.

7. Evans D.L., R.J. Rose. Cardiovascular and respiratory reponses to submaximal exercise training in the thoroughbred horse. Pflügers Arch 411: 316-321, 1988.

8. Foreman J.H., W.M. Bayly, B.D. Grant, P.D. Gollnick. Standardized Exercise Test and Daily Heart Rate Responses of Thoroughbreds Undergoing Conventional Race Training and Detraining. Am J Vet Res 51: 914-920, 1990.

9. Genstat 5 Committee (R.W. Payne, chairman, P.W. Lane, secretary). Design and analysis of experiments. In: Genstat 5 Release 3 Reference Manual, Clarendon Press, Oxford, 1993, pp. 461-538.

10. Gottlieb M.. Muscle glycogen depletion patterns during draught work in Standardbred horses. Eq Vet J 21: 110-115, 1989

11. Guy P.S., D.H. Snow. The effect of training and detraining on muscle composition in the horse. J Physiol 269: 33-51, 1977.

12. Harris R.C., D.J. Marlin, D.H. Snow. Metabolic response to maximal exercise of 800 and 2000 $m$ in the thoroughbred horse. J Appl Physiol 63: 12-19, 1987.

13. Knight P.K., A.K. Sinha, R.J. Rose. Effects of training intensity on maximum oxygen uptake. in: Persson S.G.B., A. Lindholm. L.B. Jeffcott (eds): Equine Exercise Physiology 3, Almquist \& Wiksell Tryckeri, Uppsala, 1991, pp. 77-82.

14. Lindholm A., K. Piehl. Fibre composition, enzyme activity and concentrations of metabolites and electrolytes in muscles of standardbred horses. Acta Vet Scand 15: 287-309, 1974.

15. Lindholm A., H. Bjerneld, B. Saltin. Glycogen depletion pattern in muscle fibre of trotting horses. Acta Physiol Scand 90: 475-484, 1974.

16. McArdle W.D., F.I. Katch, V.L. Katch. Training for anaerobic and aerobic power. In: Exercise Physiology. Energy, nutrition and human performance, Lea \& Febiger, Philadelphia, 1986, pp. 347-370. 
17. McKeever K.H., W.A. Schurg, S.H. Jarrett, V.A. Convertino. Exercise training-induced hypervolemia in the horse. Med Sci Sports Exerc 19: 21-27, 1987.

18. Milne D.W., A.A. Gabel, W.W. Muir, R.T. Skarda. Effect of training on heart rate, cardiac output, and lactic acid in standardbred horses using a standardized exercise test. J Eq Med Surg 1: 131-135, 1977.

19. Nimmo M.A., D.H. Snow, C.D. Munro. Effects of nandrolone phenylpropionate in the horse: (3) Skeletal muscle composition in the exercising animal. Eq Vet ) 14: 229-233, 1982.

20. Nimmo M.A., D.H. Snow. Changes in muscle glycogen, lactate and pyruvate following maximal exercise in the thoroughbred horse. In: Snow D.H., S.G.B. Persson, R.J. Rose (eds): Equine Exercise Physiology, Burlington Press, Cambridge, 1983, pp. 237-244.

21. Passoneau J.V... Lactate. Fluorometric method. In: Bergmeyer H.U. (ed): Methods of Enzymatic Analysis, New York Academic Press, New York, 1974, pp. 1464-1472, .

22. Persson S.G.B.. On blood volume and working capacity in horses. Acta Vet Scand Suppl 19: 12-180, 1967 .

23. Persson S.G.B., L.E. Ullberg. Blood volume in relation to exercise tolerance in trotters. I S Afr Vet Assoc 45: 293-299, 1974.

24. Physick-Sheard P.W.. Cardiovascular response to exercise and training in the horse. Vet Clin North Am Eq Pract 1: 383-417, 1985.

25. Roneus M., B. Essen-Gustavsson, A. Lindholm, S.G.B. Persson. A Field Study of Circulatory Response and Muscle Characteristics in Young Thoroughbreds. In Gillespie J.R., N.E. Robinson (eds): Equine Exercise Physiology 2 Edition, Edwards Brothers, Ann Arbor, USA, 1987, pp. 376-383.

26. Rose R.J. J.R. Allen, D.R. Hodgson, J.H. Stewart, W. Chan. Responses to submaximal treadmill exercise and training in the horse: Changes in haematology, arterial blood gas and acid base measurements. Vet Rec 113: 612-618, 1983.

27. Sahlin K., S. Broberg, J.M. Ren. Formation of inosine monophosfate (IMP) in human skeletal muscle during incremental dynamic exercise. Acta Physiol Scand 136: 193-198, 1989.

28. Saltin, B., P.D. Gollnick. Skeletal muscle adaptibility: Significance for metabolism and performance In: L.D. Peachy (ed.): Handbook of Physiology, Section 10. Skeletal muscle. Williams and Wilkins, Baltimore, 1983, pp. 555-631. 
29. Sewell D.A., R.C. Harris, J. Hanak, P. Jahn. Muscle Adenine Nucleotide Degradation in the Thoroughbred Horse as a Consequence of Racing. Comp Biochem Physiol 101: 375-381. 1992.

30. Snedecor G.W., W.G. Cochran. Statistical Methods. The lowa State University Press, Ames, lowa, USA, 1989.

31. Snow D.H., R.C. Harris, S.P. Gash. Metabolic response of equine muscle to intermittent maximal exercise. J Appl Physiol 58: 1689-1697, 1985.

32. Thornton J., B. Essen-Gustavsson, A. Lindholm, D. McMiken, S.G.B. Persson. Effects of Training and Detraining on Oxygen Uptake, Cardiac Output, Blood Gas Tensions, $\mathrm{pH}$ and Lactate Concentrations during and after Exercise in the Horse. In: Snow D.H., S.G.B. Persson, R.J. Rose (eds): Equine Exercise Physiology, Burlington Press, Cambridge, 1983, pp. 470-486.

33. Valberg S., B. Essen-Gustavsson. Metabolic Response to Racing Determined in Pools of Type I, IIA and IIB Fibers. In: Gillespie, N.E. Robinson (eds): Equine Exercise Physiology 2 Edition, Edwards Brothers, Ann Arbor, USA, 1987, pp. 290-301.

34. Valberg S., B. Essen-Gustavsson, A. Lindholm, S.G.B. Persson. Blood chemistry and skeletal muscle metabolic responses during and after different speeds and durations of trotting. Eq Vet J 21: 91-95, 1989.

35. Van der Vusse G.J., W.A. Coumans, F.H. Van der Veen, A. Drake. ATP, creatine phosphate and glycogen content in human myocardial biopsies: markers for the efficacy of cardioprotection during aorta coronary bypass surgery. Vasc Surg 18: 127-134, 1984.

36. Van der Vusse G.J., G.M.E. Janssen, W.A. Coumans, H. Kuipers, R.J.M.M. Does, F. Ten Hoor. Effect of training and 15-, 25- and $42 \mathrm{~km}$ contests on the skeletal muscle content of adenine an guanine nucleocides, creatine phosphate and glycogen. Int J Sports Med 10: S146-S152, 1989.

37. Wijnants J., H. Van Belle. Single-run high performance liquid chromatography of nucleotides, nucleosides and major purine bases and its application to different tissue extracts. Anal Biochem 144: 258-266, 1985. 
Chapter 6 . 


\section{EFFECTS OF 1-MIN ANAEROBIC EXERCISE BOUTS, ADDED TO AEROBIC INTERVAL TRAINING OF DIFFERENT INTENSITY AND DURATION, ON THE PERFORMANCE CAPACITY OF HORSES}

This chapter will be published as:

G. Bruin, H. Kuipers, G. André, and G.J. van der Vusse

Effects of 1-min anaerobic exercise bouts, added to aerobic interval training of different intensity and duration, on the performance capacity of horses Int J Sports Med (Accepted for publication) 


\subsection{ABSTRACT}

In a randomised block design, 12 Standardbred trotter stallions were used to investigate the effect of aerobic exercise at two different intensity levels, combined with six 1-minute anaerobic exercise bouts, on the performance capacity of horses. Performance capacity was expressed as the regression of heart rate versus power output, plasma lactate versus power output, total running time until exhaustion, and the total amount of vertical work. In addition, a number of biochemical variables was measured in biopsy samples from the middle gluteal muscle. The horses were trained three times a week during a period of 106 days on a treadmill with a $6.25 \%$ incline. The training protocols consisted of six 3-minute intervals at $70 \%$ of the peak heart rate or three 3minute intervals at $85 \%$ of the peak heart rate combined with 6 intensive exercise bouts of 60 seconds at a heart rate $>90 \%$ of the peak heart rate. Before the experiment and at day 40, 71 and 106, the horses performed a stepwise increasing exercise test until exhaustion on the treadmill.

None of the exercise protocols resulted in a significant improvement of the performance capacity. In addition no changes in other indices of performance and of biochemical markers in blood plasma were found between the four training protocols. Overall, in muscle biopsies the glycogen content showed an increase during the training period, while during the incremental exercise tests the amount of glycogen and ATP decreased and the amount of IMP increased. The increase in lactate and IMP during the exercise test at day 106 was found to be less pronounced in the groups with anaerobic components in their training protocol.

In conclusion, the results suggest that training protocols consisting of aerobic intervals combined with six 1-minute sprints resulting in plasma lactate levels of 7-8 $\mathrm{mM}$, are not sufficient to increase performance capacity. This might indicate that exercise resulting in plasma lactate levels below $10 \mathrm{mM}$ is ineffective for improving performance capacity. Most likely, exercise bouts resulting in plasma lactate levels above $10 \mathrm{mM}$ are necessary to realise the maximal performance capacity of horses. 


\subsection{INTRODUCTION}

Races with Standardbred trotters and Thoroughbreds have a duration between $\sim 1$ and $\sim 10$ minutes and challenge aerobic as well as anaerobic energy conversion. Training exercise of these horses should therefore include both anaerobic and aerobic exercise $(10,11,12)$. To improve the aerobic capacity in humans an exercise intensity between $70 \%$ and $85 \%$ of the maximal heart rate is necessary (13). Anaerobic training in horses as well as in humans requires intensities beyond $85 \%$ of the peak heart rate $(11,13$, see also chapter 6$)$. The disadvantage of anaerobic exercise is that it rapidly induces fatigue (1). In a previous study (chapter 6) it was shown that intermittent aerobic exercise bouts do increase performance capacity. However, it is unknown whether adding anaerobic bouts exercise yields a higher increase in performance capacity, In horse training limited research has been done to study the effect of different training regimens including both aerobic and anaerobic components. Usually, based on tradition and intuition a mixed training program is applied consisting of aerobic endurance type of training combined with high intensity interval training $(4,5$, $8,14,15,16,17)$.

Therefore, in the present study the physiological response was studied of a training program as used in horse practice consisting of aerobic exercise of different intensity, to which none or 6 intensive, anaerobic exercise bouts were added. In order to obtain insight into the physiological basis of possible changes in performance capacity a number of physiological and biochemical variables was measured, such as plasma volume, red blood cell volume, and the skeletal muscle content of glycogen, lactate, energy-rich phosphates and related compounds.

\subsection{MATERIAL AND METHODS.}

\subsubsection{Horses and training}

Twelve two-year-old Standardbred trotter stallions were used. The horses participated in a previous study. To reverse training effects, two months of low 
intensity endurance exercise at an intensity lower than $60 \%$ of the peak heart rate was given for 5 days a week. In a randomised block design the horses were assigned to one of the four different training protocols (see below). Heart rate was used to determine the exercise intensity. The exercise intensity was based on fixed percentages of the peak heart rate. The peak heart rate was the maximal heart rate ever measured in an individual horse during an exercise test until exhaustion.

The four different training protocols, with 3 horses each, consisted of:

group 1: $\quad 6$ bouts of 3 min at $70 \%$ interspersed with 4 min at $60 \%$ of the peak heart rate (protocol 6_70_0):

group 2: $\quad 6$ bouts of $3 \mathrm{~min}$ at $70 \%$ interspersed with $4 \mathrm{~min}$ at $60 \%$ of the peak heart rate combined with 6 intervals of $1 \mathrm{~min}$ with a heart rate $>90 \%$ of the peak heart rate (protocol 6_70_6);

group 3: $\quad 3$ bouts of $3 \mathrm{~min}$ at $85 \%$ interspersed with $4 \mathrm{~min}$ at $60 \%$ of the peak heart rate (protocol 3_85_0);

group 4: $\quad 3$ bouts of $3 \mathrm{~min}$ at $85 \%$ interspersed with 4 min at $60 \%$ of the peak heart rate combined with 6 intervals of 1 min with a heart rate $>90 \%$ of the peak heart rate (protocol 3_85_6).

The different training protocols are schematically represented in figures 7.1 through 7.4.

Each training session was preceded by a $6 \mathrm{~min}$ warming-up at $4.5 \mathrm{~m} / \mathrm{s}$. The four different training protocols were performed 3 times a week for a period of 106 days on a treadmill with an inclination of $6.25 \%$. The days with treadmill training were interspersed with days on which endurance running at an intensity lower than $60 \%$ of the peak heart rate was performed in a rotary exerciser. In the afternoon of the days with treadmill exercise the horses performed an exercise bout of $30 \mathrm{~min}$ at $1.5 \mathrm{~m} / \mathrm{s}$ in a rotary exerciser. In total the horses were exercised for 6 days per week with 1 day without any exercise. Once a week the speed of the treadmill during the interval bouts and endurance exercise was adjusted according to the results of training and tests in order to obtain the desired level of 70 or $85 \%$ of the peak heart rate. During the first three weeks of the experimental period the intensity of the sprints was gradually 
increased to the target level. The horses were fed according to the energy requirements and individually housed.

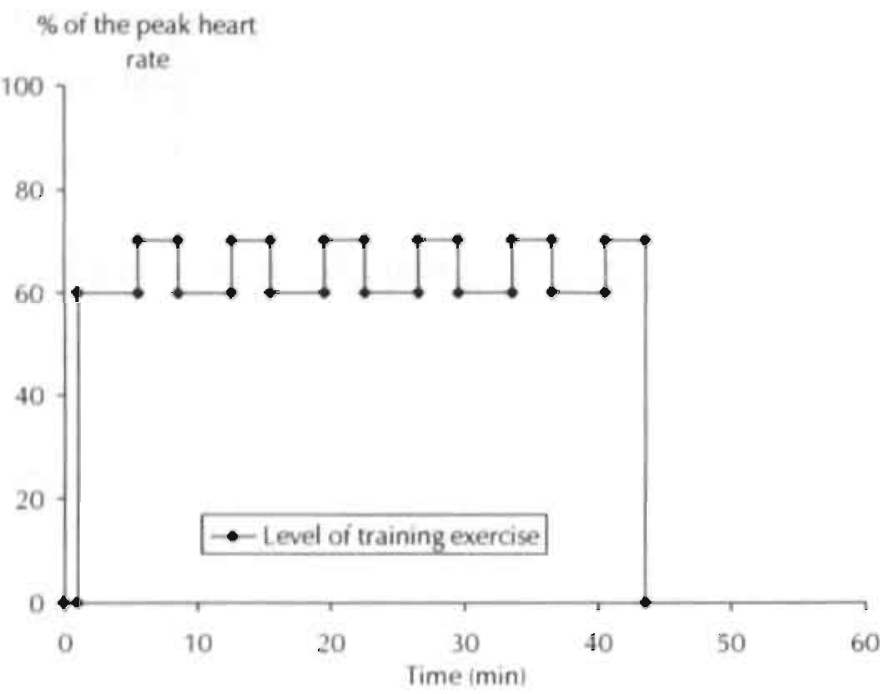

Fig. 7.1 Training protocol group 1 (6_70_0)

$\%$ of the peak heart

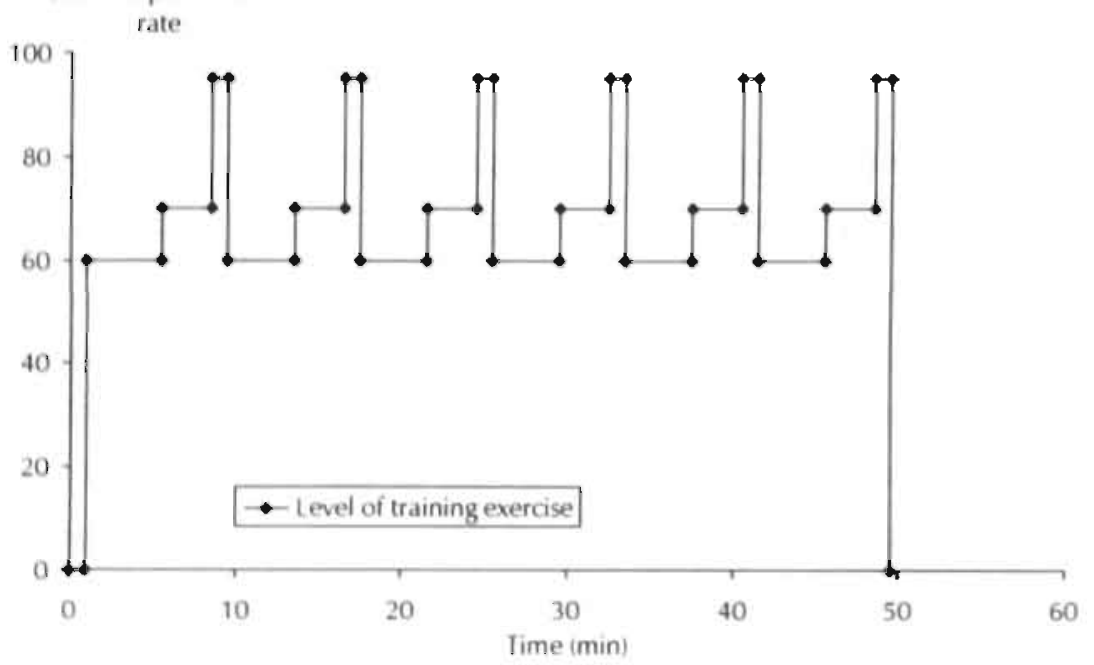

Fig. 7.2 Training protocol group 2 (6_70_6) 


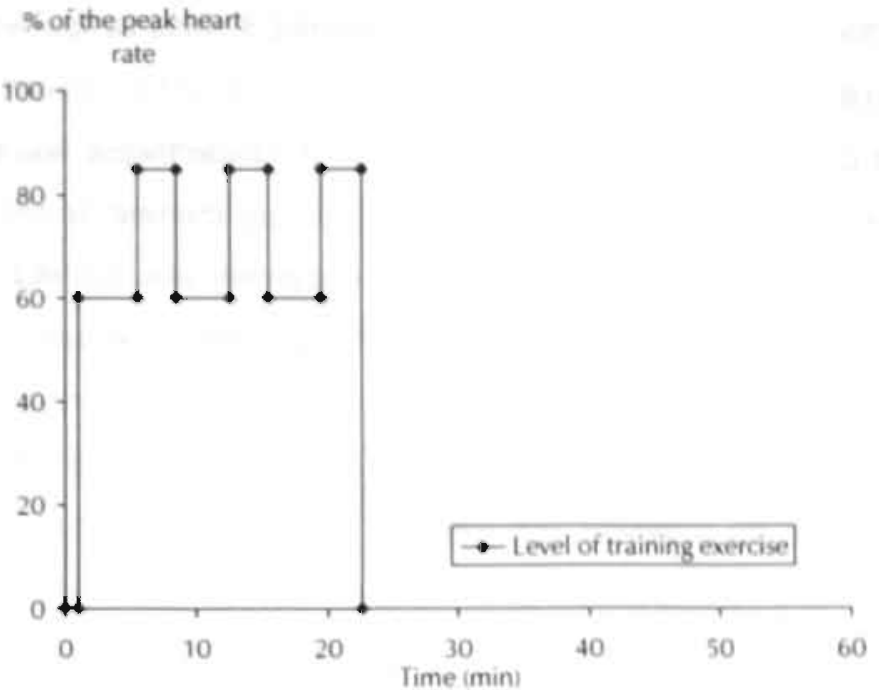

Fig. 7.3 Training protocol group 3 (3_85.0)

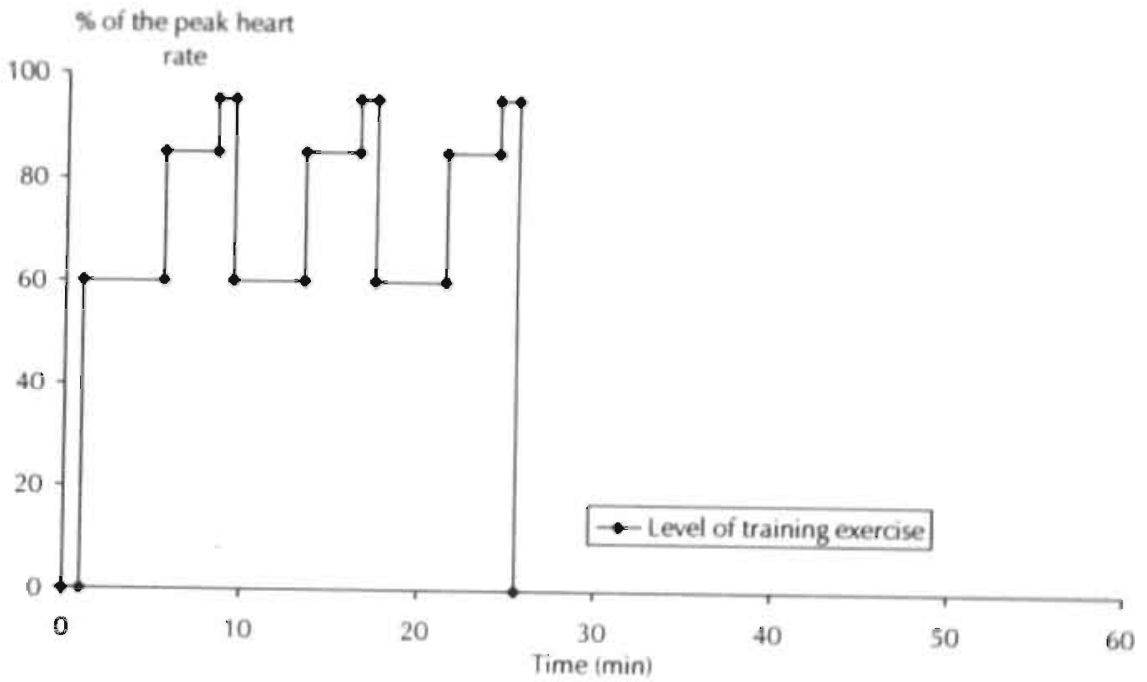

Fig. 7.4 Training protocol group 4 (3_85_6)

\subsubsection{Working procedure}

Every day at 8 a.m. the weight of the horses was measured. On training days the horses were prepared by attaching the safety belt and the belt for the heart rate 
monitor. Two and five minutes after stopping the training a blood sample was taken to determine the plasma concentration of lactate.

On test days the horse was catheterised and a muscle biopsy was taken from the gluteus medius muscle $(2,4,10)$. The safety belt and the belt for the heart rate monitor were attached. On the treadmill the ecg-equipment was also attached to the belt of the heart rate monitor. Blood samples were taken prior to exercise, during the last $15 \mathrm{~s}$ of each step, and 2 and $5 \mathrm{~min}$ after finishing the test. Immediately after the maximal exercise test a muscle biopsy was taken and the temperature was measured. After that, the horse got a shower, was weighed and put into the housing box. The tests were performed between $7 \mathrm{a} . \mathrm{m}$. and noon.

\section{$\underline{7.3 .3 \text { Incremental exercise test and data collection }}$}

Four incremental exercise tests were done on the treadmill (day 0, day 40, day 71 and day 106). The incremental exercise tests were performed after 1 day of rest. After a $6 . \mathrm{min}$ warming-up at $4.5 \mathrm{~m} / \mathrm{s}$, the incremental exercise tests consisted of a 2 min step at a treadmill speed of $2 \mathrm{~m} / \mathrm{s}$. Thereafter the speed was increased to $5 \mathrm{~m} / \mathrm{s}$ and every $2 \mathrm{~min}$ further increased with $1 \mathrm{~m} / \mathrm{s}$ until exhaustion. Exhaustion was considered to be reached if despite encouragement, the horse was not able to maintain the running speed.

During training and during the incremental exercise test the heart rate was continuously recorded every 5 s by means of the "Sport tester PE3000" (Polar Electro) and during the incremental exercise tests also by means of an electrocardiogram (ecg) (Schwarzer CS6000). During the incremental exercise test time to exhaustion was measured. Since the treadmill had a $6.25 \%$ inclination, the power output and the total amount of vertical work delivered until exhaustion could be calculated.

The power output (Watt) is calculated according to the formula:

$$
\text { power }=9.81 \times \text { body weight } \times \text { speed } \times \sin \alpha
$$

In this formula body-weight is expressed in $\mathrm{kg}$, speed in $\mathrm{m} / \mathrm{s}$, and $\alpha$ is the angle (degrees) of inclination of the treadmill.

The amount of vertical work (kJ) per stage is calculated according to the formula: 


\section{work $=9.81 \times$ body-weight $\times$ speed $\times$ duration $\times \sin \alpha$}

in which duration is the running time (s) at each treadmill speed. The amount of work at each speed is summated, resulting in the total amount of work. Only the vertical component was included.

The relationship between heart rate and power output is described by the linear relation $(H R=$ heart rate in beats $/$ min; power $=$ power output in Watt; $\beta$ $=$ intercept;

$\beta_{1}=$ regression coefficient):

$$
H R=\beta_{0}+\beta_{1} \times \text { power }
$$

The relationship between plasma lactate concentration $(\mathrm{mM})$ and power output (Watt) is described by the exponential curve:

$$
\text { lactate }=A+B \times R^{\text {power }}
$$

in which lactate is the plasma lactate concentration $(\mathrm{mM}), \mathrm{A}, \mathrm{B}$ and $\mathrm{R}$ are regression parameters, and power is the power output (Watt).

Changes in the performance capacity were measured as changes in the regression of heart rate (beats/min) versus power output (Watt), plasma lactate (mM) versus power output (Watt), the total running time until exhaustion (s), and the total amount of vertical work until exhaustion ( $k !)$.

\section{$\underline{7.3 .4}$ Measurement of plasma lactate and muscle enzymes}

The blood samples were centrifuged in heparinized Eppendorf-cups at 12,000 revolutions per min $(8800 \mathrm{~g})$. The plasma was immediately frozen at $-20^{\circ} \mathrm{C}$ and stored at $-80^{\circ} \mathrm{C}$. Plasma lactate was determined with a Lactate analyser 640 (La Roche). Muscle enzymes were determined as indicated in chapter 6.

\subsubsection{Measurement of energy-rich phosphates, glycogen and lactate}

The metabolic status of the muscular tissue was judged by determination of the amount of phosphocreatine (PCr), adenosine triphosphate (ATP), adenosine diphosphate (ADP), adenosine monophosphate (AMP), and inosine monophosphate (IMP) in biopsies obtained from the gluteus medius muscle. Also the tissue content of glycogen and lactate was determined. 
The muscle tissue of the biopsy was rapidly frozen in liquid nitrogen. Time interval between sampling of tissue and freezing was in the order of 5-10 s. The frozen tissue samples were stored at $-80^{\circ} \mathrm{C}$. Prior to biochemical analysis the samples were freeze-dried at $-30^{\circ} \mathrm{C}$ overnight.

Analysis of the tissue content of adenine nucleotides, phosphocreatine, glycogen, lactate and glucose was performed according to the techniques described in detail in chapter 6.

\subsubsection{Statistics}

The experiments were performed in a completely randomised block design. Blocks were based on performance capacity immediately before the start of the experiment. In this experiment 12 horses were used.

Analysis was performed, using the Genstat package (6), as described in detail in chapter 6. Differences were regarded significant at $P<0.05$. The heterogeneity between horses proved to be large and blocks did not reduce the experimental error.

\subsection{RESULTS}

\subsubsection{Acceptance of training protocol and training intensity}

The training was, as expected, very well accepted by the horses included in the study. All the horses could perform the planned number of treadmill sessions, while no serious health problems were observed during, the experimental period. The blood plasma level of muscle enzymes (CK, AST, LDH) and liver ALT were found to be within normal ranges and without systematic changes (data not shown).

Analysis of the heart rate recordings revealed that the horses in the groups with an exercise intensity of $70 \%$ of the maximal peak heart rate had actually trained with an intensity of 73 and $72 \%$ of the peak heart rate for no and 6 sprints, respectively. The groups with nominally $85 \%$ of the peak heart rate trained at 87 and $86 \%$ of the peak heart rate for no and 6 sprints, respectively. Heart rates at the end of the 1-min sprint exercise were actually at 94 and $96 \%$ of the peak heart rate for the groups with aerobic exercise at 70 and $85 \%$ of the peak heart rate, respectively. 
Plasma lactate levels, at the end of the interval training, reached an average value of $2.5 \pm 1.3,7.1 \pm 2.6,4.3 \pm 1.4$, and $7.4 \pm 4.1 \mathrm{mM}$ in the protocols [6_70_0], [6_70_6], [3_85_0], and [3_85_66], respectively (mean \pm sd).

\subsubsection{Incremental exercise test and plasma lactate}

Table 7.1 and 7.2 show the findings of running time until exhaustion and the amount of vertical work of the different training protocols in the incremental exercise tests on day $0,40,71$ and 106.

The results indicate that running time until exhaustion did not change in any of the four groups during the experimental period. At the onset of the experiment at day 0 no significant differences were found in total running time between the four groups. At day 40 running time until exhaustion was significantly larger in group [3_85_6] than in the two groups with an intensity of the aerobic exercise of $70 \%$ of the peak heart rate. At day 106, a significant difference was observed between group [3_85_6] and group [6_70_6], being a longer time duration in the group with the higher intensity of the aerobic exercise.

The amount of vertical work until exhaustion showed the same pattern as the running time until exhaustion, i.e. no change during the experimental period (Table 7.2). Already at the start of the experiment differences between groups of horses subjected to different training protocols could be seen, with group [3_85_0] at a higher level than group [6_70_0] (Table 7.2). This difference remained at all test days, except day 71 . On day 71 group [3_85_0] showed a level only significantly different from group [6_70_6]. No consistent patterns were found indicating a different effect of the sprint exercise if combined with a larger number of aerobic intervals at a lower exercise intensity than if combined with a lower number of aerobic intervals at a higher intensity, i.e. 6 intervals at an intensity of $70 \%$ of the peak heart rate in stead of 3 intervals at an intensity of $85 \%$ of the peak heart rate.

The peak heart rate of the individual horses did not change during the experimental period. The relationship between heart rate and power output or the relationship between plasma lactate and power output did not change, either (data not shown). 
Table 7.1 Running time until exhaustion (s) during the incremental exercise test in the different exercise protocols (meantsd).

\begin{tabular}{l|cccc}
\hline & \multicolumn{4}{|c}{ Day of the experimental period } \\
\hline protocol & 0 & 40 & 71 & 106 \\
\hline [3_85_0] & $758 \pm 23$ & $763_{x y} \pm 31$ & $750 \pm 46$ & $771_{x y} \pm 49$ \\
[3_85_6] & $770 \pm 39$ & $795_{x} \pm 43$ & $759 \pm 56$ & $795_{x} \pm 58$ \\
[6_70_0] & $759 \pm 23$ & $735_{y} \pm 47$ & $755 \pm 14$ & $763_{x y} \pm 53$ \\
[6_70_6] & $746 \pm 32$ & $745_{y} \pm 57$ & $729 \pm 44$ & $734_{y} \pm 76$ \\
\hline
\end{tabular}

Significant differences. $(P<0.05)$ are indicated in the vertical direction by the subscripts $x, y$, meaning no difference in case of an identical letter $(n=3$, in each group; standard error of difference of means $($ sed $)=21$ ). No differences could be observed between the different test days within a particular group.

Table 7.2 Amount of vertical work until exhaustion (kJ) during the incremental exercise test in the different exercise protocols (meantsd).

\begin{tabular}{c|cccc}
\hline & \multicolumn{4}{|c}{ Day of the experimental period } \\
\hline protocol & 0 & 40 & 71 & 106 \\
\hline$\left[3 \_85 \_0\right]$ & $1528_{x} \pm 4$ & $1551_{x} \pm 29$ & $1512_{x} \pm 78$ & $1593_{x} \pm 102$. \\
{$\left[3 \_85 \_6\right]$} & $1412_{x y} \pm 150$ & $1464_{x z} \pm 167$ & $1382_{x y} \pm 219$ & $1500_{x y} \pm 229$ \\
{$\left[6 \_70 \_0\right]$} & $1381_{y} \pm 123$ & $1304_{y} \pm 184$ & $1380_{x y} \pm 102$ & $1421_{y} \pm 219$ \\
{$\left[6 \_70 \_6\right]$} & $1398_{x y} \pm 99$ & $1388_{y z} \pm 217$ & $1351_{y} \pm 113$ & $1388_{y} \pm 148$ \\
\hline
\end{tabular}

Significant differences $(P<0.05)$ are indicated in the vertical direction by the subscripts $x, y, z$ meaning no difference in case of an identical letter $(n=3$ in each group; sed $=72)$. No difference could be observed between the different test days within a particular group. 


\subsubsection{Biochemical parameters in muscle biopsies.}

No differences in the resting values of the tissue content of phosphocreatine, ATP, ADP, AMP, IMP, and glycogen between day 0 and 106 could be observed in the four individual groups (data not shown).

Because of the lack of difference between the different training protocols, as far as biochemical markers are concerned (with the exception of IMP and lactate, see below), the data obtained in the four groups were combined in an attempt to disclose a general pattern of the effect of training on muscle glycogen and high energy phosphate stores. As expected resting muscle values of glycogen significantly increased during the training period. With respect to the other biochemical parameters no consistent pattern could be observed (Table 7.3).

Table 7.3 Mean muscle glycogen, energy rich phosphates, and muscle lactate before the exercise test (resting values; mean \pm sd and sed). Data obtained in the four groups subjected to different training protocols were pooled.

\begin{tabular}{|c|c|c|c|c|c|}
\hline \multicolumn{6}{|c|}{ Day of the experimental period } \\
\hline & 0 & 40 & 71 & 106 & sed \\
\hline Glycogen & $634^{\mathrm{a}} \pm 145$ & $767^{\mathrm{ab}} \pm 137$ & $734^{\mathrm{ab}} \pm 227$ & $769^{b} \pm 153$ & 66.3 \\
\hline Lactate & $13.4 \pm 74$ & $11.3 \pm 4.4$ & $14.9 \pm 5.7$ & $11.5 \pm 6.3$ & 2.05 \\
\hline IMP & $0.14 \pm 0.10$ & $0.13 \pm 0.08$ & $0.20 \pm 0.17$ & $0.09 \pm 0.09$ & 0.04 \\
\hline $\mathrm{PCr}$ & $74.8^{\mathrm{ab}} \pm 15.2$ & $81.3^{\mathrm{a}} \pm 32.4$ & $60.0^{c} \pm 20.3$ & $65.0^{b c} \pm 18.4$ & 5.87 \\
\hline ATP & $25.2^{\mathrm{a}} \pm 2.9$ & $24.0^{\mathrm{ab}} \pm 3.7$ & $21.8^{\mathrm{b}} \pm 5.9$ & $23.2^{\mathrm{ab}} \pm 4.4$ & 1.52 \\
\hline ADP & $2.23^{\mathrm{ab}} \pm 0.30$ & $2.27^{\mathrm{ab}} \pm 0.30$ & $2.25^{\mathrm{a}} \pm 0.41$ & $2.16^{b} \pm 0.35$ & 0.12 \\
\hline AMP & $0.12^{\mathrm{a}} \pm 0.04$ & $0.07^{b} \pm 0.04$ & $0.08^{\mathrm{ab}} \pm 0.06$ & $0.11^{a b} \pm 0.04$ & 0.016 \\
\hline
\end{tabular}

Significant differences $(P<0.05)$ are indicated by the superscripts ${ }^{a, b}, c$, meaning no difference in case of an identical superscript letter. Data are expressed as $\mu$ mol/g dry weight $(n=12)$. 
During the exercise tests characteristic changes in the content of some biochemical parameters in skeletal muscle tissue were observed (Table: 7.4). Phosphocreatine levels, ATP, and glycogen content dropped during incremental exercise tests, while IMP and lactate increased. No differences between the four groups could be found.

Table 7.4 Difference in muscle content of a variety of biochemical parameters measured in biopsies taken before and after the exercise test (meantsd and sed). Data obtained in the four groups subjected to different training protocols were pooled.

\begin{tabular}{|c|c|c|c|c|c|}
\hline \multicolumn{6}{|c|}{ Day of the experimental period } \\
\hline & 0 & 40 & 71 & 106 & sed \\
\hline Glycogen & $247 \pm 180$ & $372 \pm 225$ & $354 \pm 225$ & $221 \pm 156$ & 79.6 \\
\hline Lactate & $-86.7^{\mathrm{a}} \pm 36.8$ & $-87.2^{\mathrm{a}} \pm 27.3$ & $-87.8^{\mathrm{a}} \pm 36.6$ & $-53.2^{b} \pm 25.2$ & 12.32 \\
\hline IMP & $-2.46 \pm 1.54$ & $-2.56 \pm 2.42$ & $-1.55 \pm 1.26$ & $-1.46 \pm 1.19$ & 0.56 \\
\hline $\mathrm{PCr}$ & $49.6^{\mathrm{ab}} \pm 18.8$ & $53.4^{\mathrm{a}} \pm 33.6$ & $38.4^{b} \pm 24.2$ & $41.1^{\mathrm{ab}} \pm 22.8$ & 6.83 \\
\hline ATP & $6.56 \pm 5.1$ & $8.16 \pm 7.4$ & $5.36 \pm 7.3$ & $8.51 \pm 7.4$ & 2.73 \\
\hline ADP & $0.03 \pm 0.57$ & $-0.03 \pm 0.53$ & $-0.02 \pm 0.66$ & $0.13 \pm 0.35$ & 0.23 \\
\hline AMP & $-0.02 \pm 0.11$ & $-0.00 \pm 0.07$ & $0.01 \pm 0.08$ & $0.00 \pm 0.06$ & 0.039 \\
\hline
\end{tabular}

Significant differences $(P<0.05)$ are indicated by the superscripts ${ }^{a, b}$, meaning no difference in case of an identical superscript letter. Data are expressed as $\mu$ mol/g dry weight $(n=12)$.

The content of ADP and AMP did not consistently change during the exercise tests. In the pooled data, the decline in tissue glycogen and. ATP during the incremental exercise test was comparable between the different test days. Only the increase in lactate was significantly less in the last exercise test on day 106. The other compounds did not show a consistent change during the experimental period (Table 7.4).

In the groups with additional intensive exercise bouts the increase of IMP in tissue seemed to decline during the course of the experiment (Table 7.5). This effect reached the level of significance in group [3_85_6] when day 71 and 106 was compared with day 0 and 40 . In both groups with 6 additional intensive exercise bouts 
also the increase in tissue lactate was significantly lower on day 106 as compared with day 0 (Table 7.6).

Table 7.5 Difference in IMP content of the muscle biopsies taken before and after the exercise test (meantsd). Data obtained in the four groups subjected to different training protocols are shown.

\begin{tabular}{l|cccc}
\hline & \multicolumn{4}{|c}{ Day of the experimental period } \\
\hline protocol & 0 & 40 & 71 & 106 \\
\hline$\left[3 \_85 \_0\right]$ & $-1.8_{y z} \pm 0.8$ & $-2.7_{x y} \pm 2.1$ & $-1.3 \pm 1.1$ & $-1.6 \pm 0.7$ \\
{$\left[3 \_85 \_6\right]$} & $-4.4^{\mathrm{a}}{ }_{x} \pm 1.5$ & $-4.7^{\mathrm{a}} \pm \mathbf{x} \pm 4.0$ & $-1.9^{\mathrm{b}} \pm 1.4$ & $-1.1^{\mathrm{b}} \pm 0.2$ \\
{$\left[6 \_70 \_0\right]$} & $-1.4_{\mathrm{yz}} \pm 1.4$ & $-1.3_{\mathrm{y}} \pm 0.7$ & $-2.1 \pm 2.0$ & $-2.8 \pm 1.5$ \\
{$\left[6 \_70 \_6\right]$} & $-2.2_{x z} \pm 0.5$ & $-1.5 \mathrm{y} \pm 0.8$ & $-0.9 \pm 0.2$ & $-0.3 \pm 0.3$ \\
\hline
\end{tabular}

Significant differences $(P<0.05)$ are indicated in the horizontal direction by the superscripts $a b$, and in the vertical direction by the subscripts $x, y, z$ meaning no difference in case of an identical letter. Data are expressed as $\mu \mathrm{mol} / \mathrm{g}$ dry weight ( $n=3$ in each group; sed $=1,29)$.

Table 7.6 Difference in lactate content of the muscle biopsies taken before and after the exercise test (mean \pm sd). Data obtained in the four groups subjected to different training protocols are shown.

\begin{tabular}{l|cccc}
\hline & \multicolumn{4}{|c}{ Day of the experimental period } \\
\hline protocol & 0 & 40 & 71 & 106 \\
\hline$\left[3 \_85 \_0\right]$ & $-73.5^{\mathrm{ab}} \pm 34.9$ & $-94.4^{\mathrm{a}} \pm 16.5$ & $-81.7^{\mathrm{ab}} \pm 44.4$ & $-43.8^{\mathrm{b}} \pm 36.4$ \\
{$\left[3 \_85 \_6\right]$} & $-125.9^{\mathrm{a}} \pm 7.7$ & $-97.4^{\mathrm{ab}} \pm 32.5$ & $-86.1^{\mathrm{ab}} \pm 51.9$ & $-54.2^{\mathrm{b}} \pm 20.1$ \\
{$\left[6 \_70 \_0\right]$} & $-55.4 \pm 39.2$ & $-66.7 \pm 37.4$ & $-104.3 \pm 21.7$ & $-75.0 \pm 14.1$ \\
{$\left[6 \_70 \_6\right]$} & $-92.2^{\mathrm{a}} \pm 23.6$ & $-90.5^{\mathrm{a}} \pm 23.3$ & $-78.9^{\mathrm{ab}} \pm 40.4$ & $-39.8^{\mathrm{b}} \pm 21.4$ \\
\hline
\end{tabular}

Significant differences $(P<0.05)$ are indicated in the horizontal direction by the superscripts $a, b, c$, meaning no difference in case of an identical letter. Data are expressed as $\mu$ mol/g dry weight $(n=3$ in each group; $s e d=27.5)$. 


\subsection{DISCUSSION}

Since a previous study (chapter 6) showed that 3 minute bouts of aerobic exercise increases performance capacity, the objective of the present investigation was to study whether six or three 3-min bouts of aerobic exercise at 70 and $85 \%$ of the peak heart rate, respectively, combined with an anaerobic component performed as. six 1-min sprints, could further improve the performance capacity in horses.

Heart rate recordings showed that the intensity of the aerobic work bouts in the different groups corresponded well with the target intensities of $70 \%$ and $85 \%$ of the peak heart rate. Heart rate levels at the end of the 1-min sprint intervals proved to be well below the maximal possible heart rates. Because the horses appeared to be fatigued after the exercise including six 1 -min sprints, the training intensity was not increased further. Since plasma lactate levels were available a few weeks after the exercise was performed this parameter could not be used during the training in determining the training intensity.

The mean plasma lactate levels at the end of the training bout, which varied between 7 and $8 \mathrm{mM}$, indicate an anaerobic contribution to energy delivery during the interval training. However, compared to the levels seen at exhaustion (ranging from $17.0 \pm 2.1$ to $32.7 \pm 2.3 \mathrm{mM}$ ) the average plasma lactate levels during training must be regarded as moderate.

None of the 4 training protocols was able to induce significant changes in performance capacity. It should be noted that a considerable fluctuation in a range of $2-5 \%$ in total running time could be observed within groups at different time points. These changes did not coincide with changes in blood chemical parameters, such as creatine kinase, alanine aminotransferase, aspartate aminotransferase and lactate dehydrogenase, or biochemical markers in muscle tissue. At present, no feasible explanation for the fluctuations can be offered. It has been shown that also in men unexplained spontaneous fluctuations in performance occur, whereas the range of the fluctuations was comparable (9). Therefore, the present data indicate that the variability of performance in horses is similar to that in men. 
One of the explanations for the absence of consistent changes in performance capacity could be that the horses were not really untrained at the onset of the experiment. Until 2 months before the start of this experiment the horses were used for another training experiment. At the end of the previous study the mean total running time until exhaustion and the mean total amount of vertical work were $768 \mathrm{~s}$ and $1406 \mathrm{~kJ}$, respectively. These values were not significantly different from the performance parameters measured at day 0 in the present study. In the present study duration and intensity of the training exercise proved to be insufficient to induce an improvement of the physical performance capacity, It is feasible that exercise at a higher intensity and/or of a longer duration is required to induce a further improvement of performance capacity. However, it cannot be excluded that the maximal performance capacity for each individual horse had already been reached due to the training in the previous study.

It is interesting to note that between the end of the study described in chapter 6 and the start of the present exercise protocol, the performance capacity did not decrease significantly. So, in these horses a period of about 2 months of low-intensity exercise had no adverse effect on their performance capacity. This seems to be in line with the results of Erickson et al. (3) who found no changes in heart rate and plasma lactate levels at a given speed after a period of detraining and retraining. Also Foreman et al. (5) found no change in plasma lactate levels after a 5-week period of detraining. Both investigators used horses that were trained before.

With respect to the content of biochemical parameters in biopsies obtained from the gluteus medius muscle no major changes were found in any training protocol at the different time points. When the data of the 4 protocols are pooled and compared at different time points the conclușion can be drawn that glycogen levels increased during the training protocol. This increase indicates an adaptation of the intracellular carbohydrate store to exercise training. No consistent change in the tissue content of the other biochemical parameters was observed throughout the training period. The tissue content of glycogen significantly decreased during the incremental exercise test, indicating a substantial contribution of the endogenous carbohydrate 
store to the energy converting processes. The observation that the amount of glycogen used was independent of test day and exercise protocol indicates that this particular part of skeletal muscle energy metabolism is not influenced by the training protocols as applied in the present study. Moreover, the decline of phosphocreatine and ATP during the exercise test was also independent of test day and exercise protocol. The latter findings suggest that the energy deficit is comparable between the various protocols applied. Taking into account that running time till exhaustion and amount of vertical work did not change either, the conclusion can be drawn that overall chemical energy conversion did not improve as a consequence of the various training protocols. In this respect it is of interest to note that in the two groups with six additional intensive exercise bouts during the training session, the increase in tissue lactate and IMP was found to be less at day 106 than at the start of the experiment. This finding can be explained in two ways: either less lactate and IMP is produced or lactate is more rapidly released to the extracellular space and IMP more efficiently metabolised to (oxy)purines, which are in turn released to the interstitial and vascular compartment. At present no choice can be made between the two alternative explanations. Since both lactate and IMP exert an adverse effect on muscular contractile function (7), a less pronounced accumulation of the two metabolic compounds may be beneficial for performance capacity during a strenuous exercise bout. However, the earlier mentioned findings on running till exhaustion and amount of vertical work do not favour the notion that a lower level of lactate and IMP has great impact on muscle function under the present experimental conditions.

In conclusion, the results of this study suggest that training protocols consisting of aerobic intervals combined with six 1-min sprints resulting in plasma lactate levels of 7-8 $\mathrm{mM}$, are not sufficient to realise a further increased performance capacity. However, as a result of a previous study, the horses were relatively well trained at the start of this experiment. The moderate exercise intensity is probably the most important factor in explaining the lack of significant changes. So, in practical horse training, exercise of long duration and of an intensity resulting in plasma lactate levels below $10 \mathrm{mM}$ is most likely ineffective in improving the metabolic capacity. Exercise of 
higher intensity, resulting in plasma lactate levels above $10 \mathrm{mM}$, will be necessary to realise the maximal performance capacity of horses (see also chapter 5).

\subsection{REFERENCES}

1. Asstrand P.O., K. Rodahl. Textbook of Work Physiology. Physiological bases of exercise. McGraw-Hill Book Company, New York, 1986.

2. Bergstrōm J.. Percutaneous needle biopsy of skeletal muscle in physiological and clinical research. Scand J Clin Invest 35: 609-616, 1975.

3. Erickson H.H., W.L. Sexton, B.K. Erickson, J.R. Coffman. Cardiopulmonary response to exercise and detraining in the Quarter horse. In: Gillespie I.R., N.E. Robinson (eds): Equine Exercise Physiology 2 Edition, Edwards Brothers, Ann Arbor, USA, 1987, pp. 41-51.

4. Essen-Gustavsson B., D. McMiken, K. Karlstrom, A. Lindholm, S.G.B. Persson, J. Thornton. Muscular adaptation of horses during intensive training and detraining. Eq Vet / 21: 27-33, 1989.

5. Foreman J.H., W.M. Bayly, B.D. Grant, P.D. Gollnick. Standardized Exercise Test and Daily Heart Rate Responses of Thoroughbreds Undergoing Conventional Race Training and Detraining. Am J Vet Res 51: 914-920, 1990.

6. Genstat 5 Committee (R.W. Payne, chairman, P.W. Lane, secretary). Design and analysis of experiments. In: Genstat 5 Release 3 Reference Manual, Clarendon Press, Oxford, 1993, pp. $461-538$.

7. Haan A. de. Muscle metabolism during fatiguing exercise. In: Sargeant \& Kernell (eds): Neuromuscular fatigue, North Holland, Amsterdam, Oxford, New York, Tokyo, 1993, pp. 16-23.

8. Knight P.K., A.K. Sinha, R.J. Rose. Effects of training intensity on maximum oxygen uptake. In: Persson S.G.B., A. Lindholm, L.B. Jeffcott (eds): Equine Exercise Physiology 3, Almqvist \& Wikself Tryckeri, Uppsala, 1991, pp. 77-82.

9. Kuipers H., F. Verstappen, H. Keizer, P. Geurten, and G. van Kranenburg. Variability of aerobic performance in the laboratory and its physiological correlates. Int I Sports med 6: $197-201,1985$.

10. Lindholm A., K. Piehl. Fibre composition, enzyme activity and concentrations of metabolites and electrolytes in muscles of standardbred horses. Acta Vet Scand 15: 287-309, 1974.

11. Lindholm A., B. Saltin. The physiological and biochemical response of standardbred horses to exercise of varying speed and duration. Acta Vet Scand 15: 310-324, 1974. 
12. Lindholm A., H. Bjerneld, B. Saltin. Glycogen depletion pattern in muscle fibre of trotting horses. Acta Physiol Scand 90: 475-484, 1974.

13. McArdle W.D., F.I. Katch, V.L. Katch. Training for anaerobic and aerobic power. In: Exercise Physiology. Energy, nutrition and human performance: 347-370, Lea \& Febiger, Philadelphia, 1986.

14. McKeever K.H., W.A. Schurg, S.H. Jarrett, V.A. Convertino. Exercise training-induced hypervolemia in the horse. Med Sci Sports Exerc 19: 21-27, 1987.

15. Milne D.W., A.A. Gabel, W.W. Muir, R.T. Skarda. Effect of training on heart rate, cardiac output, and lactic acid in standardbred horses using a standardized exercise test. J Eq Med Surg 1: 131-135, 1977.

16. Rose R.J., J.R. Allen, D.R. Hodgson, J.H. Stewart, W. Chan. Responses to submaximal treadmill exercise and training in the horse: Changes in haematology, arterial blood gas and acid base measurements. Vet Rec 113: 612-618, 1983.

17. Thornton J., B. Essen-Gustavsson, A. Lindholm, D. McMiken, S.G.B. Persson. Effects of Training and Detraining on Oxygen Uptake, Cardiac Output, Blood Gas Tensions, $\mathrm{PH}$ and Lactate Concentrations during and after Exercise in the Horse. In: Snow D.H., S.G.B. Persson, R.J. Rose (eds): Equine Exercise Physiology, Burlington Press, Cambridge, 1983, pp. $470-486$. 
EFFECTS OF THREE AND SIX 1-MIN ANAEROBIC

EXERCISE BOUTS, ADDED TO CONTINUOUS

AEROBIC TRAINING, ON THE PERFORMANCE

CAPACITY OF HORSES

This chapter will be published as:

G. Bruin, H. Kuipers, G. André, and G.J. van der Vusse

Effects of three and six 1-min anaerobic exercise bouts, added to continuous aerobic training, on the performance capacity of horses

Int J Sports Med (Accepted for publication) 


\subsection{ABSTRACT}

Twelve two-year-old Standardbred trotter stallions were used to investigate the effect of 3 or 6 anaerobic exercise bouts, added to continuous aerobic exercise, on performance capacity, as determined in an incremental exercise test on a treadmill.

The horses were randomly assigned to three groups. Group 1 performed continuous aerobic exercise at an intensity of $60 \%$ of the peak heart rate. Group 2 performed in addition to the continuous exercise three 1-min anaerobic sprints at an intensity of $>90 \%$ of the peak heart rate, and group 3 performed in addition to the continuous exercise six 1-min sprints at an intensity of $>90 \%$ of the peak heart rate. The 1-min sprints were interspersed with 4-min periods of exercise at $60 \%$ of the peak heart rate. Total exercise time was $26 \mathrm{~min}$ for all groups.

Every third week the horses performed an incremental exercise test on a treadmill until exhaustion. Changes in the performance capacity were evaluated from possible changes in the regression equations of heart rate versus power output, and of plasma lactate versus power output, in the total running time, and in the total amount of vertical work.

During the first 6 weeks a significant increase in total running time and total amount of vertical work, was attained in all groups regardless of the number of anaerobic exercise sprints performed per work bout during the training sessions. The increase of performance capacity lasted until the end of the experimental period $1 \sim 15$ weeks) only in the group with 6 sprints. Irrespective of the number of intensive exercise bouts, a lower plasma lactate at a given power output was seen after the training period. No changes in the relationship between heart rate and power output were observed.

Skeletal muscle glycogen levels transiently increased during the training period. The tissue content. of phosphocreatine at rest showed a steady decline. During the 
Chapter 8

exercise test no consistent changes were found in the changes of energy-rich phosphates and related compounds.

In conclusion, endurance training at $60 \%$ of the peak heart rate for $42-64$ days increased performance capacity in an exercise test. Adding three or six 1-mir exercise bouts at $>90 \%$ of the peak heart rate to the endurance training did not further increase exercise capacity. However, the data of the present study suggest that in order to maintain a higher level of fitness for up to 106 days endurance training only or with three high intensity 1-min bouts per session was not sufficient. Six additional periods of 1-min duration were required to maintain the work capacity at the acquired level. 


\subsection{INTRODUCTION}

In horse races, which commonly last between 2 and $4 \mathrm{~min}$, muscle energy conversion relies on both aerobic and anaerobic metabolism $(2,8$, 9). The training intensity is an important factor for improving both aerobic and anaerobic performances. For improvement of the aerobic capacity exercise with an intensity $z$ $70 \% \mathrm{VO}_{2 \max }$ should be included in the training program (9). Anaerobic training requires exercise with an intensity varying from $>90 \%$ of the maximal heart rate to allout exercise (9). Therefore, training programs for horses participating in $2-4 \mathrm{~min}$ contests preferably includes also anaerobic exercise (8).

Anaerobic training can be performed either in one single, short, race-like type of exercise, resulting in fatigue within 2-3 min, or in repeated bouts of heavy, near exhaustive, exercise of shorter duration, usually referred to as interval training. By using interval training the total amount of high-intensity exercise until fatigue can be increased (9).

In horses, neither the optimal number of high intensity exercise bouts nor the optimal exercise intensity for a maximal improvement of performance capacity has been determined experimentally. In a previous study (chapter 7) it was found that intermittent aerobic exercise combined with 6 anaerobic sprints was not able to increase performance capacity. However, this may have been attributed to the relatively high state of training at the beginning of the experiment. Therefore in the present study horses with a low training status were used. The aim of this investigation was to study the effect of low intensity endurance training $(60 \%$ of the peak heart rate) to which three or six high-intensity 1-min bouts are added. Endurance capacity was measured in a incremental exercise test on the treadmill. To obtain insight into the physiological basis of possible changes in performance capacity, as determined in the incremental exercise test, a number of physiological and biochemical variables was measured, such as plasma volume, red blood cell volume and, in muscle biopsies, glycogen, lactate, energy-rich phosphates and related compounds (12). 


\subsection{MATERIAL AND METHODS}

\section{$\underline{8.3 .1}$ Horses and training}

Twelve 2-year-old Standardbred trotter stallions entered the study after being housed at the experimental farm for a period of almost 1 year. During this year the horses were accustomed to the treadmill and the rotary exerciser, while only exercise with an intensity below $60 \%$ of the peak heart rate was performed. In this sense the horses can be considered as untrained. In a randomised block design the horses were assigned to one of three different training protocols. Heart rate was used to determine the exercise intensity. The exercise intensity was based on fixed percentages of the peak heart rate. The peak heart rate was the maximal heart rate ever measured in the individual horse under investigation during an exercise test until exhaustion.

The three different training protocols consisted of:

1. group SO: endurance exercise at an intensity of $60 \%$ of the peak. heart rate during 26 min (Fig. 8.1);

2. group $53: 3$ bouts of $1 \mathrm{~min}$ at $>90 \%$, interspersed with $4 \mathrm{~min}$ at $60 \%$ of the peak heart rate; after the last sprint exercise at an intensity of $60 \%$ of the peak heart rate was continued until a total of $26 \mathrm{~min}$ of exercise was completed (Fig. 8.2):

3. group S6: 6 bouts of $1 \mathrm{~min}$ at $>90 \%$ interspersed with 4 min at $60 \%$ of the peak heart rate (Fig. 8.3).

Each training session was preceded by a 6 -min warm-up at $4.5 \mathrm{~m} / \mathrm{s}$. The three different training protocols were performed 3 times a week for a period of 106 days on a treadmill with an inclination of $6.25 \%$. In the afternoon of the days with treadmill exercise the horses performed also an exercise bout of $30 \mathrm{~min}$ at $1.5 \mathrm{~m} / \mathrm{s}$ in a rotary exerciser. The days with treadmill training were interspersed with days on which only endurance running at an intensity between $40 \%$ and $60 \%$ of the peak heart rate was performed in a rotary exerciser. The horses were exercised 6 days per week with 1 day rest. 


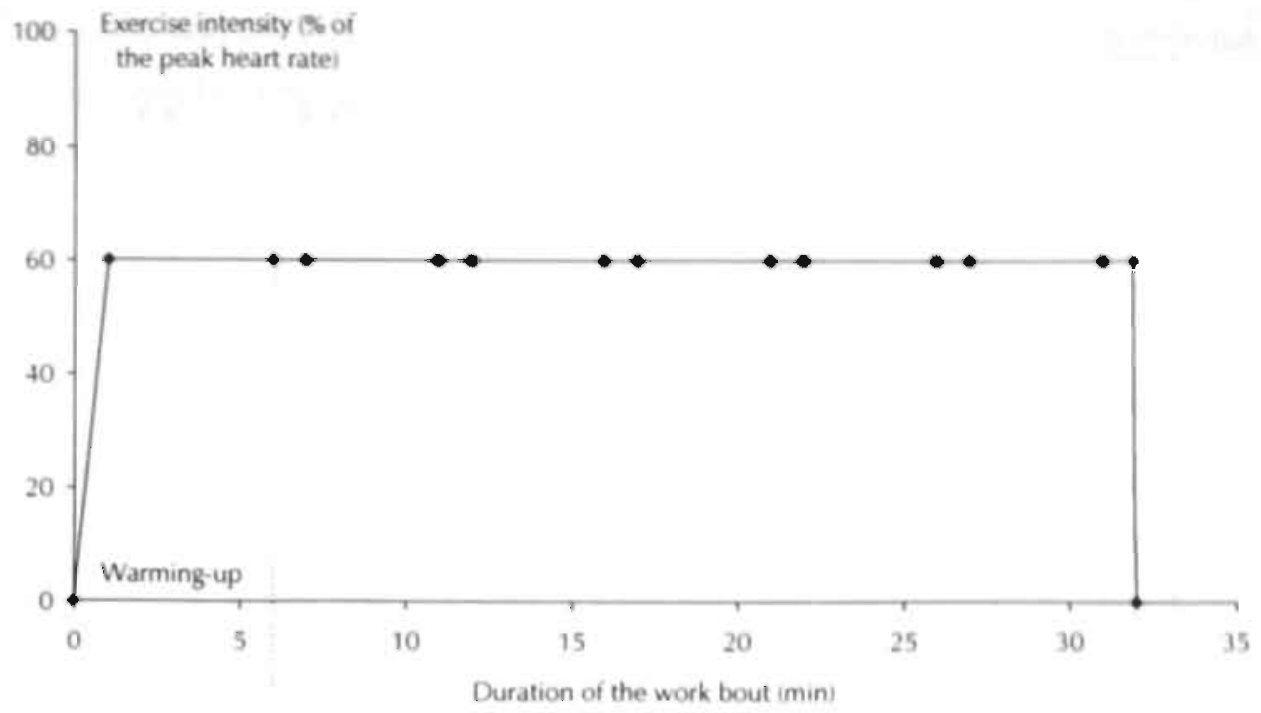

Fig. 8.1 Exercise protocol of group SO. The horses performed only endurance exercise at an intensity of $60 \%$ of the peak heart rate during a period of $26 \mathrm{~min}$.

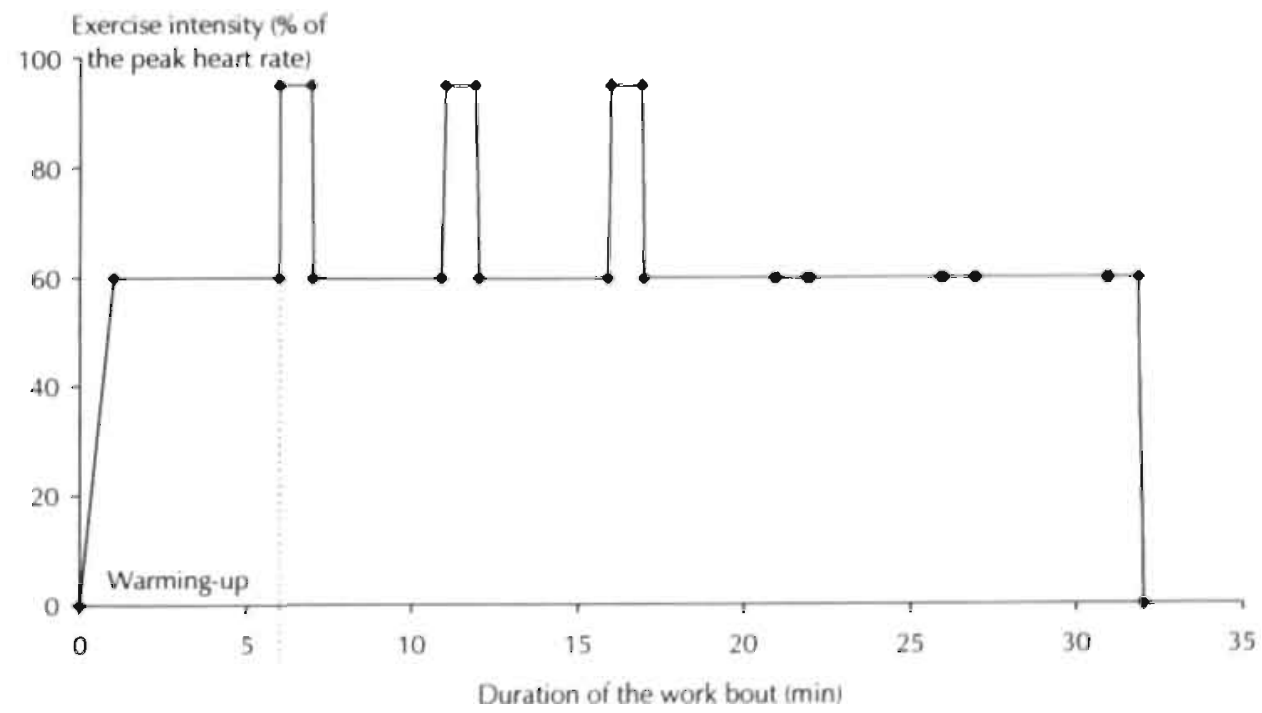

Fig. 8.2 Exercise protocol of group 53. This group performed three 1-min sprints with endurance exercise at an intensity of $60 \%$ of the peak heart rate till a total duration of the work bout of 26 min. The sprints were interspersed with 4 -min endurance exercise at an intensity of $60 \%$ of the peak heart rate. 


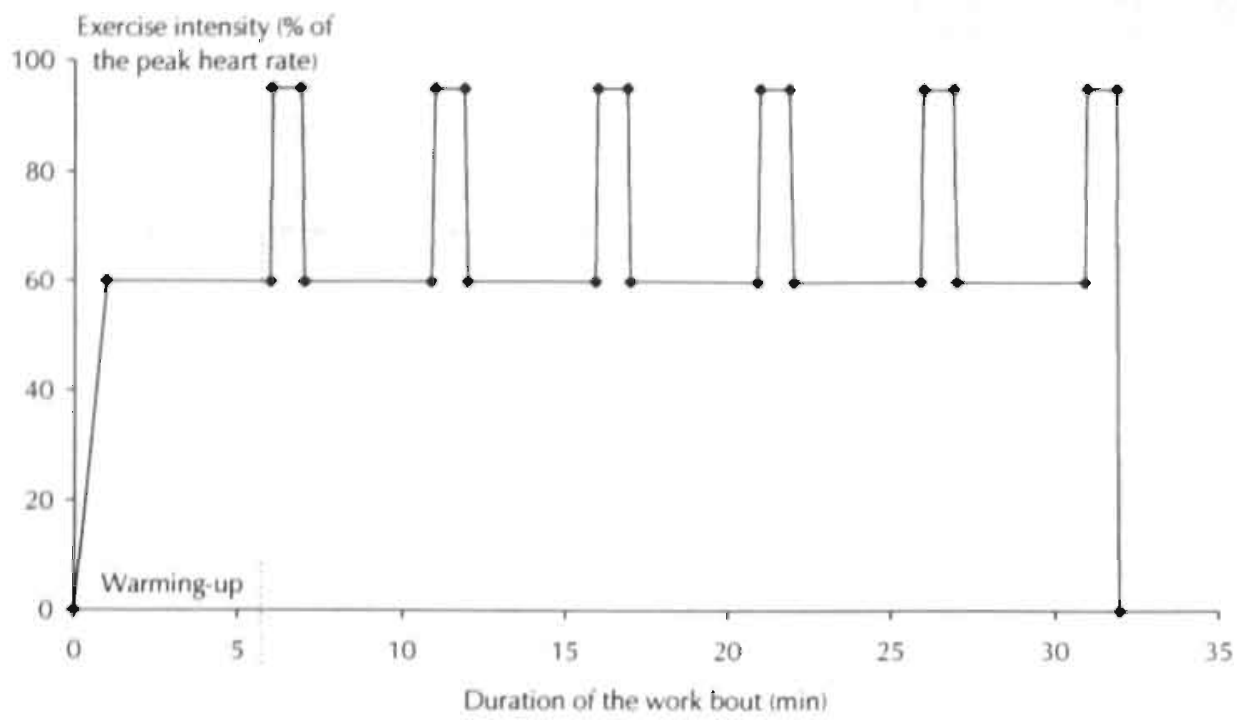

Fig. 8.3 Exercise protocol of group S6. This group performed six 1-min sprints with a total duration of the work bout of $26 \mathrm{~min}$. The sprints were interspersed with 4-min endurance exercise at an intensity of $60 \%$ of the peak heart rate.

Once a week the speed of the treadmill during the interval bouts and endurance exercise was adjusted according to the results of training and tests in order to obtain the desired level of the peak heart rate. The horses were fed according to the energy requirements and were individually housed.

Every day at 8 a.m. body weight was measured. On training days the horses were prepared by attaching the safety belt and the belt for the heart rate monitor. Two and five mins after stopping the training a blood sample was taken to determine the plasma lactate concentration.

\subsubsection{Incremental exercise test}

On test days the horses were catheterised and a muscle biopsy was taken from the gluteus medius muscle prior to the exercise test $(3,4,8)$. The safety belt and the belt for the heart rate monitor were attached and, when the horse was standing on the 
treadmill, also the ecg-equipment was attached to the belt of the heart rate monitor. Blood samples were taken prior to the exercise test, during the last $15 \mathrm{~s}$ of each step and 2 and $5 \mathrm{~min}$ after finishing the test. Immediately after the test $15 \mathrm{ml} 2 \%$ Evan's Blue dye was injected (thereafter the catheter was washed with $5 \mathrm{ml}$ physiologic saline solution), a second muscle biopsy was taken and the rectal temperature was measured. After that, the horse got a shower, was weighed and put into the housing box. Fifteen mins after the Evan's Blue injection a blood sample was taken to determine the plasma volume. The tests were performed between 7 a.m. and noon.

Six incremental exercise tests were done on the treadmill during the experimental period (day 1, 21, 42, 64, 84 and 106). The incremental exercise tests were performed after 1 day of rest. After a 6 -min warming-up at $4.5 \mathrm{~m} / \mathrm{s}$, the incremental exercise tests consisted of a 2 min step at a treadmill speed of $2 \mathrm{~m} / \mathrm{s}$. Thereafter the speed was increased to $5 \mathrm{~m} / \mathrm{s}$ and every two min further increased with $1 \mathrm{~m} / \mathrm{s}$ until exhaustion. Exhaustion was considered to be reached when, despite encouragement, the horse was not able to maintain the running speed.

\subsubsection{Data collection}

During training exercise and during the incremental exercise test the heart rate was continuously recorded every 5 s by means of the "Sport tester PE3000" (Polar Electro) and during the incremental exercise tests also by means of an electrocardiogram (ecg) (Schwarzer CS6000). During the incremental exercise test time to exhaustion was measured. Since the treadmill had a $6.25 \%$ incline, the power output and the total amount of vertical work $(\mathrm{kJ})$ delivered until exhaustion could be calculated.

The power output (Watt) is calculated according to the formula:

$$
\text { power }=9.81 \times \text { body weight } \times \text { speed } \times \sin \alpha
$$

In this formula body-weight is expressed in $\mathrm{kg}$, speed in $\mathrm{m} / \mathrm{s}$, and $\alpha$ is the angle (degrees) in inclination of the treadmill. 
The amount of vertical work (kJ) per stage is calculated according to the formula:

$$
\text { work }=9.81 \times \text { body-weight } \times \text { speed } \times \text { duration } \times \sin \alpha
$$

in which duration is the running time (s) at each treadmill speed. The amount of work. at each speed is summated, resulting in the total amount of work. Only the vertical component was included.

The relationship between heart rate and power output is described by the linear relation $(\mathrm{HR}=$ heart rate in beats $/ \mathrm{min} ;$ power $=$ power output in Watt; $\beta$ ${ }_{0}=$ intercept;

$\beta_{1}=$ regression coefficient):

$$
H R=\beta_{0}+\beta_{1} \times \text { power }
$$

The relationship between plasma lactate concentration $(\mathrm{mM})$ and power output (Watt) is described by the exponential curve:

$$
\text { lactate }=A+B \times R^{\text {power }}
$$

in which lactate is the plasma lactate concentration $(\mathrm{mM}), \mathrm{A}, \mathrm{B}$ and $\mathrm{R}$ are regression parameters, and power is the power output (Watt).

Changes in the performance capacity were measured as changes in the regression of heart rate (beats/min) versus power output (Watt), plasma lactate (mM) versus power output (Watt), the total running time until exhaustion (s), and the total amount of vertical work until exhaustion ( $k J)$.

\subsubsection{Measurement of plasma lactate and muscle enzymes}

The blood samples were centrifuged in heparinized Eppendorf-cups at 12,000 revolutions per $\min (8800 \mathrm{~g})$. The plasma was immediately frozen at $-20^{\circ} \mathrm{C}$ and subsequently stored at $-80^{\circ} \mathrm{C}$. Plasma lactate was determined with a Lactate analyser 640 (La Roche). The activity of muscle or liver enzymes (CK, AST, ALT, LDH) in blood plasma was determined as indicated in chapter 5 . 
8.3.5 Measurement of plasma volume and red blood cell volume

The blood sample was centrifuged during $10 \mathrm{~min}$ at 2,000 revolutions per min. The resulting plasma was centrifuged again during $30 \mathrm{~min}$ at 12,000 revolutions per $\min$ and frozen at $-20^{\circ} \mathrm{C}$. The frozen samples were stored at $-80^{\circ} \mathrm{C}$. The plasma volume was calculated according to:

$$
P V=\frac{E B}{C}
$$

where PV is the plasma volume in $\mathrm{ml}$, EB the amount of injected Evans blue dye in $\mathrm{mg}$, and $\mathrm{C}$ the concentration of the dye in $\mathrm{mg} / \mathrm{ml}$ plasma.

The normalised red blood cell volume is:

$$
C V=\frac{P V \times H c t}{(100-H c t) \times \text { bodyweight }}
$$

where $\mathrm{CV}$ is the red blood cell volume in $\mathrm{ml} / \mathrm{kg}$, PV the plasma volume in $\mathrm{ml}$ and $\mathrm{Hct}$ the venous hematocrit (\%). Body weight is expressed in $\mathrm{kg}$.

\subsubsection{Measurement of energy-rich phosphates, glycogen and lactate}

The metabolic status of the muscular tissue was judged by determination of the amount of phosphocreatine ( $\mathrm{PCr}$ ), adenosine triphosphate (ATP), adenosine diphosphate (ADP), adenosine monophosphate (AMP), and inosine monophosphate (IMP) in biopsies obtained from the gluteus medius muscle. Also the tissue content of glycogen and lactate was determined.

The muscle tissue from the biopsy was rapidly frozen in liquid nitrogen. Time interval between sampling of tissue and freezing was in the order of 5-10 s. The frozen tissue samples were stored at $-80^{\circ} \mathrm{C}$. Prior to biochemical analysis the samples were freeze-dried at $-30^{\circ} \mathrm{C}$ overnight. The content of $\mathrm{PCr}$, adenine nucleotides and related compounds, glycogen, lactate and glucose was determined as described earlier (11, $13,14,16)$. Details of the analytical techniques are given in chapter 6 . 


\subsubsection{Statistics}

The experiment was performed in a completely randomised block design. Blocks were based on performance capacity immediately before the start of the experiment. Analysis was performed, using the Genstat package (6), as described in detail in chapter 6. Differences were regarded significant at $\mathrm{P}<0.05$.

\subsection{RESULTS}

\subsubsection{Acceptance of the exercise protocol and training intensity}

The horses accepted the training protocol very well. One horse could not sustain the training protocol because of lameness due to osteorthritis, probably enhanced by a fracture in early life as a foa! (group S6). The other horses all performed an equal number of training sessions on the treadmill, except one horse which could not perform 4 sessions because of suspected lameness (group S3). No serious health problems were observed during the experimental period. The plasma activity of creatine kinase, aspartate aminotransferase, alanine aminotransferase and lactate dehydrogenase proved to be within normal ranges and were without systematic changes (data not shown).

Analysis of heart rate recorded during training revealed that the horses actually reached $95 \%$ and $96 \%$ of the peak heart rate at the end of the 1-min sprint exercise bouts in the groups with 3 and 6 sprints, respectively.

Lactate levels in blood plasma, obtained immediately after the exercise bouts on training days, showed an average of $2.6 \pm 1.1,8.8 \pm 3.5$ and $9.6 \pm 3.6 \mathrm{mM}$ in the groups without sprints, and with 3 and 6 sprints, respectively.

\subsubsection{Incremental exercise tests}

Performance capacity, expressed as total running time until exhaustion, increased transiently in group S0 and S3 during the training period (Table 8.1). The group with only endurance running ( $\mathrm{SO}$ ) and the group with endurance running, plus 6 intensive exercise bouts (S6) showed a significant increase during the course of the 
training period from day 42 on. The highest absolute values were observed during the incremental exercise tests performed on day 64,64 , and 84 in the groups with 0,3 , and 6 sprints, respectively. In the groups without sprints and with 3 sprints the values of running time until exhaustion returned to pre-training values thereafter. In the group with 6 sprints the value of this parameter remained at the elevated level until the end of the training period (day 106).

Comparable findings were obtained during the incremental exercise tests with the exercise performance parameter total vertical work until exhaustion with a significant increase on day 42 in the groups without and with 6 sprints (Table 8.2). The highest increase during the incremental exercise tests was observed in these groups on day 64 . Thereafter, total vertical work until exhaustion returned to values observed on day 0 of the training protocol in the groups without and with 3 sprints. In the group with 6 sprints, work until exhaustion remained at a higher level than on day 0 . The group with 3 sprints demonstrated a transient increase on day 42 and day 64 , which did not reach the level of significance. On day 84 and 106 a significant difference was present between the groups with 6 and 3 sprints (Table 8.2, Fig. 8.4).

When heart rate during each step of the incremental exercise test was plotted against power output no change in the relationship between the two parameters was observed. This was the case for all three training protocols (data not shown).

When the plasma lactate levels were plotted as a function of power output measured during the incremental exercise test, a lower plasma lactate level at a given power output was observed during the training period, irrespective of the number of intensive exercise bouts applied during the training protocol (Fig. 8.5, 8.6 and 8.7).

During the training period, plasma volume expanded significantly (Table 8.3). There were no significant differences between the three groups under investigation. Red blood cell volume increased during the first three weeks of the training period in the groups without and with 6 sprints (Table 8.4). Thereafter it returned to values not significantly different from values measured on day 0 . No differences between the three groups of horses were observed. 
Jable 8.1 Iotal running time until exhaustion, medsured in the incremental exercise tests at various time intervals, during the training period.

\begin{tabular}{|c|c|c|c|c|c|c|}
\hline & \multicolumn{6}{|c|}{ Day of the experimental period } \\
\hline & 0 & 21 & 42 & 64 & 84 & 106 \\
\hline group S0 & $727^{4} \pm 45$ & $751^{4} \pm 65$ & $782^{t} \pm 62$ & $785^{k} \pm 67$ & $774^{l} \pm 56$ & $740^{.16}{ }_{\mathrm{ky}} \pm 73$ \\
\hline group $\$ 3$ & $753^{d i} \pm 69$ & $753^{a d} \pm 47$ & $773^{x} \pm 51$ & $783^{x} \pm 90$ & $740^{1} \pm 66$ & $722^{\mathrm{b}} \times \pm 55$ \\
\hline group $\$ 6$ & $716^{4} \pm 31$ & $764^{\mathrm{l}} \pm 7$ & $790^{1 /} \pm 39$ & $796^{14} \pm 50$ & $801^{1} \pm 71$ & $792^{\mathrm{b}}{ }^{4}+51$ \\
\hline
\end{tabular}

Significant differences $(P<0.05)$ are indicated in the horizontal direction by the superscripts a,be, meaning no difference in case of an identical letter. Data (mean \pm sd) are expressed as seconds ( $n=4$ in each group; sed $=21.6 / 34.1$ within and between groups, mppertively). 
lable 8.2 Total amount of vertical work until exhaustion, measured in the incremental exercise tests, during the training pernod.

\begin{tabular}{|c|c|c|c|c|c|c|}
\hline & \multicolumn{6}{|c|}{ Day of the experimental period } \\
\hline & 0 & 21 & 42 & 64 & 84 & 106 \\
\hline group 50 & $1297^{d} \pm 98$ & $1337^{a f l} \pm 99$ & $1419^{14} \pm 73$ & $1431^{h} \pm 87$ & $1380^{2 \mathrm{ab}} \times y^{ \pm}+62$ & $1303^{3} \mathrm{xy} \pm 107$ \\
\hline group $\$ 3$ & $1287^{d b^{b}} \pm 170$ & $1314^{\text {abl }} \pm 107$ & $1366^{d} \pm 145$ & $1379+ \pm 196$ & $1259^{6} \times \pm 175$ & $1223^{b}, \pm 107$ \\
\hline group $\mathbf{\$} 6$ & $1308^{a} \pm 80$ & $1423^{a f i} \pm 40$ & $1488^{l} \pm 128$ & $1523^{h} \pm 138$ & $1515^{h} y \pm 198$ & $1495^{6} y+147$ \\
\hline
\end{tabular}

Significant differences $(P<0.05)$ are indicated in the horizontal direction by the superscripts abs, and in the vertical dirertican by the sulsur ripts x,y, meaning no difference in case of an identical letter. Data (mean $\pm s d$ ) are expressed as kilojoules $(n=4$ in rach group: sed = $=55.6 / 97.2$ within and between groups respectively). 
Iable 8.3 Plasma volume (PV), measured immediately after the incremental exercise test.

\begin{tabular}{|c|c|c|c|c|c|c|}
\hline & \multicolumn{6}{|c|}{ Day of the experimental period } \\
\hline & 0 & 21 & 42 & 64 & 84 & 106 \\
\hline group 50 & $22.1 \pm 1.2$ & $21.9^{4} \pm 1.6$ & $21.9^{4} \pm 2.1$ & $22.9^{+1 / 4} \pm 2.8$ & $23.6^{h} \pm 2.6$ & $24.4^{\mathrm{t}} \pm 1.8$ \\
\hline group 53 & $20.5^{ \pm}+2.3$ & $21.7^{\text {aht }} \pm 1.8$ & $22.3^{1 \times 4} \pm 2,1$ & $23.5^{\prime d}+2.7$ & $23.8^{\prime} \pm 2.1$ & $24.1^{\prime} \pm 1.3$ \\
\hline group 56 & $24.6^{4} \pm 0.3$ & $24.0^{4} \pm 0.9$ & $23.4^{4} \pm 1.3$ & $26.0^{1} \pm 1.3$ & $27.0^{1 *}+2.2$ & $27.9^{\prime} \pm 1.6$ \\
\hline
\end{tabular}

Significant ditferences $(P<0.05)$ are indicated in the horizontal direction by the superscripts atb. , meaning no diflerence in calse of an identis al letter. Data (mean. \pm sd) are expressed as litres $(n=4$ in each group; sed $=0.69 / 1.57$ within and between groups respectivily). No statistical differences could be obesenved between the three different groups on corresponding test days. 
table 8. 4 Red blood cell volume (CV), measured immediately after ithe incremental exerciaf :f:

\begin{tabular}{|c|c|c|c|c|c|c|}
\hline & \multicolumn{6}{|c|}{ Day of the experimental period } \\
\hline & 0 & 21 & 42 & 64 & 84 & 106 \\
\hline group 50 & $66.9^{9} \pm 3.1$ & $74.0^{4} \pm 4.9$ & $67.9^{1 x} \pm 5.0$ & $72.3^{\text {al }}+2.9$ & $73.6^{4} \pm 1.1$ & $68.9^{4 i=} \pm 4.1$ \\
\hline group 53 & $66.9 \pm 4.2$ & $70.7 \pm 2.9$ & $69.2 \pm 4.6$ & $69.9 \pm 1.7$ & $70.4 \pm 3.9$ & $683+5,0$ \\
\hline group $\$ 6$ & $66.9^{\mathrm{lx}} \pm 5.4$ & $74.3^{4} \pm 11.0$ & $64.3^{h} \pm 8.1$ & $68.1^{1=} \pm 4.7$ & $71.1^{=1+4.4}$ & $70.9^{\prime \prime \prime}+2.4$ \\
\hline
\end{tabular}

Significant differences $(P<0.05)$ are indicated in the horizontal direction by the superxcripts abir, meaning no difterence in case uf an identical letter. Data (mean values and sdi) are expressed as $\mathrm{ml} / \mathrm{kg}(\mathrm{n}=4$ in each group; $\mathrm{sed}=2.6 \mathrm{~J} / 3.24$ withen and between groups respecively). No statistical differences could be observed between the three different groups on corresponding test days. 
Chapter 8

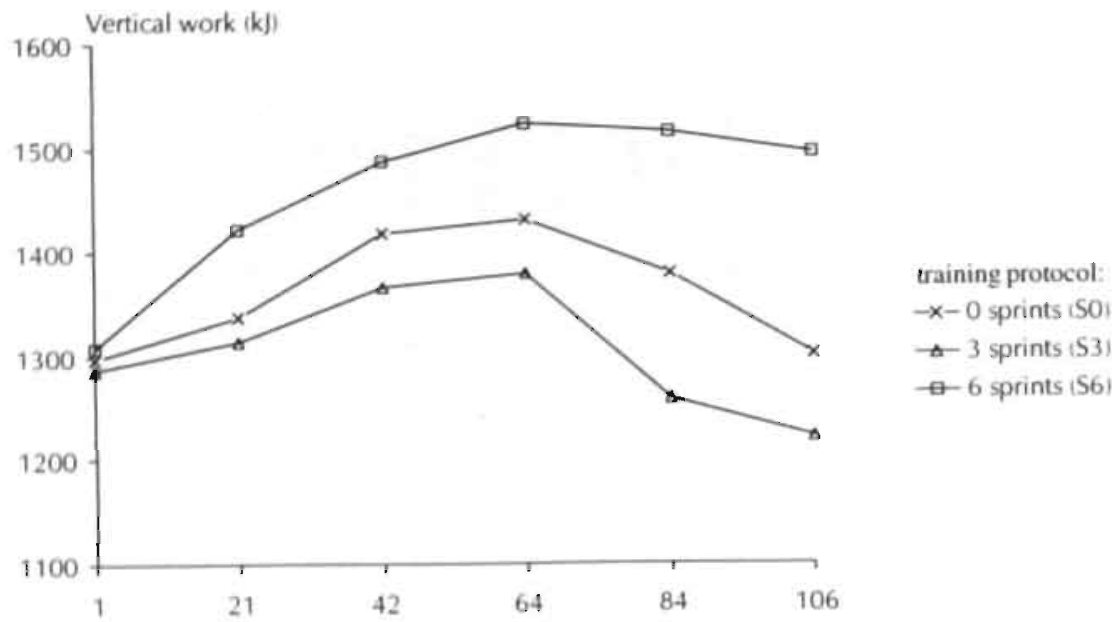

Day of the experiment

Fig. 8.4 Mean amount of vertical work, until exhaustion for the experimental groups at the different test days. The values are expressed as kilojoules $(k j)$. The group with 3 sprints demonstrated a transient increase on day 42 and day 64, which did not reach the level of significance. On day 84 and 106 a significant difference was present between the groups with 6 and 3 sprints.

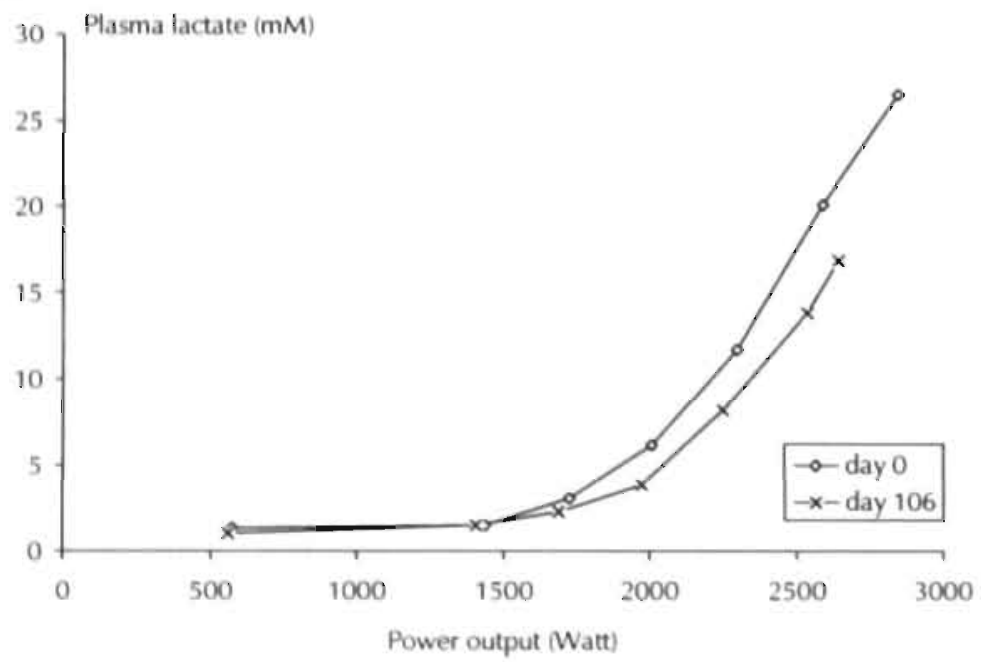

Fig. 8.5 Mean plasma lactate levels of the horses in group SO at day 0 and day 106. The difference between test days proved to be significant (significant different regression parameters to fit the relationship). 


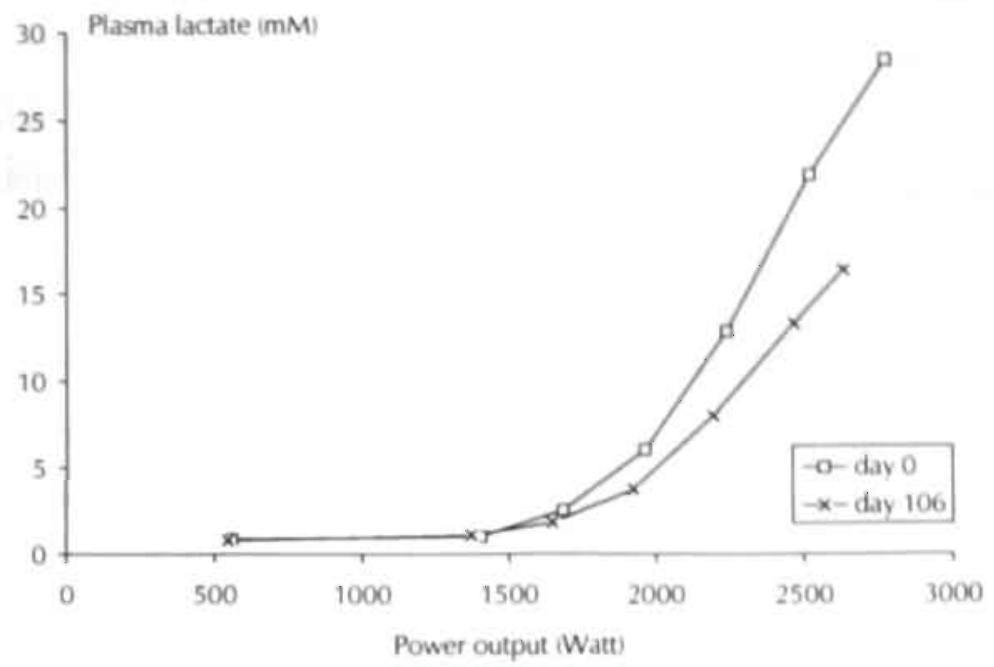

Fig. 8.6 Mean plasma lactate levels of the horses in group S3 at day 0 and day 106. The difference between test days proved to be significant (significant different regression parameters to fit the relationship).

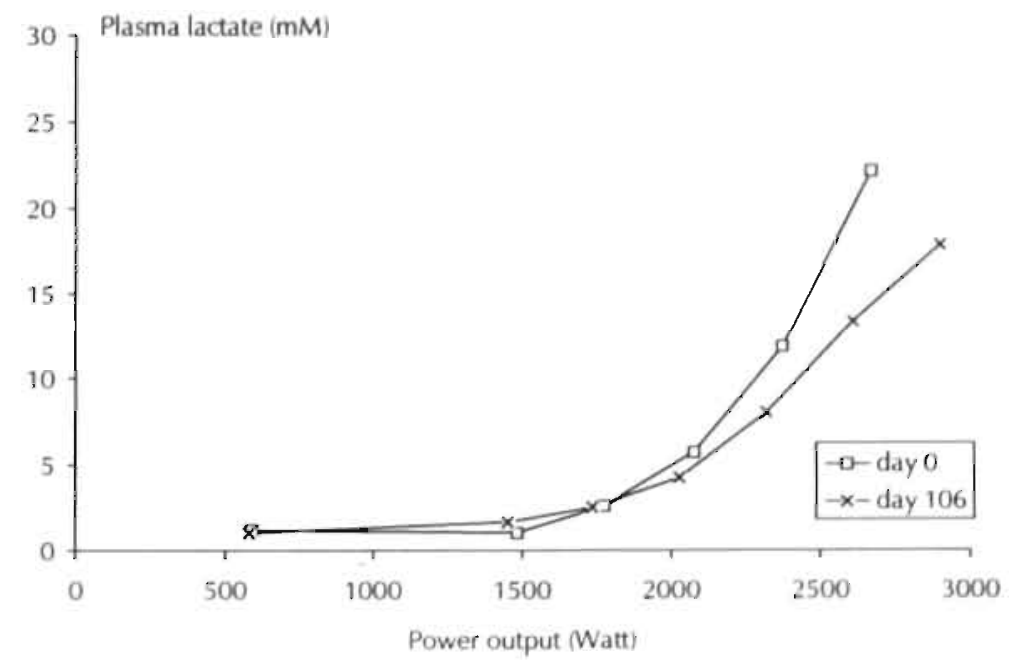

Fig. 8.7 Mean plasma lactate levels of the horses in group S6i at day 0 and day 106. The difference between test days proved to be significant isigrificant different regression parameters. to fit the relationship). 


\subsubsection{Biochemical parameters in muscle biopsies}

Analysis of the biochemical parameters in muscle biopsies showed no consistent and physiologically significant differences between the three exercise protocols. Therefore, the results were pooled (Table 8.5 and 8.6).

The tissue content of phosphocreatine showed a decline during the course of the training program (Table 8.5). The tissue content of ATP remained constant throughout the training period. The tissue level of both ADP and AMP showed a small, but statistically significant transient increase during the course of the training period (Table 8.5). No consistent pattern could be observed with respect to the tissue content of IMP during the training program. The content of glycogen in the gluteus medius muscle transiently increased during the training period with a peak on day 64 after the start of the exercise protocol (Table 8.5). Thereafter, tissue levels of glycogen were not significantly different from the values measured on day 0 . The tissue lactate concentration was found to vary significantly during the training period with the lowest level on day 64 (Table 8.5).

During the incremental exercise test the content of phosphocreatine declined (Table 8.6). Although significant differences in the magnitude of the decline were found between test days and between the different groups, no consistent, physiological meaningful pattern could be identified. The same holds for the decline in tissue ATP content during the test. Tissue ADP and AMP remained virtually unchanged during the exercise tests (Table 8.6). In contrast, the level of IMP in the gluteus medius muscle significantly increased during the exercise test (Table 8.6). There was a tendency for a more pronounced accumulation of IMP on days 42 and 64 as compared to earlier and later test days.

Muscle glycogen content significantly decreased during the incremental exercise test (Table 8.6). Significant differences in the magnitude of the decline were present both within one group (day to day differences) and between groups (data not shown). However, no consistent and meaningful pattern in these differences could be identified. 
The increase in tissue lactate during the incremental exercise test was present in all groups investigated (Table 8.6). The pooled data show a rather constant increase of tissue lactate levels throughout the whole experimental period (Table 8.6).

With respect to these metabolic parameters measured in biopsies of skeletal muscle, no significant differences in pattern between the three groups of investigation on corresponding test days could be observed (data not shown).

\subsection{DISCUSSION}

The major objective of the present study was to investigate the effect of three and six 1-min anaerobic interval bouts added to endurance running at $60 \%$ of peak heart rate on performance capacity in the horse. The exercise intensity was controlled by means of the heart rate registration (1), aiming at a level $>90 \%$ of the peak heart rate.

In general, the present study indicates that endurance training consisting of 26 min endurance exercise at $60 \%$ of the peak heart rate for 3 times a week, resulted in a significant improvement of physical performance capacity. The exercise intensity of $60 \%$ of the peak heart rate is approximately equivalent with an intensity of $40 \%$ of maximal oxygen uptake (5). Both total running time until exhaustion and total amount of vertical work delivered until exhaustion, measured during incremental exercise tests, increased from day 0 till day 64 of the training period by $4-11 \%$ and $7-16 \%$, respectively. This is in contrast with the general assumption that performance capacity can only be improved with an exercise intensity above $70 \% \mathrm{VO}_{2 \max }$ (9). This finding is of practical relevance because starting the training with a relatively low intensity does increase performance capacity in the one hand, and probably helps to prevent exercise injuries on the other. Adding three or six 1-min intensive exercise bouts to the endurance training protocol did not result in a further improvement of physical performance in the incremental exercise test during this period of time. 
Table 8.5 Tissue content of mean values of biochemical paramesers in m. glutacus medius ibropsies were taken jus pror to a inc rement al exertive lest on day indicaledf. The data are presented as mean values of pooled data of the three groups under investigation.

\begin{tabular}{|c|c|c|c|c|c|c|c|}
\hline & \multicolumn{7}{|c|}{ Day of the experimental period } \\
\hline & 0 & 21 & $42^{\prime}$ & 64 & 84 & 106 & sed \\
\hline Clycogen & $652^{4} \pm 95$ & $690^{26} \pm 175$ & $765^{1 x} \pm 156$ & $824^{C} \pm 125$ & $756^{i 3} \pm 173$ & $614^{4} \pm 118$ & 5.6 \\
\hline Lactate & $14.3^{x} \pm 3.2$ & $13.0^{2} \pm 4.5$ & $11.8^{a b} \pm 5.6$ & $9.5^{b} \pm 5.0$ & $12.4^{4} \pm 3.2$ & $12.9^{41} \pm 4.7$ & 1.6 \\
\hline IMP & $0.03 \pm 0.07$ & $0.10 \pm 0.16$ & $0.01 \pm 0.03$ & $0.11 \pm 0.10$ & $0.10 \pm 0.09$ & $0.03 \pm 0.05$ & 0.04 \\
\hline$P C$ & $93.3^{4} \pm 21.6$ & $90.7^{a} \pm 8.2$ & $85.7^{\mathrm{ab}} \pm 13.0$ & $69.6^{\mathrm{cd}} \pm 18.1$ & $60.8^{\mathrm{d}} \pm 12.8$ & $75.5^{1 x} \pm 13.6$ & 6.4 \\
\hline ATP & $23.6^{\mathrm{ab}} \pm 3.2$ & $22.6^{21} \pm 1.9$ & $24.1^{\mathrm{ab}} \pm 3.0$ & $25.1^{\mathrm{ab}} \pm 1.7$ & $25.2^{a b}+2.4$ & $25.8^{6}+2.4$ & 1.5 \\
\hline ADP' & $2.10^{2} \pm 0.39$ & $2.10^{\mathrm{a}} \pm 0.39$ & $2.29^{\mathrm{ab}} \pm 0.27$ & $2.55^{\mathrm{b}} \pm 0.18$ & $2.46^{\mathrm{b}} \pm 0.20$ & $2.09^{4} \pm 0.21$ & 0.14 \\
\hline AMP & $0.10^{\mathrm{a}} \pm 0.08$ & $0.12^{\mathrm{ab}} \pm 0.11$ & $0.16^{\mathrm{b}} \pm 0.12$ & $0.24^{c} \pm 0.08$ & $0.10^{\mathrm{a}} \pm 0.05$ & $0.07^{-1} \pm 0.03$ & 0.03 \\
\hline
\end{tabular}

The data are expressed as $\mu$ molig dry weight (mean $\pm s d)$ with the standard error of differences within groups $(n=12)$. Significunt difterences are indicated with superscripts a,b.c $(P<0.05)$. In case of identical superscript letters no difference was found: 
lable 8.6 Mean difference of tissue content of biochemical parameters in $\mathrm{m}$. glutaeus medius in biopsies taken jus prior to and immediately after an incremental exercise t-est on different days.

\begin{tabular}{|c|c|c|c|c|c|c|c|}
\hline & \multicolumn{7}{|c|}{ Day of the experimental period } \\
\hline & 1 & 21 & 42 & 64 & 84 & 106 & sed \\
\hline Glycogen & $229 \pm 166$ & $\overline{211 \pm 187}$ & $203 \pm 212$ & $329 \pm 199$ & $258+269$ & $201 \pm 176$ & 7.7 \\
\hline Laclate & $-60.4 \pm 32.4$ & $-74.8 \pm 43.2$ & $-74.7 \pm 42.7$ & $-85.5 \pm 32.4$ & $-79.0 \pm 40.1$ & $-70.2+28.4$ & 14.9 \\
\hline IMP & $-1.96^{\mathrm{ab}} \pm 1.02$ & $-2.43^{a b} \pm 1.90$ & $-2.87^{a} \pm 2.23$ & $-2.58^{2} \pm 1.56$ & $-1.29^{b} \pm 1.23$ & $-1.21^{10} \pm 1.42$ & 0.64 \\
\hline$P C$ & $61.3^{\mathrm{a}} \pm 32.2$ & $55.5^{a b} \pm 19.8$ & $37.4^{b} \pm 26.7$ & $46.0^{a b} \pm 18.8$ & $40.5^{b} \pm 12.0$ & $543^{\text {at }} \pm 13.2$ & 9.24 \\
\hline ATP & $8.8 \pm 6.7$ & $6.9 \pm 5.0$ & $5.5 \pm 5.2$ & $8.3 \pm 5.1$ & $6.4 \pm 4.5$ & $9.5 \pm 4.2$ & 2.3. \\
\hline ADP & $0.08 \pm 0.60$ & $0.09 \pm 0.50$ & $-0.14 \pm 0.42$ & $0.05 \pm 0.55$ & $0.12+0.41$ & $0.04+0.39$ & 0.21 \\
\hline $\mathrm{AMP}$ & $-0.06 \pm 0.11$ & $-0.06 \pm 0.11$ & $-0.08 \pm 0.17$ & $-0.06 \pm 0.08$ & $0.02+0.06$ & $0.01 \pm 0.05$ & 0.05 \\
\hline
\end{tabular}

The dat are presented as pooled values of the three groups under investigation and expressed as $\mu$ molig dry wright imean 2 ud wath ihe

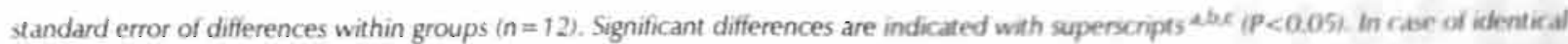
superscript letters no difference was present. 


\section{Chapter 8}

However, prolongation of the training protocal up to 106 days showed that physical performance assessed by the incremental exercise tests declined to pre-training levels in the groups without sprints and with three intensive exercise bouts. In the group with six intensive, anaerobic exercise bouts this decline in performance capacity at the end of the training period was not observed. Although no clear explanation for the decline in test performance can be given, it is possible that the stimulus consisting of zero or 3 sprints became physiologically less effective for maintaining the performance capacity. The data suggest that 6 bouts of intensive exercise were able to provide a sufficient stimulus to maintain performance capacity at the same level. It is suggested that exercise of low intensity elicits only some initial improvement in physical performance capacity, but for maintenance and/or a further development of the physical performance capacity a higher intensity and volume is necessary. It is of interest to note that Weltman et al. (15) observed a similar phenomenon in femaie athletes subjected to training at and above the lactate threshold.

One might argue that the decline in performance of the groups without and with 3 intensive exercise bouts was a sign of overtraining. However, this explanation is unlikely since this effect was absent in the group with 6 intensive exercise bouts, the group of horses with the highest training volume. In addition, other signs of overtraining, such as a lowered food intake, a decrease in body weight, irritability or stiffness, were not observed.

In all groups a lower plasma lactate level at a given power output was observed. This indicates that training with a predominant aerobic character, as in protocol $\mathrm{SO}$, is sufficient to improve the capacity of aerobic energy production in originally untrained. horses.

The gradual increase of plasma volume, as seen during the whole experimental period, is corroborating, the findings of Knight et al. (7). Mckeever et al. (10), however, found a faster increase of plasma volume in the first weeks of a training program. This may be explained by a lower training status of the horses involved in the study of McKeever et all. (10), which is supported by a lower plasma volume at the start of the 
experiment (16 litre in the study of McKeever et al. (10) vs. 23 litre and 22 litre in the study of Knight et al. (7) and the present study, respectively).

In contrast to plasma volume, red blood cell volume increased only in the first three weeks of the experiment in the groups without and with 6 intensive exercise bouts. These data suggest that regular low-intensity exercise is sufficient to elicit an increase in red blood cell volume. It is unclear to what extent changes in red cell volume are associated with changes in performance capacity.

The pattern of improvement of physical performance in incremental exercise tests was not associated with changes in the resting tissue content of the most relevant high-energy phosphates in $\mathrm{m}$. gluteus medius (phosphocreatine, ATP). Interestingly, glycogen levels showed a transient increase during the course of the training period in the three groups studied. It is unlikely that the alterations in the tissue carbohydrate store offers a feasible explanation for the differences in work capacity between the three groups of horses at the end of the training program.

Improvement of physical exercise during the incremental exercise test might be related to the specific changes in metabolic components in gluteus medius muscle that occur during the test The findings of this study strongly suggest that in horses, subjected to an intensive anaerobic training protocol, a high level of work output can be maintained without excessive loss of tissue high-energy phosphates and glycogen. This finding suggests that the metabolic energy conversion keeps pace with improvement of physical performance capacity at the end of the experimental period.

In conclusion, endurance training at $60 \%$ of the peak heart rate transiently increased performance capacity in an exercise test. Adding three or six 1-min exercise bouts at $>90 \%$ of the peak heart rate did not further increase exercise capacity. However, the data of the present study suggest that in order to maintain a higher level of fitness for several months three 1-min high-intensity exercise bouts per training are not sufficient. 


\section{Chapter 8}

\subsection{REFERENCES}

1. Asheim A., O. Knudsen, A. Lindholm, O. Rülcker, B. Saltin. Heart rates and blood lactate concentrations of standardbred horses during training and' racing. J Am Vet Med Assoc 157: 304-312, 1970.

2. Ástrand P.O., K. Rodahl. Textbook of Work Physiology. Physiological bases of exercise. McGraw-Hill Book Company, New York, 1986.

3. Bergström 1.. Percutaneous needle biopsy of skeletal muscle in physiological and clinical research. Scand I Clin Invest 35: 609-616, 1975.

4. Essen-Gustavsson B., D. McMiken, K. Karistrom, A. Lindholm, S.G.B. Persson, J. Thornton. Muscular adaptation of horses during intensive training and detraining. Eq Vet J 21: 27-33, 1989.

5. Evans D.L., R.J. Rose. Maximum oxygen uptake in racehorses: changes with training and prediction from submaximal cardiorespiratory measurements. In: Gillespie J.R., N.E. Robinson (eds): Equine Exercise Physiology 2 Edition: 52-67, Edwards Brothers, Ann Arbor, USA, 1987.

6. Genstat 5 Committee (R.W. Payne, chairman, P.W. Lane, secretary). Design and analysis of experiments. In: Genstat 5 Release 3 Reference Manual, Clarendon Press, Oxford, 1993, pp. 461-538.

7. Knight P.K., A.K. Sinha, R.J. Rose. Effects of training intensity on maximum oxygen uptake: In: Persson S.G.B., A. Lindholm, L.B. Jeffcott (eds): Equine Exercise Physiology 3: 77-82, Almgivist \& Wiksell Tryckeri, Uppsala, 1991.

8. Lindholm A., K. Piehl. Fibre composition, enzyme activity and concentrations of metabolites and electrolytes in muscles of standardbred horses. Acta Vet Scand 15: $287-$ $309,1974$.

9. McArdle W.D., F.I. Katch, V.L. Katch. Training for anaerobic and aerobic power. In: Exercise Physiology. Energy, nutrition and human performance: 347:370, Lea \& Febiger, Philadelphia, 1986.

10. McKeever K.H., W.A. Schurg, S.H. Jarrett, V.A. Convertino, Exercise training-induced hypervolemia in the horse. Med Sci Sports Exerc 19: 21-27, 1987.

11. Passoneau J.V.. Lactate. Fluorometric method. In: Bergmeyer H.U. (ed): Methods of 
Enzymatic Analysis, New York Academic Press, New York, 1974, pp. 1464-1472.

12. Saltin, B., P.D. Gollnick. Skeletal muscle adaptibility: Significance for metabolism and performance In: L.D. Peachy (ed.): Handbook of Physiology, Section 10. Skeletal muscle. Williams and Wilkins, Baltimore, 1983, pp. 555-631.

13. Van der Vusse G.J., W.A. Coumans, F.H. Van der Veen, A. Drake. ATP, creatine phosphate and glycogen content in human myocardial biopsies: markers for the efficacy of cardioprotection during aorta coronary bypass surgery. Vasc Surg 18: 127-134, 1984.

14. Van der Vusse G.J., G.M.E. Janssen, W.A. Coumans, H. Kuipers, R.J.M.M. Does, F. Ten Hoor. Effect of training and 15-, 25- and $42 \mathrm{~km}$ contests on the skeletal muscle content of adenine an guanine nucleotides, creatine phosphate and glycogen. Int J Sports Med 10: S146-S152, 1989.

15. Weltman A., R.L. Seip, D. Snead, J.Y. Weltman, E.M. Haskvitz, W.S. Evans, J.D. Veldhuis, A.D. Rogol. Exercise Training at and Above the Lactate Threshold in Previously Untrained Wornen. Int J Sports Med 13: 257-263, 1992.

16. Wijnants J., H. Van Belle. Single-run high performance liquid chromatography of nucleotides, nucleosides and major purine bases and its application to different tissue extracts. Anal Biochem 144: 258-266, 1985. 
Chapter 8 
Chapter 9

GENERAL DISCUSSION 


\subsection{GENERAL DISCUSSION}

The main purpose of the present study was to investigate in horses the effect of various training protocols and types of training on physical performance capacity. Horse races involve exercise bouts of a duration varying mostly between 1 and $10 \mathrm{~min}$. Such exercise intensities are equivalent to approximately 100-120\% $\mathrm{VO}_{2 \max }$ (McArdle et al, 1996). Therefore $\mathrm{VO}_{2 \max }$ as well as anaerobic power are important requirements for success in races. Consequently, horse training should involve endurance running to increase and maintain general aerobic endurance, and high intensity intermittent exercise to enhance $\mathrm{VO}_{2 \max }$ and anaerobic power.

In most of the studies presented in this thesis, in which different training protocols were compared, the number of animals in each experiment was rather limited. The reason for the relatively small number of animals in each group was a practical one. All the training and testing had to be done during normal working hours during daytime while only one treadmill and a limited time span was available. To overcome a possible drawback of the limited number of horses a randomized block design was chosen and rather conservative statistical techniques were used.

One of the key procedures used in the present series of studies was measuring physical performance capacity under standardized conditions. For this purpose, an incremental treadmill test until exhaustion was used in which each 2 minutes the workload was increased. One reason for using this test protocol was to be able to compare the data from this study with those from the Swedish research group who used a comparable protocol (14). Another reason for choosing stages of two minutes was that we wanted to obtain steady state conditions at each stage as far as heart rate and plasma lactate concentration were concerned. In contrast to our study, other authors have used steps of one minute in tests to obtain information about the relationship between heart rate and plasma lactate concentration $(4,6,8,16)$. Knight et al. (1991) used a mixed protocol with steps of 3 and 1 minute, while Galloux et al. (1995) applied 3 minute steps in studying lactate kinetics. Most cardiorespiratory variables will be close to steady state after 1 minute $(5,15)$. However, in humans as well as in horses plasma lactate levels will require 2-3. minute to reach a steady state (5, 12, 15). Therefore, two minute steps fulfilled the requirement that steady state conditions for heart rate and plasma lactate concentration should be obtained. 


\section{Chapter 9}

In the exercise test, as used in the present study, a $6.25 \%$ incline was used. This choice was made because using an incline will reduce the maximal running speeds and consequently the risk of self inflicted injuries at high speeds. For the same reason also in training exercise an incline of $6.25 \%$ or $10 \%$ was used. In addition, the application of an incline has the advantage that it enables one to calculate delivered vertical work and power output (3).

In the present study maximal running time (s) and total amount of work ( $k J)$ during the incremental treadmill test have been used as parameters for physica! performance capacity. This choice was made because no specific tests were available to test aerobic and anaerobic capacity separately. Although at present no better laboratory tests are available, it may be questioned in as much these parameters reliably and specifically reflect competitive physical performance capacity. It has been demonstrated in humans that the power output at $\mathrm{VO}_{2 \max }$ is a better indicator for endurance capacity than $\mathrm{VO}_{2 \max }$ itself (9). Therefore, as the exercise protocol was the same each time, the maximal running time is to be considered as a good indicator for endurance capacity. In humans it has been shown that a time trial test is a valid indicator for endurance capacity $(1,10)$. Also in horses, a practical approach would be to conduct time trials on the track lasting 3-6. min. However, a time trial on an outdoor track can hardly be standardized because of different and uncontrollable environmental and track conditions, while taking samples during the test is more complicated or even impossible compared to a test on a treadmill (15). Therefore we decided that an indoor treadmill test would be the best acceptable approximation to assess performance capacity and changes therein.

In a number of the separate studies from this thesis significant changes in physical performance capacity have been observed. In some other cases small and statistically insignificant changes were found. However, even under standardized conditions, a certain unexplained variability in physiological response to exercise has to be anticipated. Kuipers et al. (1985) showed in man, who were repeatedly tested on a cycle ergometer, that the magnitude of the variability in maximal aerobic performance was approximately $5 \%$. In addition, the magnitude of the variability may differ between individuals. This kind of variation has also to be taken into account in horses.

In most of the present studies an improvement of the performance capacity measured as total work-output was found. The data obtained do not allow to determine to what extent the improvement was caused by a change in aerobic or 
anaerobic capacity. One of the key parameters to be able to answer this question would have been direct measurement of the maximal oxygen uptake $\left(\mathrm{VO}_{2 \max }\right)$ and the maximally accumulated oxygen deficit $(2,13,15)$. Because of technical limitations these parameters could not be measured in the present experimental setup. However, the incremental treadmill test as used in the present study includes an aerobic as well as an anaerobic component.

For training practice proper training is important. Often heart rate is used as an indicator for exercise intensity. The present study confirmed that heart rate can be used as an indicator for the exercise intensity. As long as the target heart rate does not exceed 230 beats/minute, a "Sport tester" can be used for training purposes. This is usually applicable in endurance training and interval training at submaximal (lower than $100 \%$ of the maximal oxygen uptake) intensity. However, when higher maximal heart rates are to be expected, heart rate measurements should be done with other types of heart rate monitors. In practice it may be often the case that proper equipment for measuring maximal heart rates is lacking. The present study has shown (chapter 3) that it is possible to estimate the maximal heart rate by measuring heart rate and plasma lactate concentration at different submaximal speeds. By using an algorithm in which the input parameters are plasma lactate concentration and heart rate assessed during more than 5 submaximal workloads, the maximal heart rate can be estimated with an acceptable accuracy. In the present training studies certain percentages of the maximal heart rate (relative heart rate) were used (3). Since the algorithm for estimating maximal heart was applied in a retrospective way, no further tests have been conducted in the other experiments described in this thesis to investigate whether the measured peak heart rate was the maximal attainable heart rate under physiological conditions.

An important aim of the present study was to search for markers that are indicative of overtraining and staleness in an early stage (chapter 4). One of the interesting findings was that it remains difficult to diagnose overtraining in an early stage. In spite of the large number of variables measured and of the frequency of the measurements, the first signs of overtraining syndrome were not of physiologicall origin but of behavioral nature, i.e., reluctance to exercise, increased irritability, and lower food intake. None of the measured blood chemical variables could be identified as a sensitive and early marker of overreaching and staleness. The same holds for biochemical parameters measured in biopsies of the exercising leg muscle. None of the 


\section{Chapter 9}

alterations in the tissue content of high-energy phosphates and related compounds and of glycogen could be used as a reliable indicator of imminent overreaching and staleness. The consequence of this observation is that at present horse trainers have to rely more on behavioral characteristics than on laboratory measurements as support for prevention of overreaching and staleness. In addition, the horses were not able to perform the exercise work bouts. However, this observation in the laboratory situation is of limited value for practical horse training, because of the difficulty to use standardized training protocols in the field. Another significant finding was that overtraining was not seen as long as the intensive training sessions were alternated with light exercise at the following day (chapter 4). For sport practice this emphasizes the importance of alternating high intensity and low intensity training bouts.

Other studies in this thesis have been devoted to investigate the effect of different combinations of endurance training and interval training. For this purpose the effect of the number of repetitions of the high intensity exercise (chapter 8 ) as well as the intensity of the interval bouts was studied (chapter 6, chapter 7) was studied.

It was shown that various combinations of endurance and high intensity exercise have a positive effect on performance capacity. Although endurance training is an important basic component in the training program, the results of the present study demonstrate that high-intensity exercise has to be performed in order to further increase performance capacity. Considering the results of the various studies in which different combinations of endurance running plus intermittent high intensity exercise bouts were used, the conclusions can be drawn that high intensity aerobic exercise of 3-4 times three minutes at an intensity of $\sim 90 \%$ of the peak heart rate and anaerobic exercise 6 times 1 -minute at an intensity above the $\mathrm{VO}_{2 \max }$ is necessary to exploit performance ability to its full capacity. The results of this study indicate that high intensity aerobic exercise ( $\sim 90 \%$ of the peak heart rate) is preferred above exercise of lower intensities. It is assumed that this is caused by inducing changes of the red cell volume and on the energy converting pathways in the muscle that are involved in the exercise. In practice it is always advocated that high intensity exercise should be preceded by a period of low intensity endurance training. Although such a training protocol has not been experimentally investigated in full detail, it should be mentioned that also in the present study a period of endurance running preceded the period with 
exercise at a higher level (i.e., a combination of aerobic and anaerobic exercise; chapter 5). However, this procedure is still rather based on tradition than on experimental data.

Since performance capacity did change by various combinations of endurance plus intermittent high intensity exercise, this must have a physiological basis. The increase in performance could partly be explained by increases in red cell volume. Muscle biopsies were taken to look for possible biochemical adaptations to the various training protocols. In the present study muscle glycogen and energy-rich phosphates were determined. Other indices such as buffer capacity and some key enzymes like citrate synthase were not determined because of practical limitations. In general, skeletal muscle glycogen levels in the rested muscle increased during the course of the different training protocols applied. In this respect, no physiologically significant differences could be observed between the training protocols. A common observation was the decline in tissue ATP, phosphocreatine and glycogen during the maximal exercise test, indicating that skeletal muscle energy conversion is seriously challenged during the performance tests. The observation that, in general, the type of training did not alter the extent of these changes in high-energy phosphates and glycogen suggests that skeletal muscle energy metabolism adequately adapts to the increased demand of energy during the course of the experimental protocal as in most protocols physical performance showed a significant improvement. In cases that no physical improvement could be detected, it cannot be excluded that an incomplete adaptation of muscle energy metabolism is (partly) responsible for the observed lack of enhanced exercise performance (chapters 5-8).

A remarkable and intriguing finding of the present study is that periormance capacity in the horses used in the present study could be maintained at an elevated level for several months by a light training intensity of $60 \%$ of the peak heart rate (chapter 7). This is of practical relevance because trainers are generally reluctant to lower the training frequency of the training intensity because they are afraid of performance loss. The present study indicates that this assumption may not hold. It is possible that this does not hold for maintaining maximal performance capacity, but only to keep moderately increased periormance capacity above a basal untrained level. The present study also showed that intensive training programs could be conducted without the occurrence of overuse injuries as is often encountered in race horse training. It is 
Chapter 9

conceivable that this was attributed to the lower running speed by the inclination of the treadmill, to the carefully designed training protocol with gradual increases in training intensity and volume and to the standardized warming-up procedure.

Several findings of the present study have relevance for practical horse training, such as:

- Heart rate can be used as an indicator for exercise intensity.

- When only submaximal heart rates can be measured, maximal heart rate can accurately be estimated using an algorithm with submaximal plasma lactate concentrations and submaximal heart rates.

-At present, no sensitive laboratory variables are available to detect overtraining in an early stage. Early markers are of behavioral origin.

- Overtraining can be avoided by alternating intensive training bouts with light exercise.

- For increasing performance capacity high intensity intermittent exercise at $100 \%$ $\mathrm{VO}_{2 \max }$ or above has to be conducted.

- After a training period light intensity exercise is sufficient to maintain performance capacity at a relatively high level.

- When a training program is built up gradually injuries can be avoided.

\subsection{REMAINING QUESTIONS FOR FUTURE RESEARCH}

Although several training protocols in which interval and endurance training. have been combined were described in the present thesis, still relatively little is known about the optimal combination of endurance and high intensity exercise. More well-controlled studies are required to provide conclusive answers to these questions. If practically possible, increased numbers of animals in each group might improve the statistical power of the studies.

Since it is still debatable in how far treadmill testing reflects performance capacity under field conditions, a question which has to be addressed is to which extent the results obtained with an incremental exercise test on a treadmill reflect the achievement during competitive performance or field tests. At present such studies are hard to conduct. If, however, indoor tracks would become available in which weather and surface conditions can be controlled an answer to this question can be provided.

An issue which is highly relevant for practical horse training is prevention and detection of early overtraining (overreaching) and advanced overtraining (overtraining 
syndrome or staleness). The present study failed to provide tissue biochemical and blood chemical markers for overreaching and staleness. Since the first signs of overtraining symptoms were of behavioral origin, future research should also focus on behavioral characteristics and features during the training process whereas other physiological and biochemical parameters should be explored. The actual mechanism of the effect of the increase of exercise intensity on the days with low-intensity endurance exercise in the overtraining study in evoking the overtraining syndrome should be subject of further study.

Compared to the changes seen in the overtraining study, the improvement of the performance capacity found in the aerobic and anaerobic exercise protocols in chapters 6-8 was limited. The used biochemical markers and total plasma volume changes as well as tissue high-energy phosphates and related compounds failed to provide insight into the mechanisms underlying changes in performance capacity. Since the assembled data suggest that a combination of aerobic and anaerobic exercise is necessary for reaching maximal performance capacity, further research should be done to identify the best combination of the two training components. To provide further insight into the contribution of aerobic and anaerobic energy production and training effects thereupon, the use of non-invasive tracers for metabolic alterations is recommended. The same holds for the mechanism(s) responsible for the possible changes in energy metabolism in the exercising horse and its skeletal muscles. With respect to elucidating underlying mechanisms also modern molecular biological techniques might be of help to gain more insight in the effect of training on the physical performance capacity of the horse.

Although high-intensity exercise seerns necessary for maximal training effects it is still unknown how many repetitions yield optimal results. This is relevant: because it has been shown that on the one hand high intensity exercise should be included in the training program, while on the other hand high volumes of high intensity exercise may increase the risk of staleness and injuries. It is likely that a certain training volume has to be attained, while it is to be expected that increasing the training volume will lead to overtraining. Therefore, it is also important to know the lowest level of volume and intensity for an individual horse which is sufficient to attain and sustain the maximal performance capacity. 


\section{Chapter 9}

\subsection{REFERENCES}

1. Coyle E.F., M.E. Teltner, S.A. Karetz, M.T. Hamilton. Physiological and biomechanica! factors associated with elite endurance cycling performance. Med Sci Sports Exerc 23: 93$107,1991$.

2. Eaton M.D., R.J. Rose, D.L. Evans, D.R. Hodgson. The assessment of anaerobic capacity of thoroughbred horses using maximal accumulated oxygen deficit. Aust Eq Vet 10: 86-91, 1992.

3. Evans D.L., R.J. Rose. Maximal oxygen consumption in racehorses. Changes with training state and prediction from submaximal indices of cardiorespiratory function. In: Gillespie J.R., N.E. Robinson (eds): Equine Exercise Physiology 2 Edition, Edwards Brothers, Ann Arbor, USA, 1987, PP. 52-67.

4. Evans. D.L., R.J. Rose. Cardiovascular and respiratory reponses to submaximal exercise training in the thoroughbred horse. Pfügers Arch 411: 316-321, 1988.

5. Evans D.L. The Cardiovascular System: Anatomy, Physiology, and Adaptations to Exercise and Training. In: Hodgson D.R., R.J. Rose (eds): The Athletic Horse, W.B. Saunders Company, Philadelphia, USA, 1994, pp. 129-144.

6. Evans D.L., J.E. Rainger, D.R. Hodgson, M.D. Eaton, RJ. Rose. The effects of intensity and duration of training on blood lactate concentrations during and after exercise. Eq Vet J Suppl 19: 422-425, 1995.

7. Galloux P., E. Barrey, B. Auvinet, J.P. Valette, R. Wolters. Kinematics of blood lactate concentration during an incremental treadmill test in saddle horses. Eq Vet J Suppl 18: 435-438, 1995.

8. Hanzawa K., K. Kubo, M. Kai, A. Hiraga, S. Watanabe. Effects of exercise on erythrocytes in normal and splenectomised Thoroughbred horses. Eq Vet J Suppl 19: 439-442, 1995.

9. Jeukendrup A.E., M.K.C. Hesselink, A.C. Snijder, H. Kuipers, H.A. Keizer. Physiological changes in male competitive cyclists after two weeks of intensified training. Int J Sports Med 13: 534-541, 1992.

10. Jeukendrup A.E., W.H.M. Saris, F. Brouns, A.D.M. Kester. A new validated endurance performance test. Med Sci Sports Exerc 28: 266-270, 1996.

11. Knight P.K., A.K. Sinha, R.J. Rose. Effects of training intensity on maximum oxygen uptake. In: Persson S.G.B., A. Lindholm, L.B. Jeffcott (eds): Equine Exercise Physiology 3, Almqvist \& Wiksell Tryckeri, Uppsala, 1991, pp. 77-82.

12. Kuipers H., F. Verstappen, H. Keizer, P. Geurten, and G. van Kranenburg. Variability of aerobic performance in the laboratory and its physiological correlates. Int I Sports Med 6: $197-201,1985$. 
13. Medbo J.I., A.C. Mohn, I. Tabata, R. Bahr, O. Vaage, O.M. Sejersted. Anaerobic capacity determined by maximal accumulated O2 deficit. J Appl Physiol 64: 50-60, 1988.

14. Persson S.C.B. Evaluation of exercise tolerance and fitness in the performance horse. In: Snow D.H., S.G.B. Persson, R.J. Rose (eds): Equine Exercise Physiology, Burlington Press, Cambridge, 1983, pp. 441-457.

15. Rose R.J., D.R. Hodgson. Clinical Exercise testing. In: Hodgson D.R., R.J. Rose (eds): The Athletic Horse, W.B. Saunders Company, Philadelphia, USA, 1994, pp. 245-257.

16. Rose R.J., C.M. King, D.L. Evans, C.M. Tyler, D.R. Hodgson. Indices of exercise capacity in horses presented for poor racing performance. Eq Vet I Suppl 19: 418-421, 1995. 
Chapter 9 
SAMENVATTING 
In dit proefschrift worden effecten van verschillende vormen van training op het prestatievermogen van paarden beschreven. De paarden werden op stal gehouden en getraind op een tredmolen en aan een stapmolen. De trainingsprogramma's waren afgestemd op de capaciteiten van elk paard afzonderlijk. Om veranderingen in het prestatievermogen te kunnen beoordelen werden de paarden elke 2-4 weken onderworpen aan een fitness test. Door onderzoek van spierbiopten en bloedmonsters, en door het registreren van de hartfrequentie is getracht een aantal belangrijke onderdelen van de inspanningsfysiologie van het paard te beschrijven.

Enkele algemene aspecten van de inspanningsfysiologie bij mens en paard worden beschreven in de hoofdstukken $\mathbf{1}$ en 2 . Het uithoudingsvermogen (duurvermogen) wordt in hoofdzaak bepaald door de maximale hoeveelheid zuurstof die kan worden gebruikt in het lichaam. Dit is vooral afhankelijk van bloedsomloop en ademhaling. Paarden zijn in vergelijking met de mens heel bijzondere atleten. De hartfrequentie kan bij het paard een veel hogere waarde bereiken dan bij de mens het geval is. Bij paarden zijn maximale waarden gevonden van 240-260 slagen per minuut, humaan ligt de bovengrens tussen 200 en 210 slagen per minuut. Door bij inspanning een grote hoeveelheid rode bloedlichaampjes, in rust opgeslagen in de milt, te gaan gebruiken, waardoor het aantal circulerende rode bloedcellen met $50 \%$ kan toenemen, wordt de zuurstof transport capaciteit van het bloed sterk vergroot. In vergelijking met rust kan de maximale zuurstofopname bij paarden 40-50 keer groter worden. Een topsportpaard kan maximaal ongeveer tweemaal zoveel zuurstof per kg lichaamsgewicht transporteren dan een menselijke topatleet.

Door training wordt geprobeerd het prestatievermogen te verbeteren. Hierbij wordt gebruik gemaakt van de eigenschap van het lichaam om zich aan te passen aan de opgelegde belasting. Bij elke trainingssessie wordt het bestaande evenwicht in de belaste cellen verstoord. Gedurende de herstelfase na de training kan dit evenwicht niet 
Samenvatting

alleen weer worden hersteld, maar kan er ook een vergroting van de belastbaarheid optreden (overcompensatie). Als omvang en intensiteit van de trainingsarbeid geleidelijk toenemen zal ook het prestatievermogen toenemen totdat het maximaal haalbare is bereikt. De aard van de training moet in het algemeen zo specifiek mogelijk worden afgestemd op het doel. Voor een kortdurende intensieve prestatie moeten korte maximale sprints worden uitgevoerd, voor een prestatie die uren moet worden volgehouden is duurtraining met een lagere intensiteit nodig. Bij prestaties van paarden van ongeveer 1 tot 10 minuten is de maximale zuurstofopname capaciteit van groot belang, terwijl tevens het sprintvermogen maximaal ontwikkeld moet zijn. Voor een maximaal prestatievermogen is dan ook een intensief trainingsprogramma nodig, waarbij omvang en intensiteit zorgvuldig moeten worden afgestemd op de belastbaarheid van de weefsels van elk paard. Trainingsarbeid beneden het optimale niveau leidt niet tot het maximale prestatievermogen, terwijl een te intensief programma overtraining of blessures tot gevolg kan hebben. In hoofdstuk 2 is ingegaan op de fysiologie van de skeletspier en op de stofwisseling. De veranderingen die optreden onder invloed van training zijn kort weergegeven.

Eén van de doelen van het in dit proefschrift beschreven onderzoek was om overtraining vroegtijdig te kunnen diagnostiseren en zo ernstiger beschadigingen te voorkomen. Daarnaast is getracht de effecten van verschillen in ormvang en intensiteit in trainingsprogramma's voor paarden te beschrijven om in de: praktijk een zo effectief mogelijke training met een minimale kans op blessures mogelijk te maken.

Voor het beoordelen van de histochemie en de biochemie van een spier is met behulp van een hoile naald een klein stukje weefsel uit die spier gehaald. Het is dan de yraag of zo'n klein stukje weefsel wel een goede weergave van de eigenschappen van de gehele spier kan opleveren. Derhalve is in hoofdstuk $\mathbf{3}$ gekeken naar de herhaalbaarheid van de bepalingen in spierbiopten door op 3 verschillende plaatsen op twee verschillende dieptes telkens drie monsters te nemen. Hieruit kwam naar voren 
dat de verschillen bij oppervlakkig genomen monsters groter waren dan bij dieper genomen monsters. Voor biochemisch onderzoek moet de voorkeur worden gegeven aan een plaats circa $17 \mathrm{~cm}$ schuin naar beneden en naar achteren ten opzichte van het heupbeen van het paard. Voor histochemisch onderzoek was het een plek $10 \mathrm{~cm}$ horizontaal achter het heupbeen gelegen. Door het belang dat is gehecht aan de biochemische analyses is gekozen voor de voor dit onderzoek gevonden optimale plaats.

De intensiteit van de trainingsarbeid kan worden beoordeeld aan de hand van de hartfrequentie uitgedrukt als percentage van de maximaie hartfrequentie van elk paard. In de praktijk kan de hartslag relatief eenvoudig worden vastgelegd met behulp van een hartslagmeter. De maximale hartslag is echter zo hoog dat die veelal niet kan worden gemeten met een hartslagmeter. in hoofdstuk $\mathbf{4}$ is de betrouwbaarheid van een hartslagmeter onderzocht en is getracht de maximale hartslag van een paard te schatten op basis van de hartslag en de plasma-lactaat concentratie bij submaximale inspanning. Het bleek dat bij frequenties lager dan 231 slagen per minuut de hartfrequentie betrouwbaar gemeten kan worden met een hartslagmeter, terwijl bovendien de maximale hartirequentie kan worden geschat op basis van gemeten submaximale waarden en de daarbij behorende plasma-lactaat spiegels.

In de loop van een intensief trainings- en/of wedstrijdprogramma kan overtraining optreden. De paarden zijn dan lusteloos, lopen stijf, eten slecht en vermageren. De geplande trainingsarbeid kan veelal niet meer worden volbrächt, terwijl ook eventuele wedstrijdprestaties tegenvallen. In hoofdstuk $\mathbf{5}$ is getracht door een intensief trainingsschema overtraining op te wekken en dit dan vrorgtijdig te diagnostiseren met behulp van spier- en bloedmonsters en van een zorgvuldige klinische diagnose. Het bleek pas mogelijk om overtraining op te wekken vanaf het moment dat de intensiteit van de arbeid op de dagen tussen de intensieve training 
Samenvatting

werd opgevoerd van circa 140 slagen per minuut naar circa 180 slagen per minuut. Het bloedonderzoek leverde geen aanwijzing op voor een vroegtijdige diagnose. Gedragsveranderingen en het afnemen van de eetlust waren de belangrijkste symptomen van overtraining. De paarden lieten wel een verbetering van het uithoudingsvermogen zien van $40-50 \%$ (gemeten als hoeveelheid werk tot uitputting).

In hoofdstuk 6 worden de effecten op het uithoudingsvermogen beschreven van duurtraining met een verschillende duur en intensiteit. In alle groepen werd een verbetering van het uithoudingsvermogen gezien van circa $10 \%$. In de groepen met een hogere intensiteit van de trainingsarbeid werden de grootste effecten in het bloed gezien in de vorm van een stijging van de hoeveelheid rode bloedcellen per $\mathrm{kg}$ lichaamsgewicht. Een beperkte trainingsduur van 6 weken was al voldoende om deze veranderingen te realiseren.

Met decelfde paarden als gebruikt in houfdotuh 6 is getracht de effecten van een combinatie van duurtraining en sprinttraining op het uithoudingsvermogen te bestuderen. In de periode tussen beide experimenten ( 2 maanden) werden de paarden licht getraind om de effecten van de vorige trainingsperiode te laten verdwijnen. Desondanks bleek het uithoudingsvermogen in twee maanden niet te zijn verminderd (hoofdstuk 7). Bovendien kon gedurende het verloop van de trainingsperiode geen verandering van het uithoudingsvermogen worden vastgesteld.

In hoofdstuk 8 zijn de effecten van sprinttraining met 0,3 of 6 sprints van 1 minuut beschreven. Alle paarden lieten gedurende de trainingsperiode een verbetering van het uithoudingsvermogen zien vergelijkbaar in grootte met de verbetering door duurarbeid (hoofdstuk 6). Naar het einde van de trainingsperiode bleek echter dat alleen de groep met 6 sprints het verworven niveau op peil wist te houden.

In hoofdstuk 9 worden de resultaten zoals beschreven in dit proefschrift samengevat en in een breder kader geplaatst. 
CURRICULUM VITAE 
De auteur van dit proefschrift is geboren op 7 januari 1944 te Vledder. Nadat in 1964 aan het J.H. Tromp-Meesters Lyceum te Steenwijk het Gymnasium- $\beta$ diploma werd behaald, begon hij in hetzelfde jaar aan de Rijksuniversiteit Utrecht met de studie diergeneeskunde. In 1971 legde hij het dierenartsexamen met goed gevolg af. Dat examen werd evenals de propaedeuse behaald met het predikaat "met genoegen". Van september 1971 tot november 1973 was hij werkzaam als keuringsdierenarts / bacterioloog te Den Haag, van november 1973 tot maart 1983 was hij praktizerend dierenarts te Coevorden. Van maart 1983 tot heden volgde een dienstverband als onderzoeker bij het "Praktijkonderzoek Rundvee, Schapen en Paarden" te Lelystad, alwaar de werkzaamheden zoals beschreven in dit proefschrift zijn uitgevoerd. 
DANKWOORD 
Dit proefschrift is tot stand gekomen dankzij de medewerking en het enthousiasme van een groot aantal mensen. Ik wil iedereen bedanken die hieraan, direct of indirect, een bijdrage heeft geleverd. Toch wil ik een aantal mensen graag met name noemen.

Allereerst wil ik mijn ouders en schoonouders bedanken voor de steun die ik tijdens mijn studie en daarna mocht ontvangen. Zij hebben me altijd gestimuleerd in het oppakken van nieuwe dingen ook al betekende dat meer inzet van hun kant.

Joke, Hielke en Bas, jullie hebben me altijd de gelegenheid gegeven mijn werk ook thuis voort te zetten. Dit is vaak ten koste gegaan van de tijd die voor jullie beschikbaar was. Hoewel de overgang van de praktijk te Coevorden naar het onderzoek te Lelystad door velen niet is begrepen, is dit door jullie nooit ter discussie gesteld. We hebben met elkaar steeds geprobeerd de dingen te doen die we leuk vonden. Of ik van de koek niet een onevenredig groot stuk heb geconsumeerd, vraag ik me met regelmaat af. Zonder jullie support was dit proefschrift nooit tot stand gekomen.

Durk Minkema, jouw stimulerende optreden tijdens mijn studietijd en je bereidheid tot discussiëren over onderzoek daarna heeft mijn interesse voor research gewekt.

Jules Smit, Jan Obermeyer, Tjitse Smeding, jullie zijn voor mij "de mannen van het eerste uur" met een niet te onderschatten inbreng bij het tot stand komen van deze studie. Jullie benadering van het trainen van paarden, met veel aandacht voor de noden van het individuele dier, heeft geleid tot een praktische toetsing van het in dit proefschrift beschreven onderzoek.

Rinus de Jong, jouw steun en vertrouwen bij mijn werk binnen het praktijkonderzoek heb ik als zeer motiverend ervaren. Je opmerking "Dat geloof je toch zelf niet" staat in mijn geheugen gegrift.

Wim Vrijsen en Jan Loman, jullie hebben al die jaren de padrden verzorgd en gatraind en meegewerkt aan de fitness tests. Vele dagen hebben jullie doorgebracht op de denderende tredmolen om de trainingsprogramma's uit te voeren. Dank zij jullie nauwkeurige wijze van werken en jullie inzet, ook ıp zon- en feestdagen als het voor het onderzoek nodig was, is dit proefschrift mogelijk geworden. 
Prof. Huisman, Arend Schot, Elly Nicaise, jullie medewerking bij het vastleggen van de ecg's en het maken van de Evan's blue oplossingen was onontbeerlijk voor dit onderzoek.

Tony Bruggink, jouw inbreng heeft geresulteerd in een opzet van het praktijkonderzoek waardoor deze studie mogelijk werd. Onze discussies zijn voor mij van grote betekenis geweest bij de uitvoering van het hier beschreven onderzoek.

Gidi, vanaf het eerste uur heb ik met jou mogen samenwerken. Jouw normbesef, je kritische en praktische instelling, je kennis, je tomeloze inzet en je onvoorwaardelijke steun, zowel binnen als buiten de werksituatie waardeer ik bijzonder.

Geert, steeds weer was je bereid om de geheimen van de statistiek in een voor mij passend tempo te onthullen. Je actieve participatie in de opzet van het onderzoek en de verwerking van de gegevens en jouw zoektocht naar een verantwoorde benadering van de dataset betekenen een grote meerwaarde voor het onderzoek.

Een woord van dank ook voor de leden van de beoordelingscommissie, Prof. Dr. ir. W.H.M. Saris, Prof. Dr. A. Huson, Dr. H.A. Keizer, Prof. Dr. R.J. Rose en Dr. F.T.J. Verstappen voor de zorgvuldige beoordeling.

Tenslotte, mijn promotores, Prof. Dr. H. Kuipers en Prof. Dr. G.J. van der Vusse:

Harm, jouw nooit aflatende en stimulerende benadering en je snelheid van werken bij het steeds maar weer corrigeren van de manuscripten hebben me er doorheen gesleept.

Ger, jouw kritische blik heeft bijzonder verhelderend gewerkt. De kwaliteit waarmee jij de manuscripten nakeek is nauwelijks te evenaren.

Harm en Ger, ik heb het als een voorrecht ervaren om onder jullie leiding dit proefschrift te mogen voltooien. 
